\title{
Examining the Effects of Ligand Variation on the Electronic Structure of Uranium Bis(imido) Species
}

John J. Kiernicki, ${ }^{\dagger}$ Maryline G. Ferrier, ${ }^{\ddagger}$ Juan S. Lezama Pacheco, ${ }^{\circ}$ Henry S. La Pierre, ${ }^{\ddagger}$ Benjamin W. Stein, ${ }^{\ddagger}$ Matthias Zeller,,$\S$ Stosh A. Kozimor, ${ }^{\ddagger}$ and Suzanne C. Bart*†

†H.C. Brown Laboratory, Department of Chemistry, Purdue University, West Lafayette, Indiana 47907, United States ₹ Los Alamos National Laboratory, Los Alamos, New Mexico 87545, United States

${ }^{\partial}$ Stanford University, Stanford, California 94305, United States

$\S$ Department of Chemistry, Youngstown State University, Youngstown, Ohio 44555, United States 


\section{Table of Contents:}

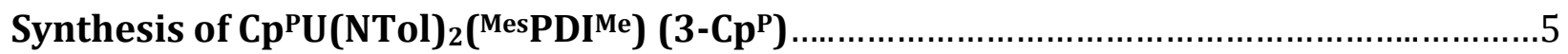

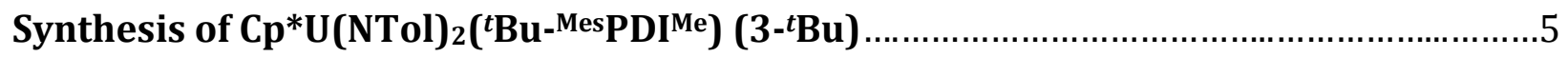

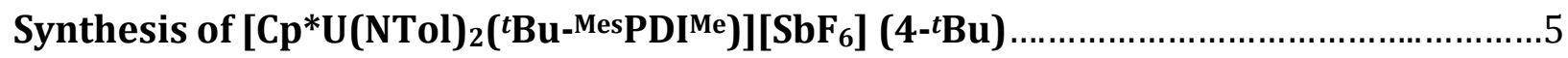

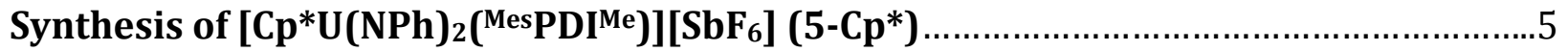

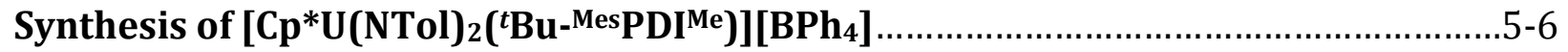

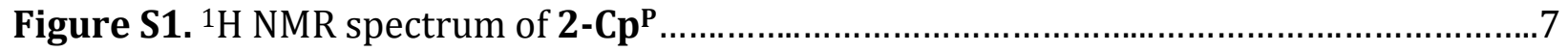

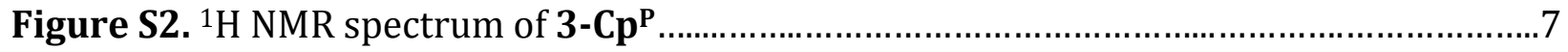

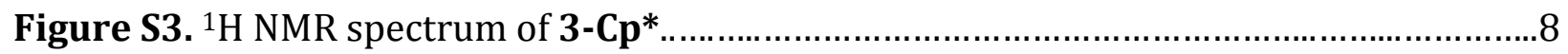

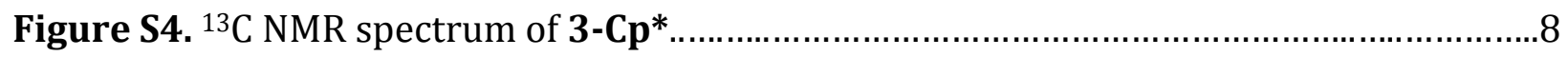

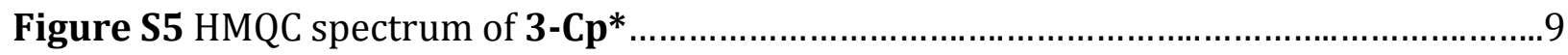

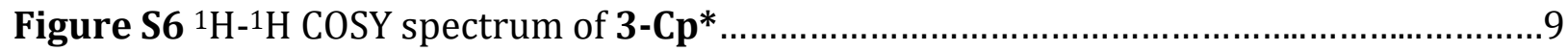

Figure S7 ${ }^{13} \mathrm{C}$ and DEPT-135 spectra of 3-Cp* ..............................................................

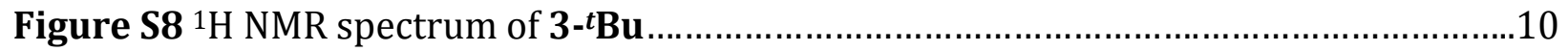

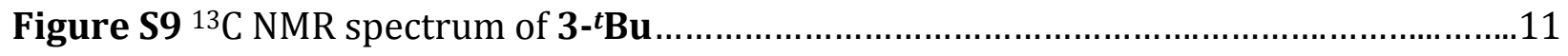

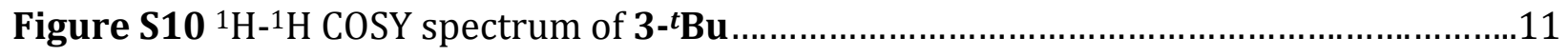

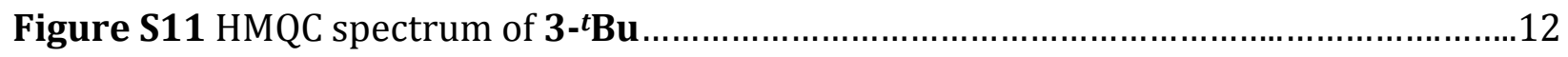

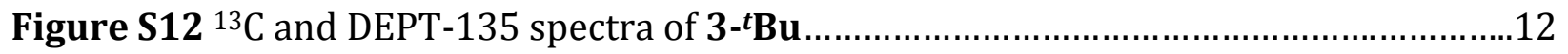

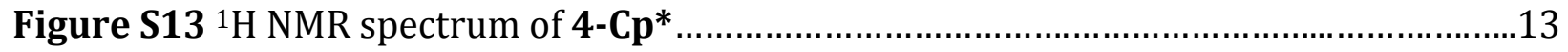

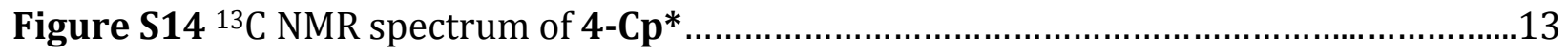

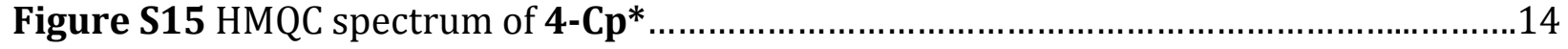

Figure S16 Infrared spectrum of 4-Cp* .......................................................................14

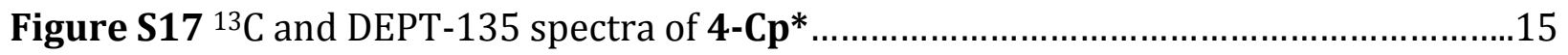

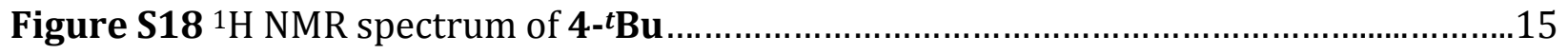

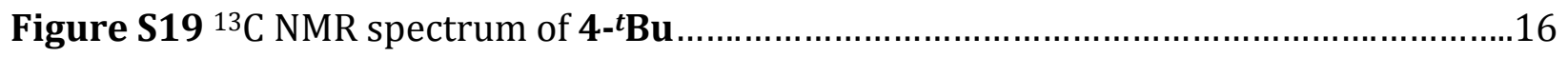

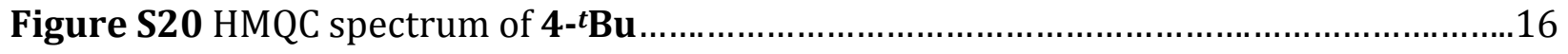

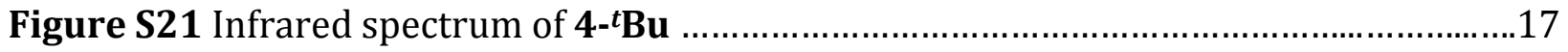




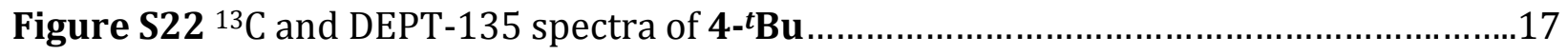

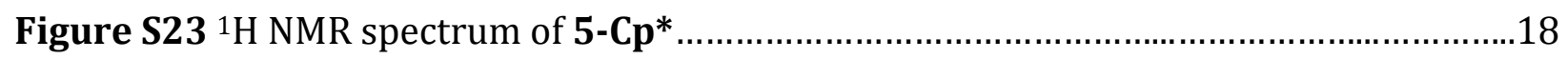

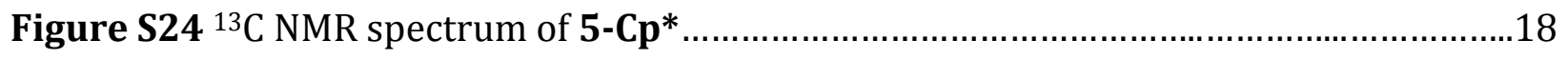

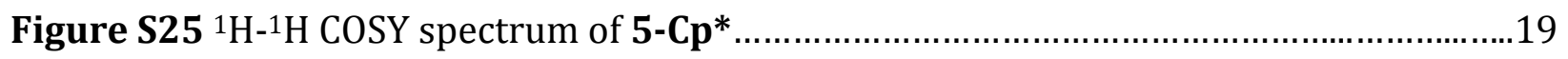

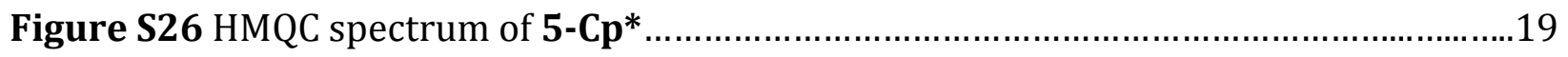

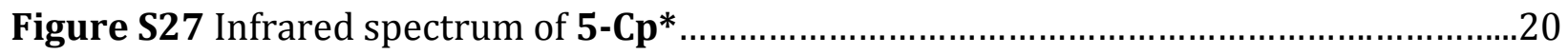

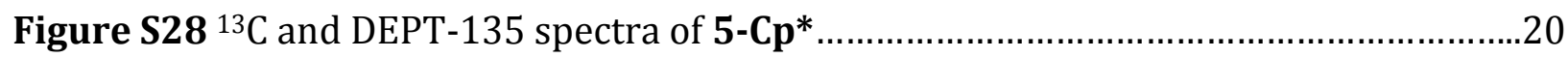

Figure S29 ${ }^{1} \mathrm{H}$ NMR spectra of 3-U(VI)-Cp* and 4-Cp* with residual solvent......................21

Figure S30 Electrochemical plots of $\mathrm{Ph}-\mathrm{N}=\mathrm{N}-\mathrm{Ph}$ and Tol-N=N-Tol..................................21

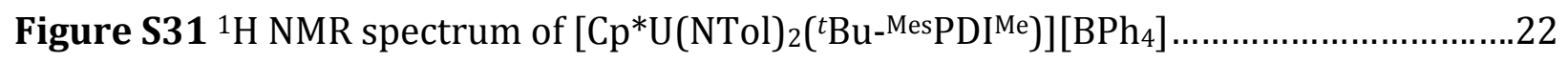

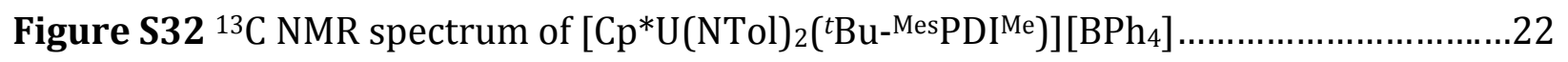

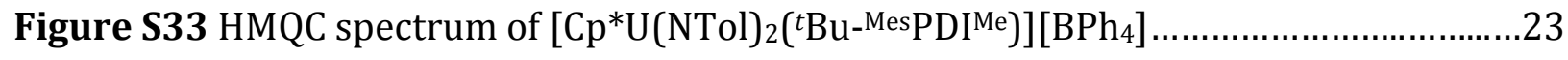

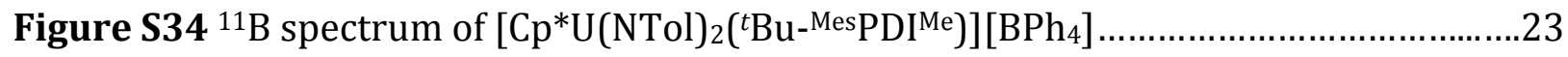

Figure S35 ${ }^{13} \mathrm{C}$ and DEPT-135 spectra of $\left[\mathrm{Cp} * \mathrm{U}\left(\mathrm{NTol}_{2}\left({ }^{t} \mathrm{Bu}^{-\mathrm{MesPDI}}{ }^{\mathrm{Me}}\right)\right]\left[\mathrm{BPh}_{4}\right] \ldots \ldots \ldots \ldots \ldots \ldots . . . . .24\right.$

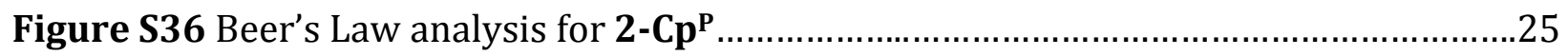

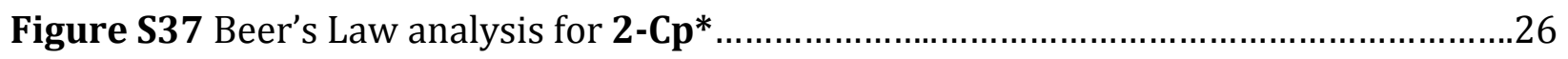

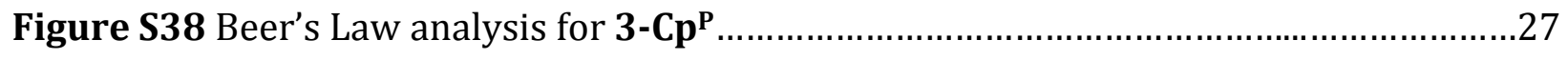

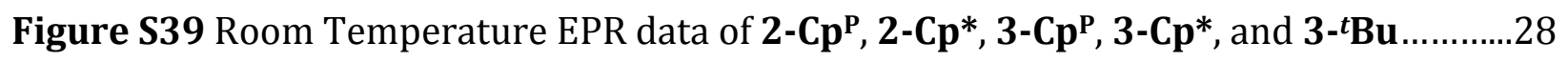

Figure S40 Low Temperature EPR data of 2-CpP, 2-Cp*, 3-CpP, 3-Cp*, and 3-tBu..............29

Figure S41 ${ }^{1} \mathrm{H}$ NMR spectrum of the oxidation of 3-Cp* in a sealed J. Young NMR tube......30

Figure $\mathbf{S 4 2}{ }^{1} \mathrm{H}$ NMR spectrum of the oxidation of $\mathbf{3}^{-}{ }^{t} \mathbf{B u}$ in a sealed J. Young NMR tube........31

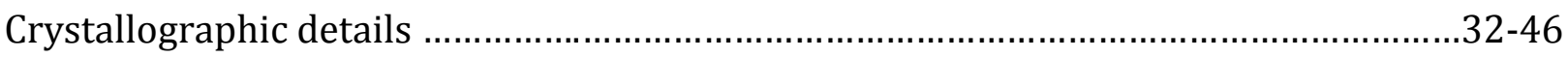

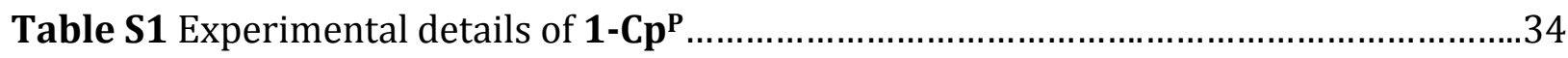

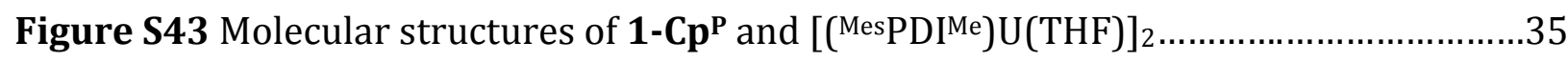

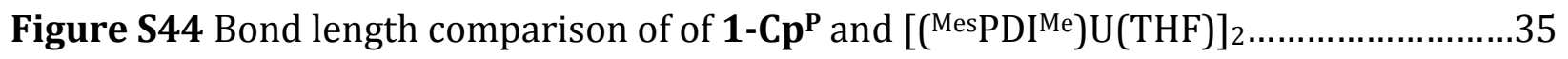

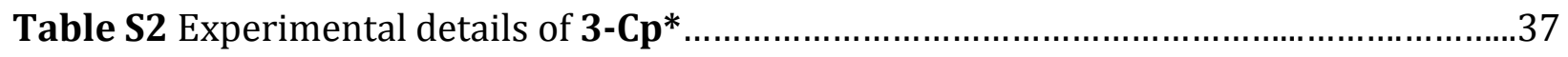

Table S3 Experimental details of 3-tBu 
Table S4 Experimental details of 4-Cp* ........................................................................

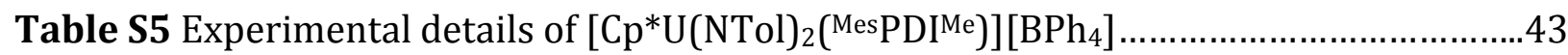

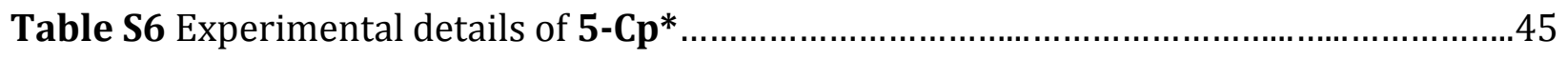

Experimental details of XAFS data collection..................................................................... 
Synthesis of $\mathrm{Cp}^{\mathrm{P}} \mathrm{U}(\mathrm{NTol})_{2}$ (MesPDIMe) (3-Cp $^{\mathrm{P}}$ ). A $20 \mathrm{~mL}$ scintillation vial was charged with $0.250 \mathrm{~g}(0.305 \mathrm{mmol})$ of $\left[\mathrm{Cp}{ }^{\mathrm{P} U}\left(\mathrm{MesPDI}^{\mathrm{Me}}\right)\right]_{2}$ and $5 \mathrm{~mL}$ of toluene. While stirring, $0.081 \mathrm{~g}$ $(0.608 \mathrm{mmol})$ of $p$-tolylazide was added resulting in the liberation of $\mathrm{N}_{2}(\mathrm{~g})$. After $15 \mathrm{~min}$, volatiles were removed in vacuo. The resulting solid was washed with cold pentane ($\left.35{ }^{\circ} \mathrm{C}\right)$ to afford dark brown solid $(0.259 \mathrm{~g}, 0.252 \mathrm{mmol}, 82 \%)$ assigned as $\mathrm{Cp}^{\mathrm{P} U}(\mathrm{NTol})_{2}\left(\mathrm{Mes}^{\mathrm{PDI}}{ }^{\mathrm{Me}}\right)$.

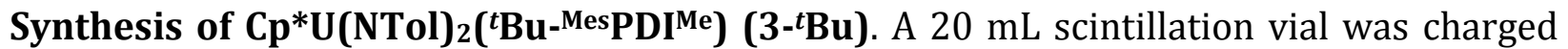
with $\mathrm{Cp}^{*} \mathrm{U}\left({ }^{\mathrm{t}} \mathrm{Bu}-\mathrm{Mes} P \mathrm{PI}^{\mathrm{Me}}\right)(\mathrm{THF})(0.200 \mathrm{~g}, 0.222 \mathrm{mmol})$ and $5 \mathrm{~mL}$ of toluene. While stirring, Tol-N=N-Tol (0.047 g, $0.224 \mathrm{mmol})$ was added resulting in an immediate darkening of the solution. After $5 \mathrm{~min}$, volatiles were removed in vacuo. The product was extracted into $n$ pentane, filtered over Celite, and dried. Recrystallization from concentrated $n$-pentane afforded brown powder $(0.130 \mathrm{~g}, 0.125 \mathrm{mmol}, 56 \%)$ assigned as $\mathrm{Cp} * \mathrm{U}(\mathrm{NTol})_{2}\left({ }^{t} \mathrm{Bu}-\right.$ MesPDIMe). Single, X-ray quality crystals were obtained from a concentrated $n$-pentane solution at $-35^{\circ} \mathrm{C}$.

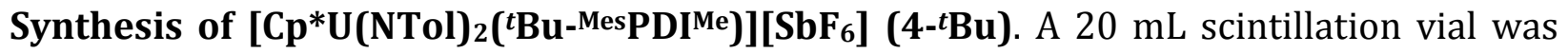
charged with $\mathrm{Cp}^{*} \mathrm{U}(\mathrm{NTol})_{2}\left({ }^{(} \mathrm{Bu}-\mathrm{Mes} \mathrm{PDI}^{\mathrm{Me}}\right)(0.183 \mathrm{~g}, 0.176 \mathrm{mmol})$ and $10 \mathrm{~mL} \mathrm{THF}$. While stirring, $\mathrm{AgSbF}_{6}$ was added and stirred for $2 \mathrm{hr}$. The solution was filtered over Celite and volatiles were removed in vacuo. The resulting solid was washed with $n$-pentane until the washings were clear and dried to yield brown powder $(0.210 \mathrm{~g}, 0.165 \mathrm{mmol}, 94 \%)$

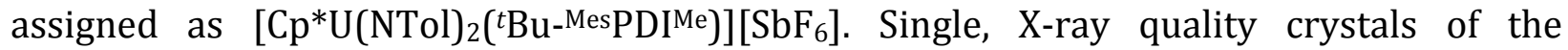
analogous tetraphenylborate complex precipitated from a concentrated THF/hexamethyldisiloxane (3:1) solution stored at room temperature.

Synthesis of $\left[\mathrm{Cp}^{*} \mathrm{U}(\mathrm{NPh})_{2}\left(\mathrm{MesPDI}^{\mathrm{Me}}\right)\right]\left[\mathrm{SbF}_{6}\right]$ (5-Cp*). A $20 \mathrm{~mL}$ scintillation vial was charged with $\left.\mathrm{Cp}^{*} \mathrm{U}(\mathrm{NPh})_{2}{ }_{2}{ }^{\mathrm{Mes} P D I}{ }^{\mathrm{Me}}\right)\left(2-\mathbf{C p}^{*}\right)(0.190 \mathrm{~g}, 0.199 \mathrm{mmol})$ and $5 \mathrm{~mL}$ THF. While stirring, $\mathrm{AgSbF}_{6}$ was added resulting in immediate deposition of silver metal on the walls of the vial. After $2 \mathrm{hr}$ of stirring, the solution was filtered over Celite and volatiles were removed in vacuo. The resulting solid was washed with n-pentane until the washings were clear and dried to yield red-brown powder $(0.210 \mathrm{~g}, 0.177 \mathrm{mmol}, 89 \%)$ assigned as $\left[\mathrm{Cp}^{*} \mathrm{U}(\mathrm{NPh})_{2}\left(\mathrm{Mes}^{\mathrm{PDI}}{ }^{\mathrm{Me}}\right)\right]\left[\mathrm{SbF}_{6}\right]\left(\mathbf{5}-\mathrm{Cp}^{*}\right)$. Single, X-ray quality crystals were obtained by slow

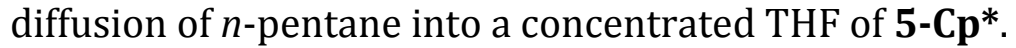

Synthesis of [Cp*U(NTol $)_{2}\left({ }^{t} \mathrm{Bu}^{*}\right.$-MesPDIMe $\left.)\right]\left[\mathrm{BPh}_{4}\right]$ A $20 \mathrm{~mL}$ scintillation vial was charged with $\mathrm{Cp}^{*} \mathrm{U}\left({ }^{t} \mathrm{Bu}-{ }^{-}{ }^{\mathrm{Mes}} \mathrm{PDI}^{\mathrm{Me}}\right)(\mathrm{THF})\left(\mathbf{1}^{-} \mathrm{Bu}\right)(0.140 \mathrm{~g}, 0.156 \mathrm{mmol})$ and $5 \mathrm{~mL}$ toluene. While stirring, Tol-N=N-Tol $(0.033 \mathrm{~g}, 0.157 \mathrm{mmol})$ was added resulting in an immediate darkening of the solution. After $5 \mathrm{~min}$, volatiles were removed in vacuo. The resulting solid was redissolved in $5 \mathrm{~mL} \mathrm{THF}$ and $\mathrm{AgBPh}_{4}(0.066 \mathrm{~g}, 0.155 \mathrm{mmol})$ was added and stirred for $3 \mathrm{hr}$. The solution was then filtered over Celite and dried. The crude solid was washed with $n$-pentane until the washing ran clear and dried to afford red-brown solid $(0.207 \mathrm{~g}, 0.153$ mmol, 98\%) assigned as [Cp*U(NTol $\left.)_{2}\left({ }^{t} \mathrm{Bu}-{ }^{M e s} \mathrm{PDI}^{\mathrm{Me}}\right)\right]\left[\mathrm{BPh}_{4}\right] .{ }^{1} \mathrm{H}$ NMR $\left(\mathrm{CDCl}_{3}, 500 \mathrm{MHz}\right.$, $\left.25^{\circ} \mathrm{C}\right) \delta=1.71\left(\mathrm{~s}, 9 \mathrm{H}, \mathrm{C}\left(\mathrm{CH}_{3}\right)_{3}\right), 1.77\left(\mathrm{~s}, 12 \mathrm{H}, o-\mathrm{CH}_{3}\right), 2.18\left(\mathrm{~s}, 6 \mathrm{H}, \mathrm{CH}_{3}\right), 2.57\left(\mathrm{~s}, 6 \mathrm{H}, \mathrm{CH}_{3}\right), 3.54$ (s, 6H, $p$ tol- $\mathrm{CH}_{3}$ ), 4.85 (d, $J=7.5,4 \mathrm{H}, p$ tol- $\left.\mathrm{CH}\right), 5.36$ (s, 15H, Cp*), 6.97 (t, $J=7.0,4 \mathrm{H}, \mathrm{BPh}_{4}-p$ $\mathrm{CH}$ ), 7.11 (s, $4 \mathrm{H}, m-\mathrm{CH}$ ), $7.14\left(\mathrm{t}, J=7.0,8 \mathrm{H}, \mathrm{BPh}_{4}-m-\mathrm{CH}\right), 7.42$ (d, $J=8.0,4 \mathrm{H}, p$ tol-CH), 7.52 $(\mathrm{m}, 8 \mathrm{H}, \mathrm{BPh} 4-\mathrm{o}-\mathrm{CH}), 8.62(\mathrm{~s}, 2 \mathrm{H}, m-\mathrm{pyr}-\mathrm{CH}) \cdot{ }^{13} \mathrm{C} \mathrm{NMR}\left(\mathrm{CDCl}_{3}, 126 \mathrm{MHz}, 25^{\circ} \mathrm{C}\right) \delta=7.34\left(\mathrm{Cp}^{*}-\right.$ $\left.\mathrm{CH}_{3}\right), 18.24$ (ptol- $\left.\mathrm{CH}_{3}\right), 18.71\left(o-\mathrm{Ar}-\mathrm{CH}_{3}\right), 20.22\left(\mathrm{CH}_{3}\right), 20.87\left(\mathrm{CH}_{3}\right), 30.61\left(\mathrm{C}\left(\mathrm{CH}_{3}\right)_{3}\right), 36.55$ 
$\left(C\left(\mathrm{CH}_{3}\right)_{3}\right), 121.70\left(\mathrm{BPh}_{4}-p-\mathrm{CH}\right), 123.52$ (ptol- $\left.\mathrm{CH}\right), 125.64,127.97(m-$ pyr- $\mathrm{CH}), 129.96(\mathrm{~m}-\mathrm{Ar}-$ $\mathrm{CH}), 130.36\left(\mathrm{BPh}_{4}-m-\mathrm{CH}\right), 136.46\left(\mathrm{BPh}_{4}-\mathrm{o}-\mathrm{CH}\right), 137.98,138.92(p$ tol- $\mathrm{CH}), 139.10,141.09$, 142.65, $155.87(C-\mathrm{N}=(\mathrm{Me})(\mathrm{Pyr})), 157.23\left(\mathrm{~N}=\mathrm{C}-C_{\mathrm{pyr}}\right), 164.49\left(\mathrm{BPh}_{4}\right.$-ipso-CH), 168.18 (p-pyr$\mathrm{CH}), 176.13\left(\mathrm{~N}=\mathrm{CCH}_{3}\right) .{ }^{11} \mathrm{~B} \mathrm{NMR}\left(\mathrm{CDCl}_{3}, 161 \mathrm{MHz}, 25^{\circ} \mathrm{C}\right) \delta=-6.33$. 


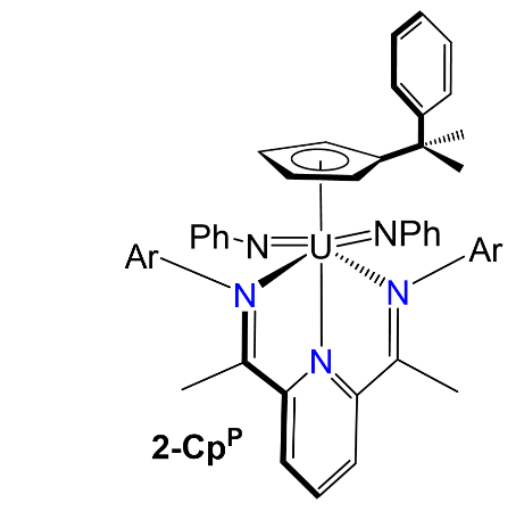

$\begin{array}{lllllllllllllllllllllllllllllllllllllllllll}40 & 38 & 36 & 34 & 32 & 30 & 28 & 26 & 24 & 22 & 20 & 18 & 16 & 14 & 12 & 10 & 8 & 6 & 4 & 2 & 0 & -2 & -4 & -6 & -8 & -10 & -12 & -14 & -16 & -18 & -20\end{array}$

Figure $\mathrm{S} 1{ }^{1} \mathrm{H}$ NMR spectrum $\left(\mathrm{C}_{6} \mathrm{D}_{6}, 300 \mathrm{MHz}, 25^{\circ} \mathrm{C}\right)$ of $\mathbf{2}-\mathbf{C p}^{\mathbf{P}}$

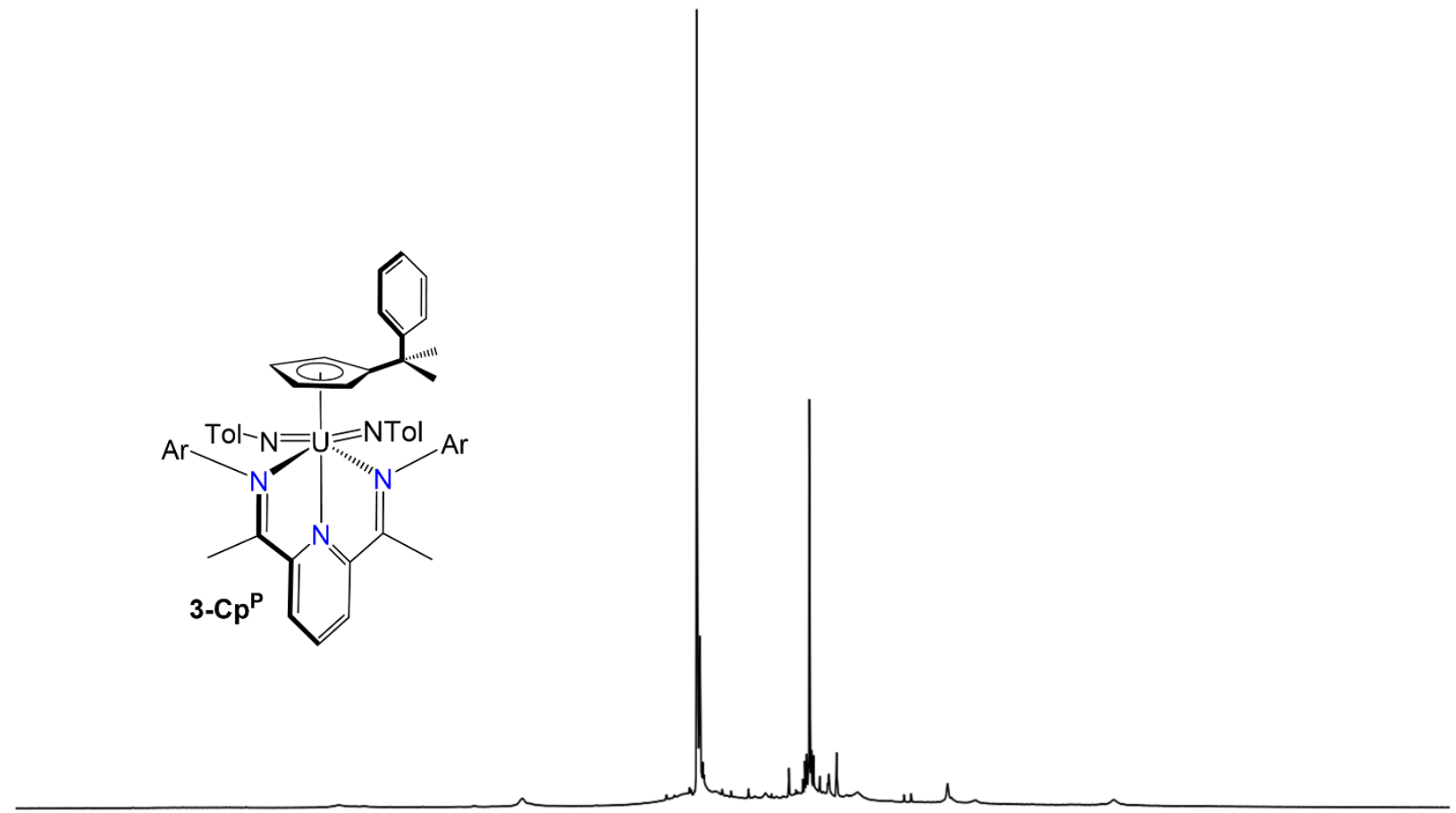

$\begin{array}{lllllllllllllllllllllllllllllllll}36 & 34 & 32 & 30 & 28 & 26 & 24 & 22 & 20 & 18 & 16 & 14 & 12 & 10 & 8 & 6 & 4 & 2 & 0 & -2 & -4 & -6 & -8 & -10 & -12 & -14 & -16 & -18 & -20 & -22 & -24 & -26\end{array}$

Figure S2 ${ }^{1} \mathrm{H}$ NMR spectrum $\left(\mathrm{C}_{6} \mathrm{D}_{6}, 300 \mathrm{MHz}, 25^{\circ} \mathrm{C}\right)$ of $\mathbf{3}-\mathrm{Cp}^{\mathbf{P}}$ 

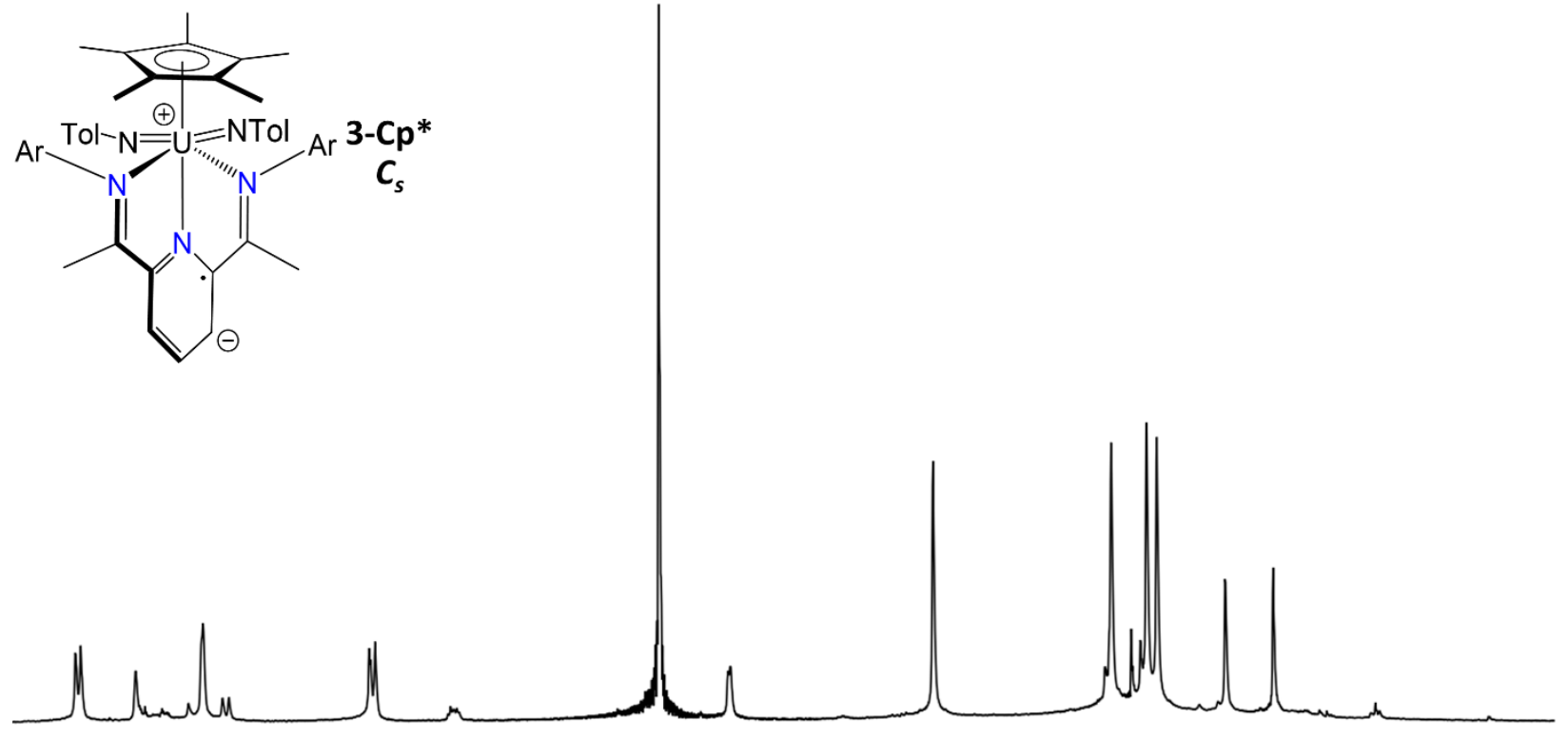

\begin{tabular}{|c|c|c|c|c|c|c|c|c|c|c|c|c|c|c|}
\hline & 7.0 & 6.5 & 6.0 & 5.5 & 5.0 & 4.5 & 4.0 & 3.5 & 3.0 & 2.5 & 2.0 & 1.5 & 1.0 & 0.5 \\
\hline
\end{tabular}

Figure S3 ${ }^{1} \mathrm{H}$ NMR spectrum $\left(\mathrm{C}_{6} \mathrm{D}_{6}, 300 \mathrm{MHz}, 25{ }^{\circ} \mathrm{C}\right)$ of $\mathbf{3}-\mathbf{C p}^{*}$

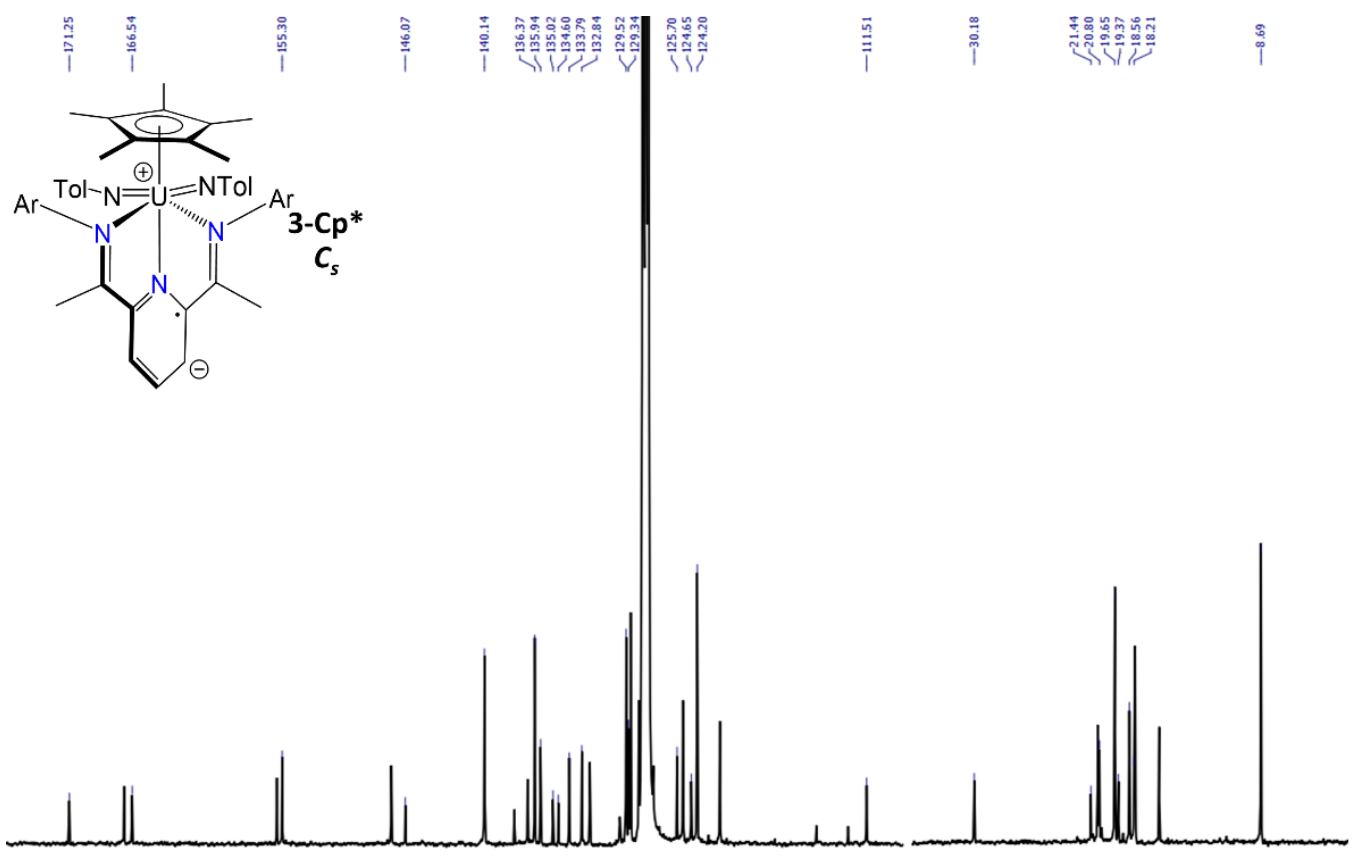

$\begin{array}{llllllllllllllllllll}75 & 170 & 165 & 160 & 155 & 150 & 145 & 140 & 135 & 130 & 125 & 120 & 115 & 110 & 30 & 25 & 20 & 15 & 10 & 5\end{array}$

Figure $\mathrm{S} 4{ }^{13} \mathrm{C}$ NMR spectrum $\left(\mathrm{C}_{6} \mathrm{D}_{6}, 126 \mathrm{MHz}, 25^{\circ} \mathrm{C}\right)$ of $\mathbf{3}-\mathbf{C p}{ }^{*}$ 


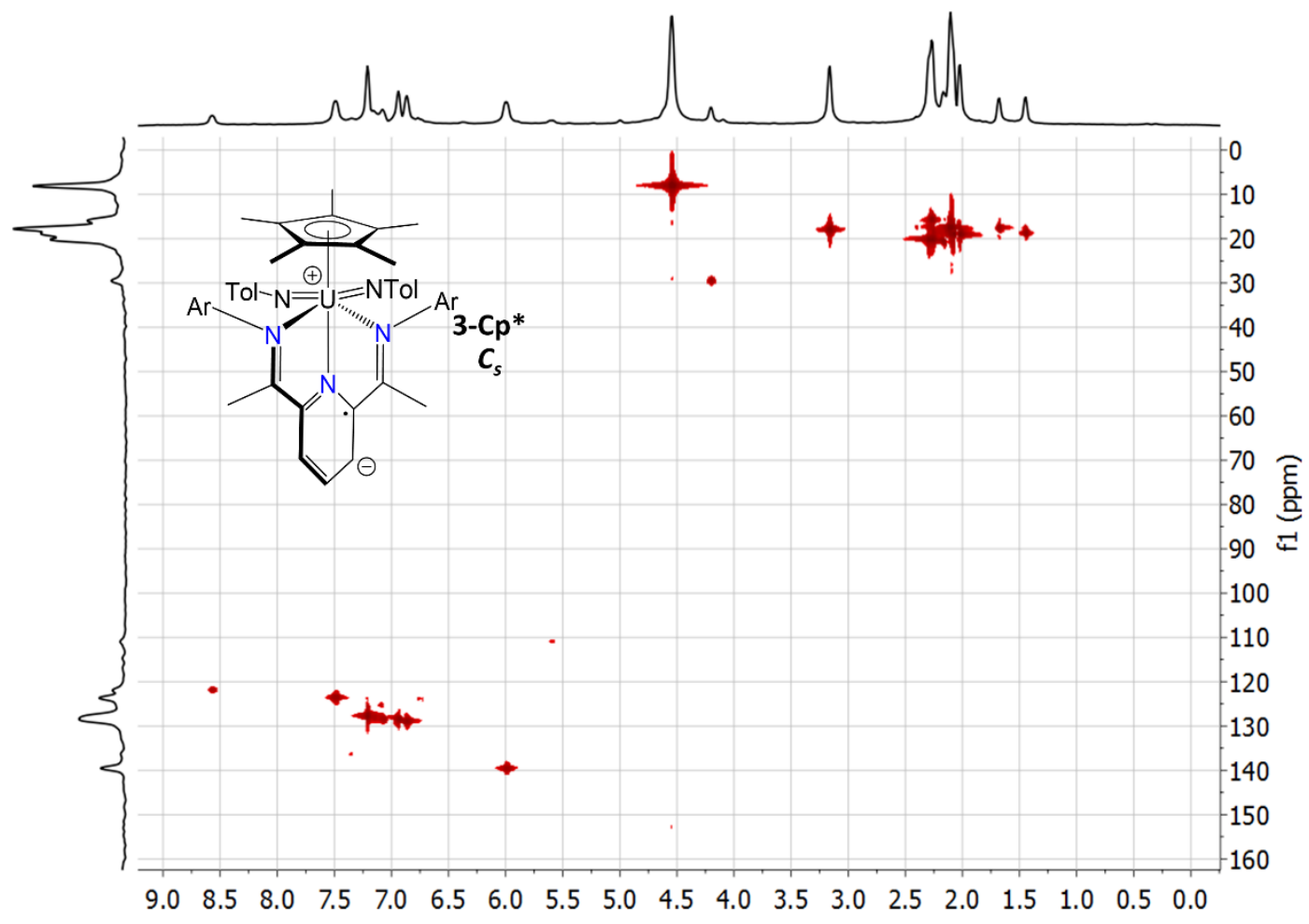

Figure S5 HMQC spectrum $\left(\mathrm{C}_{6} \mathrm{D}_{6}, 126 \& 500 \mathrm{MHz}, 25^{\circ} \mathrm{C}\right)$ of $\mathbf{3}-\mathrm{Cp}^{*}$

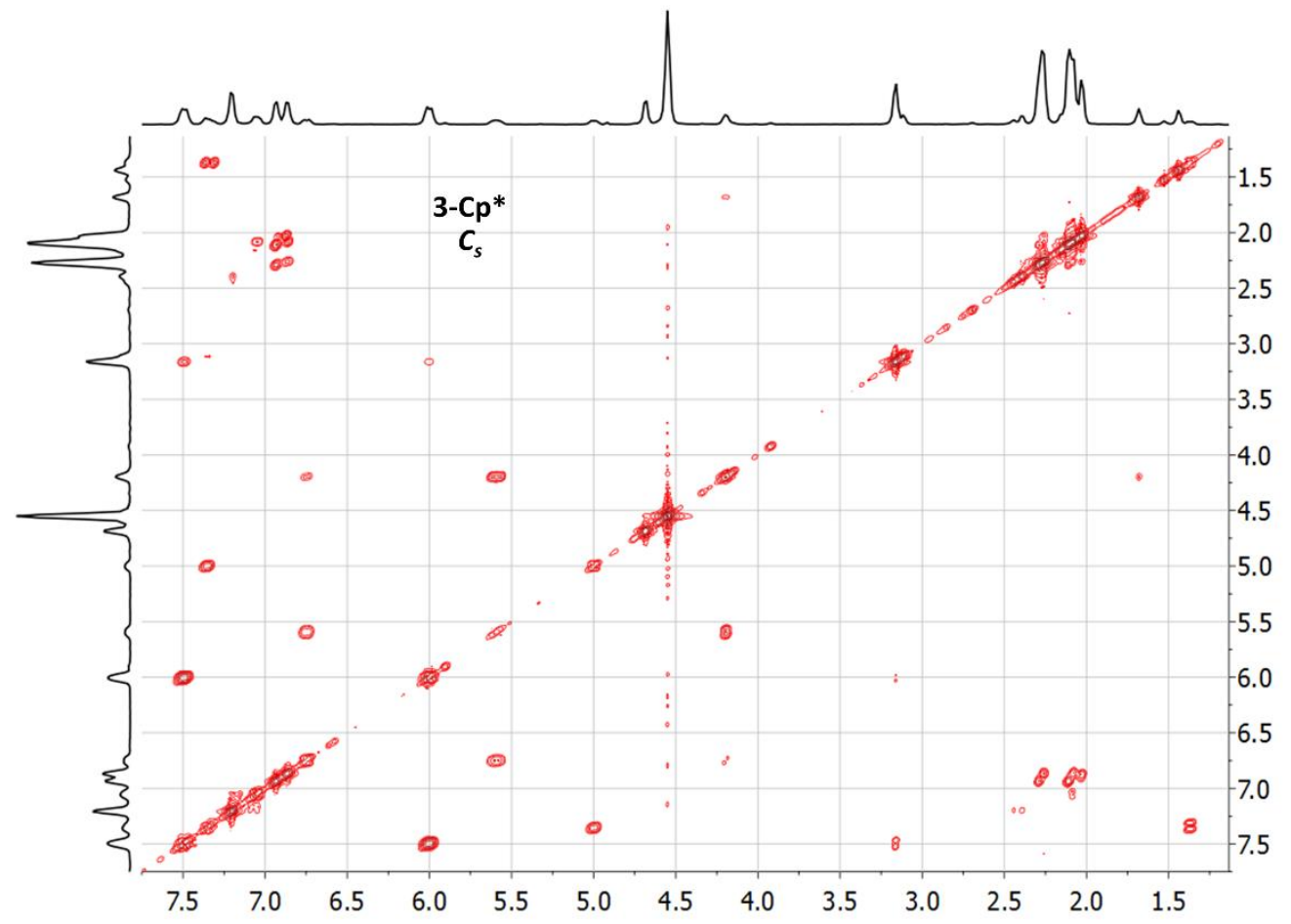

Figure S6 ${ }^{1} \mathrm{H}-{ }^{1} \mathrm{H}$ COSY spectrum $\left(\mathrm{C}_{6} \mathrm{D}_{6}, 500 \mathrm{MHz}, 25^{\circ} \mathrm{C}\right)$ of $\mathbf{3}-\mathbf{C p} *$ 


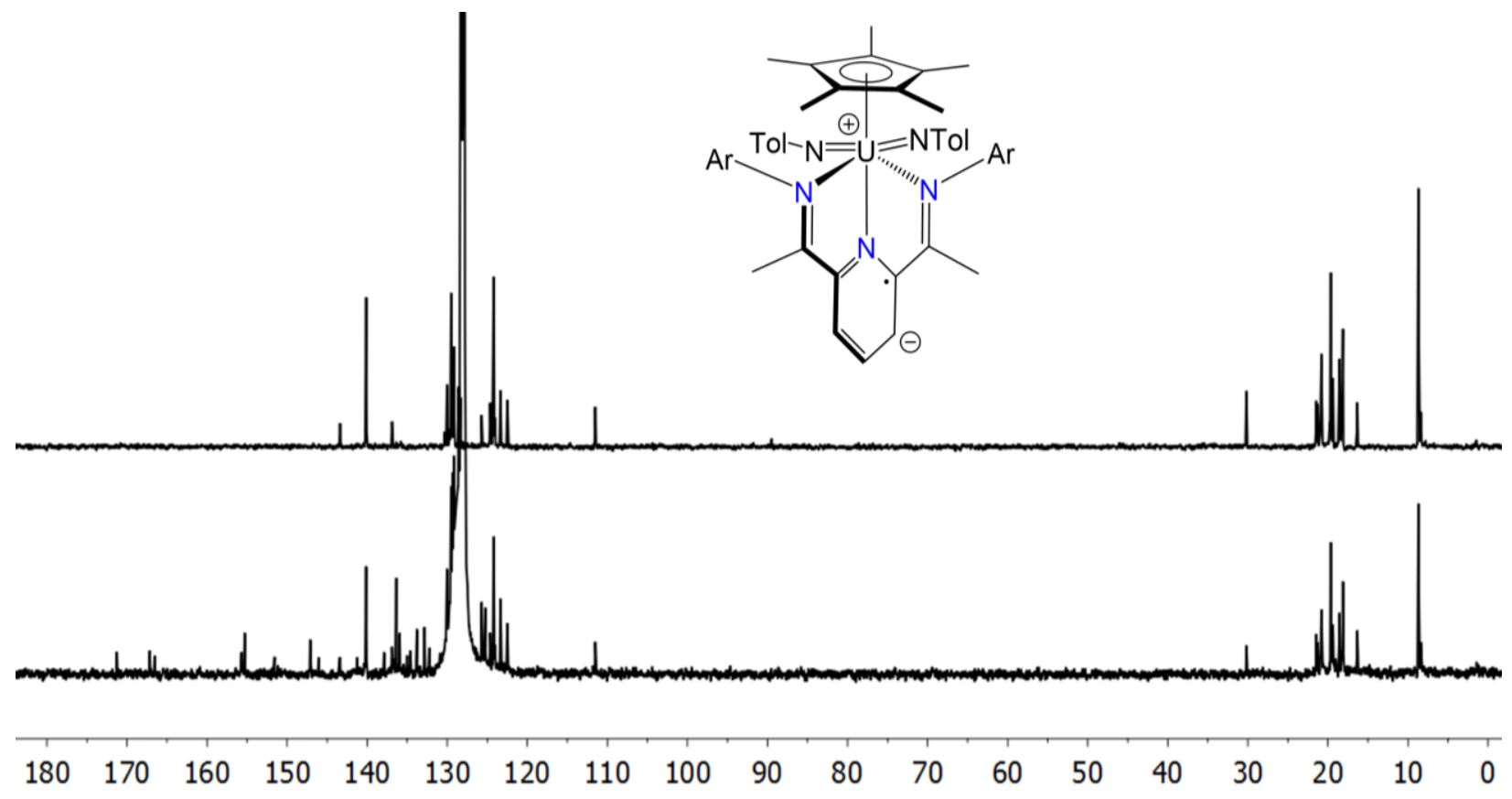

Figure $\mathrm{S} 7{ }^{13} \mathrm{C}$ (bottom) and DEPT-135 (top) spectra (in ppm) of 3-Cp* recorded in $\mathrm{C}_{6} \mathrm{D}_{6}$ at ambient temperature

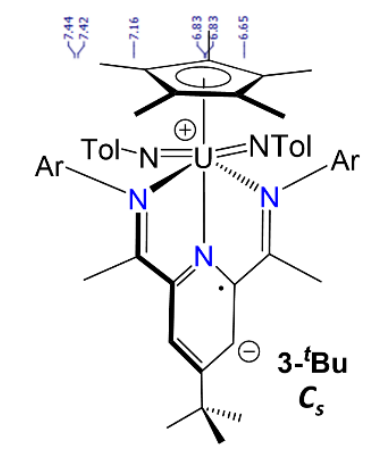

\section{䯘}

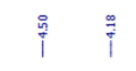

$\stackrel{\sim}{i}$
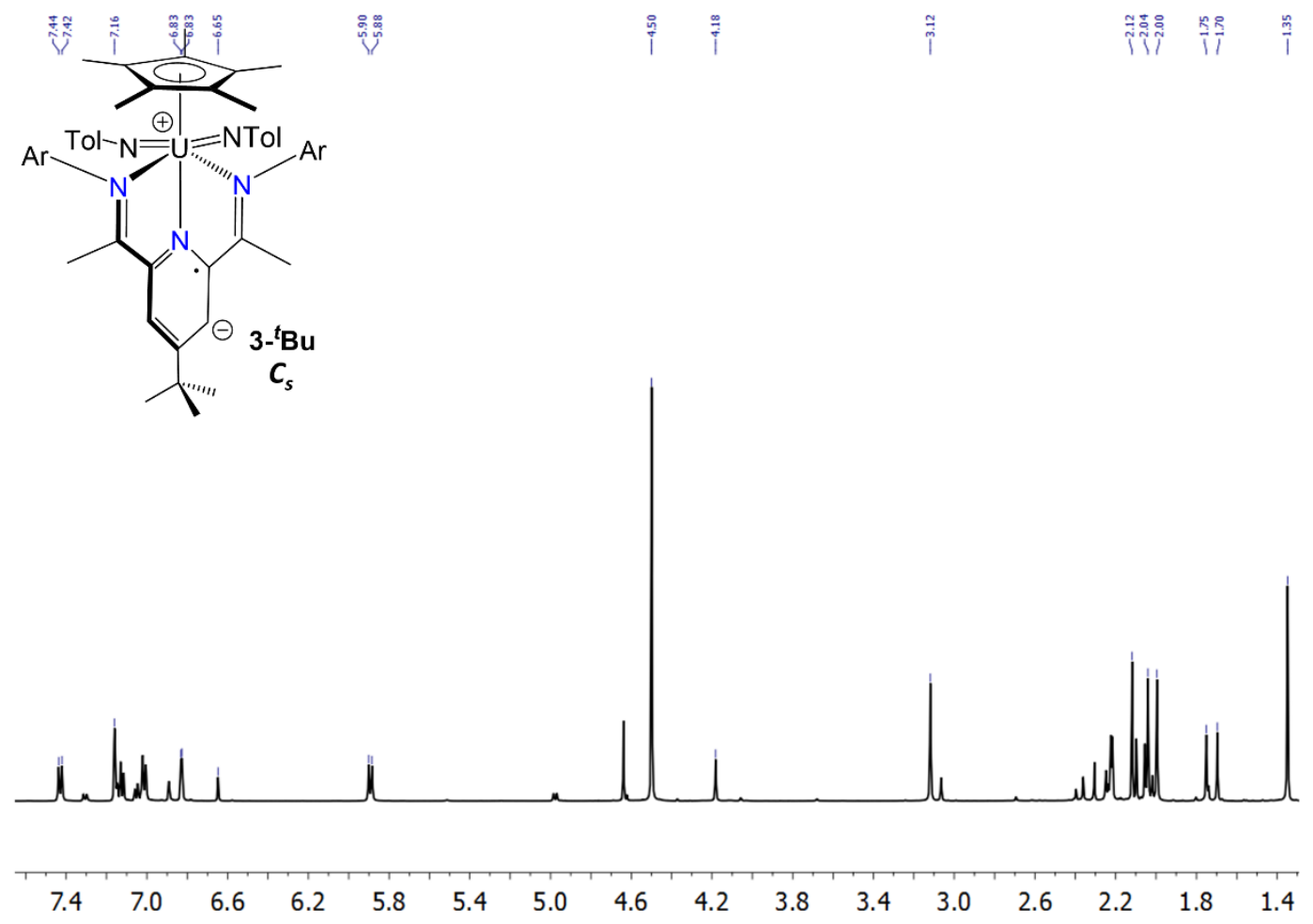

Figure S8 ${ }^{1} \mathrm{H}$ NMR spectrum $\left(\mathrm{C}_{6} \mathrm{D}_{6}, 500 \mathrm{MHz}, 25^{\circ} \mathrm{C}\right)$ of $\mathbf{3}^{-}{ }^{-} \mathbf{B u}$ 


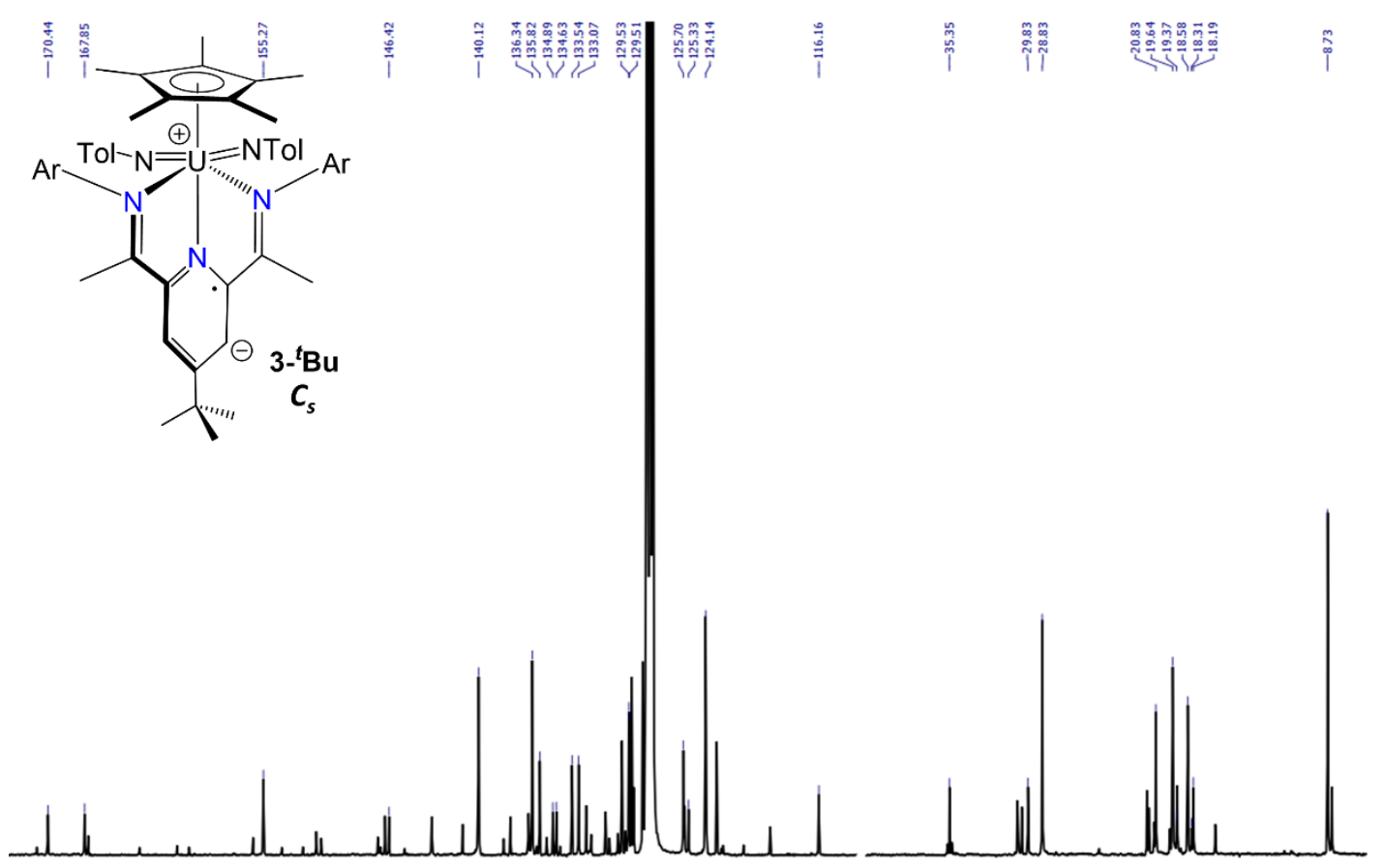

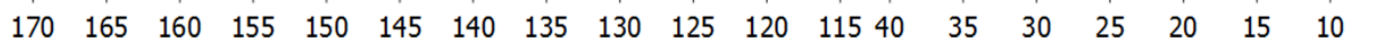

Figure $S 9{ }^{13} \mathrm{C}$ NMR spectrum $\left(\mathrm{C}_{6} \mathrm{D}_{6}, 126 \mathrm{MHz}, 25^{\circ} \mathrm{C}\right)$ of $\mathbf{3}^{-{ }^{-}} \mathbf{B u}$

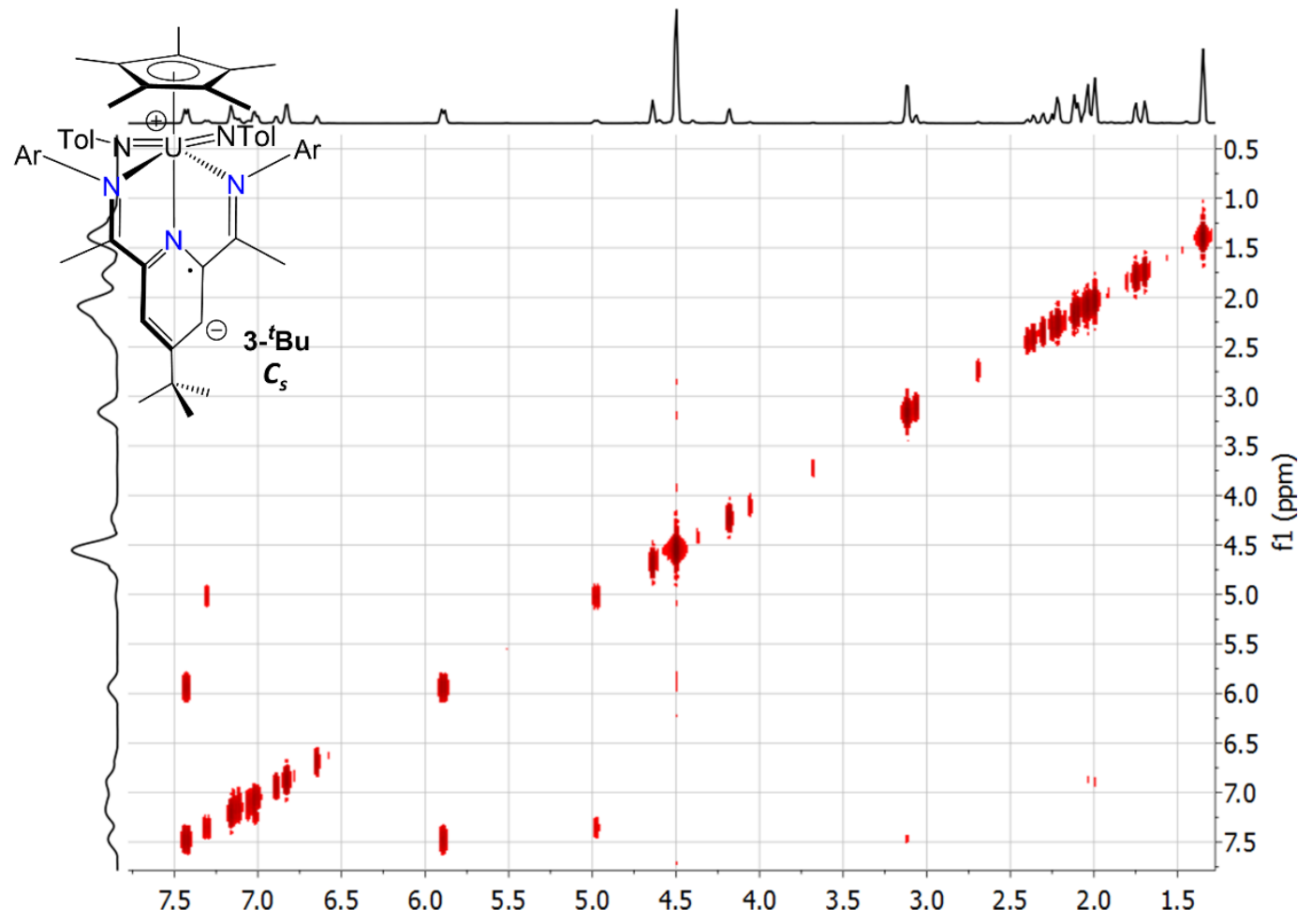

Figure S10 ${ }^{1} \mathrm{H}-{ }^{-1} \mathrm{H}$ COSY spectrum $\left(\mathrm{C}_{6} \mathrm{D}_{6}, 500 \mathrm{MHz}, 25^{\circ} \mathrm{C}\right)$ of $3-{ }^{-t} \mathrm{Bu}$ 


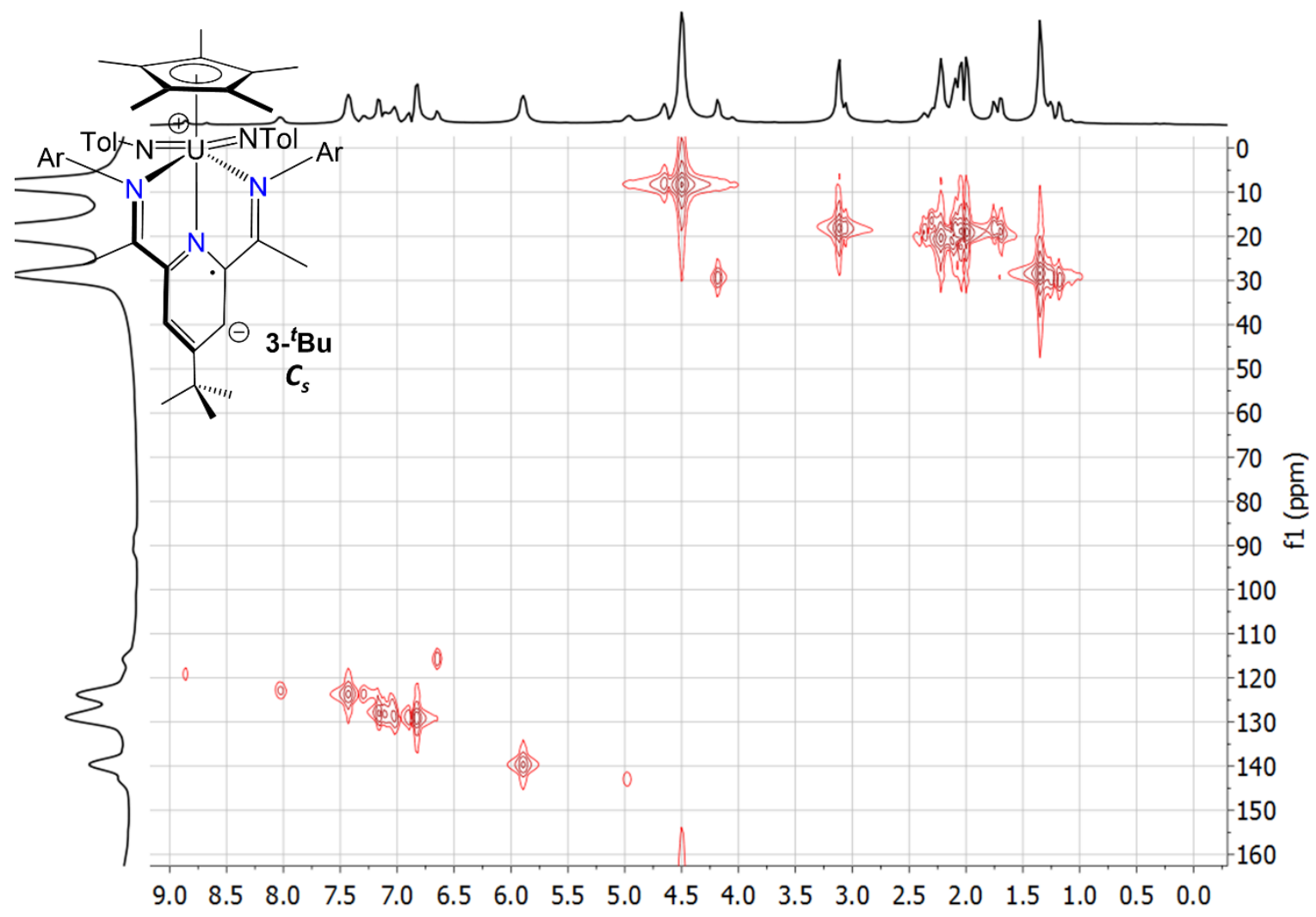

Figure S11 HMQC spectrum $\left(\mathrm{C}_{6} \mathrm{D}_{6}, 126 \& 500 \mathrm{MHz}, 25^{\circ} \mathrm{C}\right)$ of $\mathbf{3 -}{ }^{-} \mathbf{B u}$
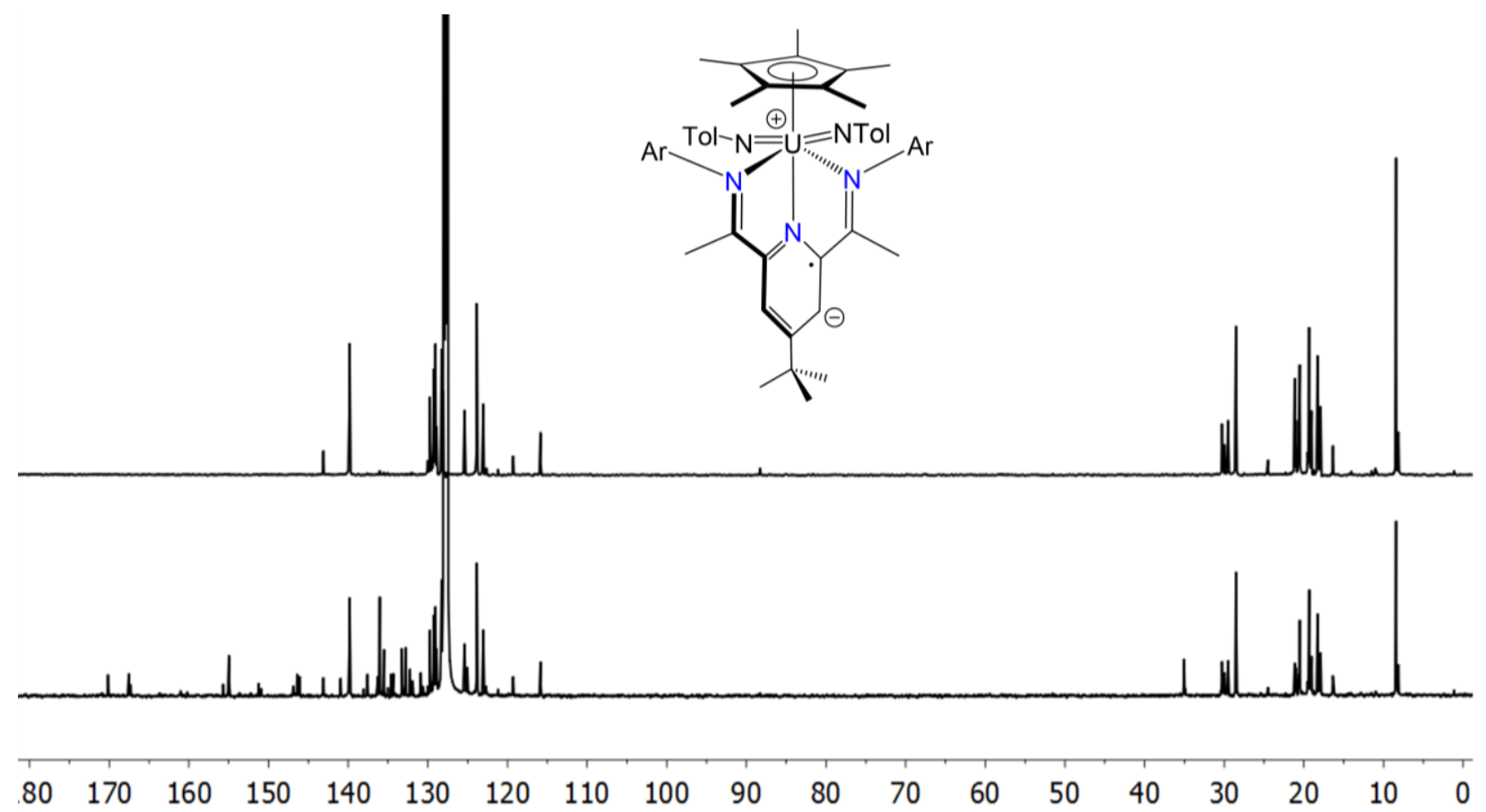

Figure $\mathrm{S} 12{ }^{13} \mathrm{C}$ (bottom) and DEPT-135 (top) spectra (in ppm) of $3-{ }^{-} \mathrm{Bu}$ recorded in $\mathrm{C}_{6} \mathrm{D}_{6}$ at ambient temperature 


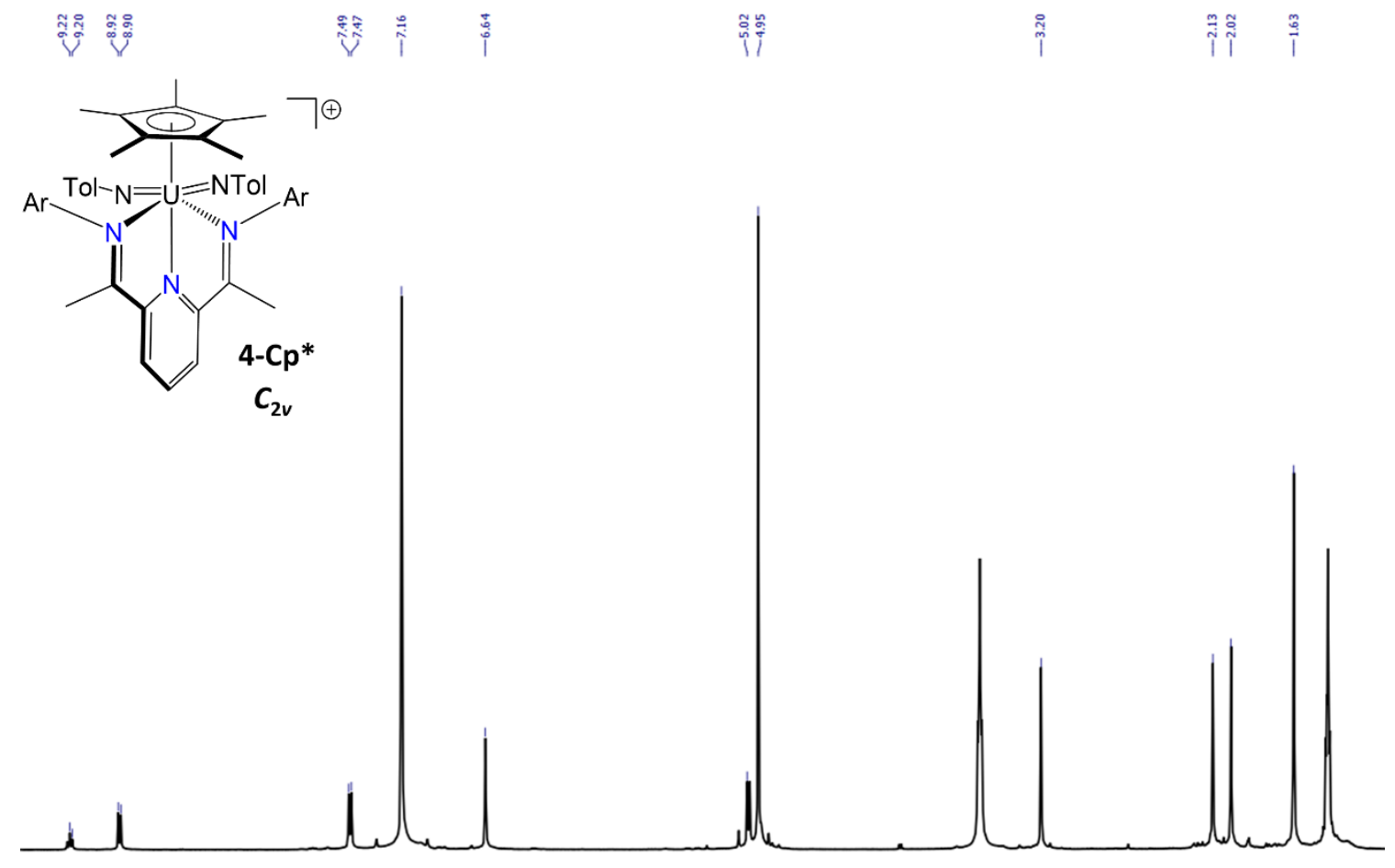

$\begin{array}{lllllllllllllllll}.5 & 9.0 & 8.5 & 8.0 & 7.5 & 7.0 & 6.5 & 6.0 & 5.5 & 5.0 & 4.5 & 4.0 & 3.5 & 3.0 & 2.5 & 2.0 & 1.5\end{array}$

Figure $\mathrm{S} 13{ }^{1} \mathrm{H}$ NMR spectrum $\left(\mathrm{C}_{6} \mathrm{D}_{6}, 500 \mathrm{MHz}, 25{ }^{\circ} \mathrm{C}\right)$ of 4-Cp*

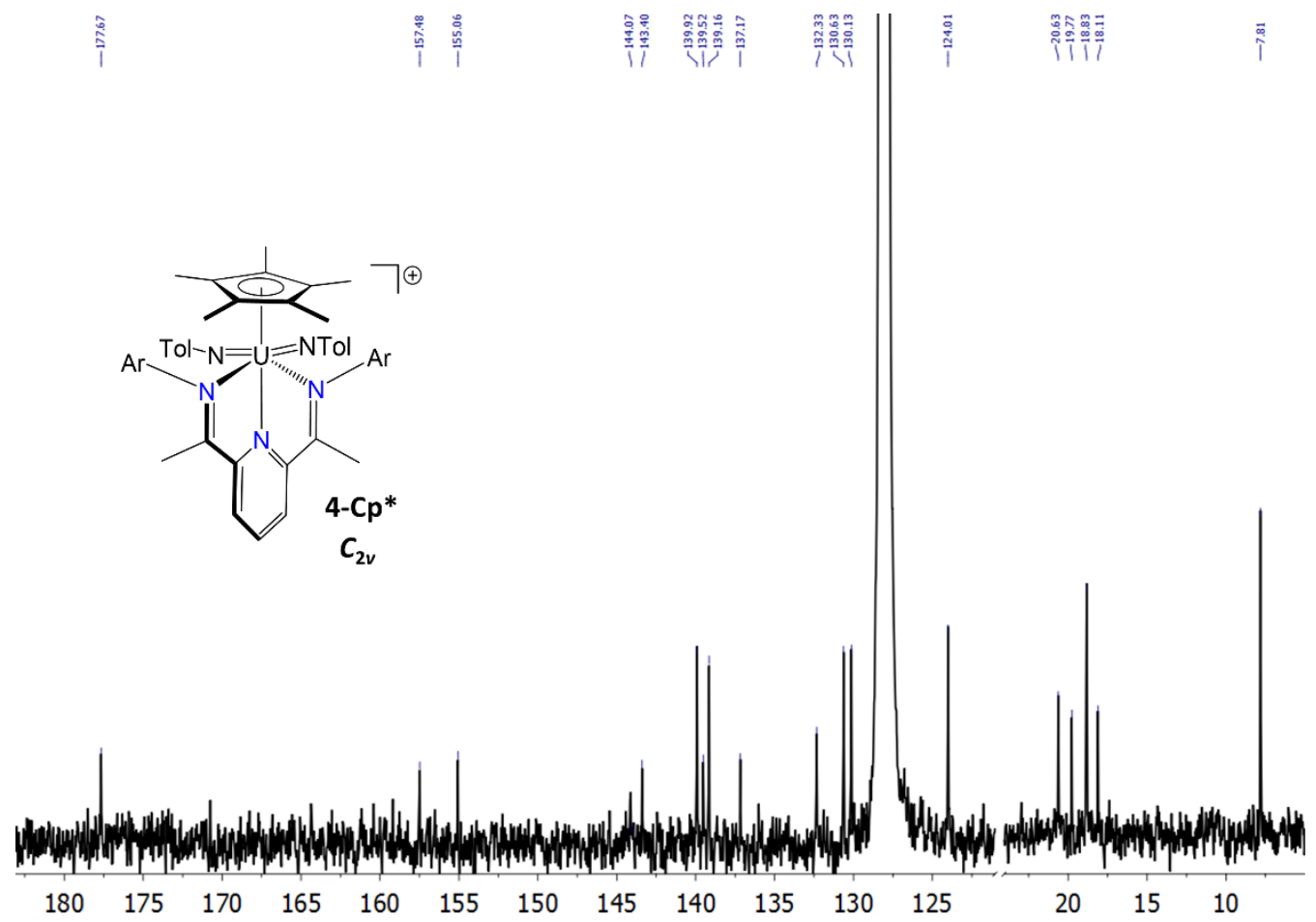

Figure $\mathrm{S} 14{ }^{13} \mathrm{C}$ NMR spectrum $\left(\mathrm{C}_{6} \mathrm{D}_{6}, 126 \mathrm{MHz}, 25^{\circ} \mathrm{C}\right)$ of 4-Cp* 


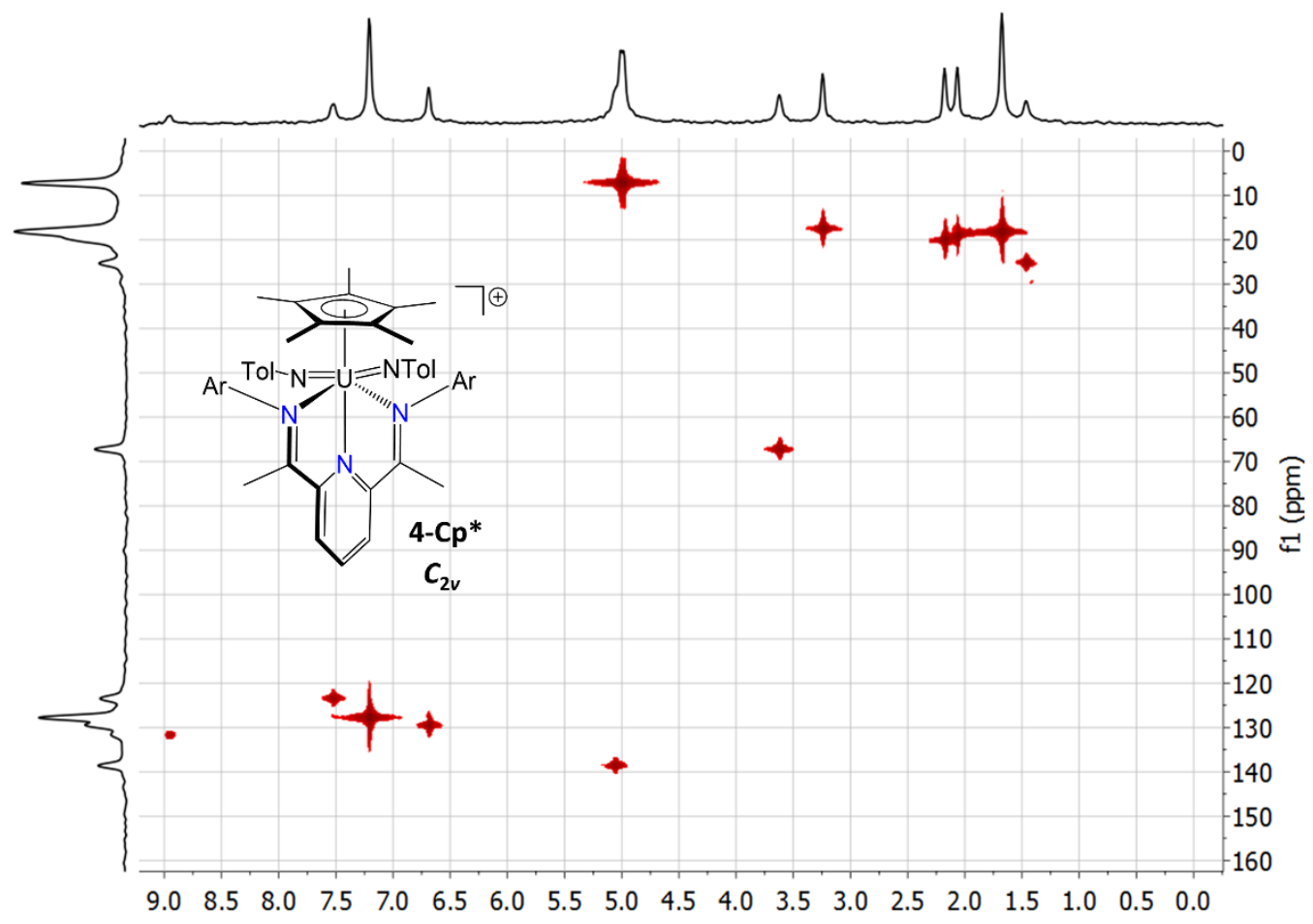

Figure S14 HMQC spectrum $\left(\mathrm{C}_{6} \mathrm{D}_{6}, 126 \& 500 \mathrm{MHz}, 25{ }^{\circ} \mathrm{C}\right)$ of 4-Cp*

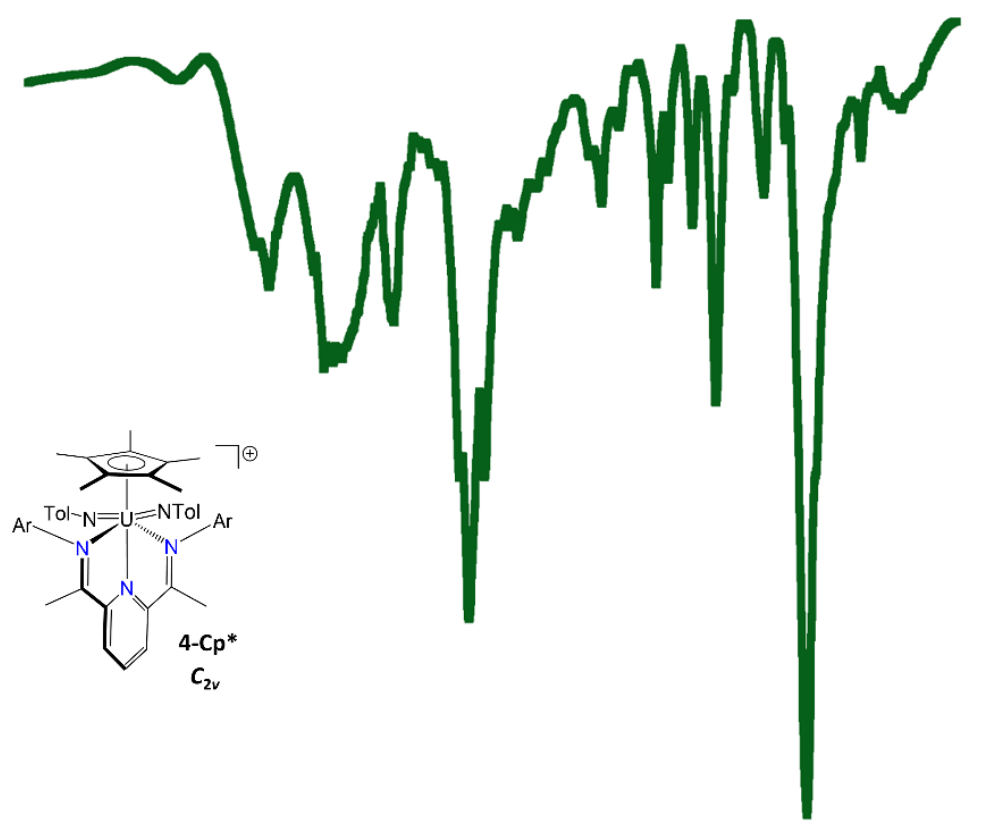

\section{0 Wavenumber, $\mathrm{cm}^{-1}$}

Figure S16 Infrared spectrum (Salt Plate, KBr) of 4-Cp* 


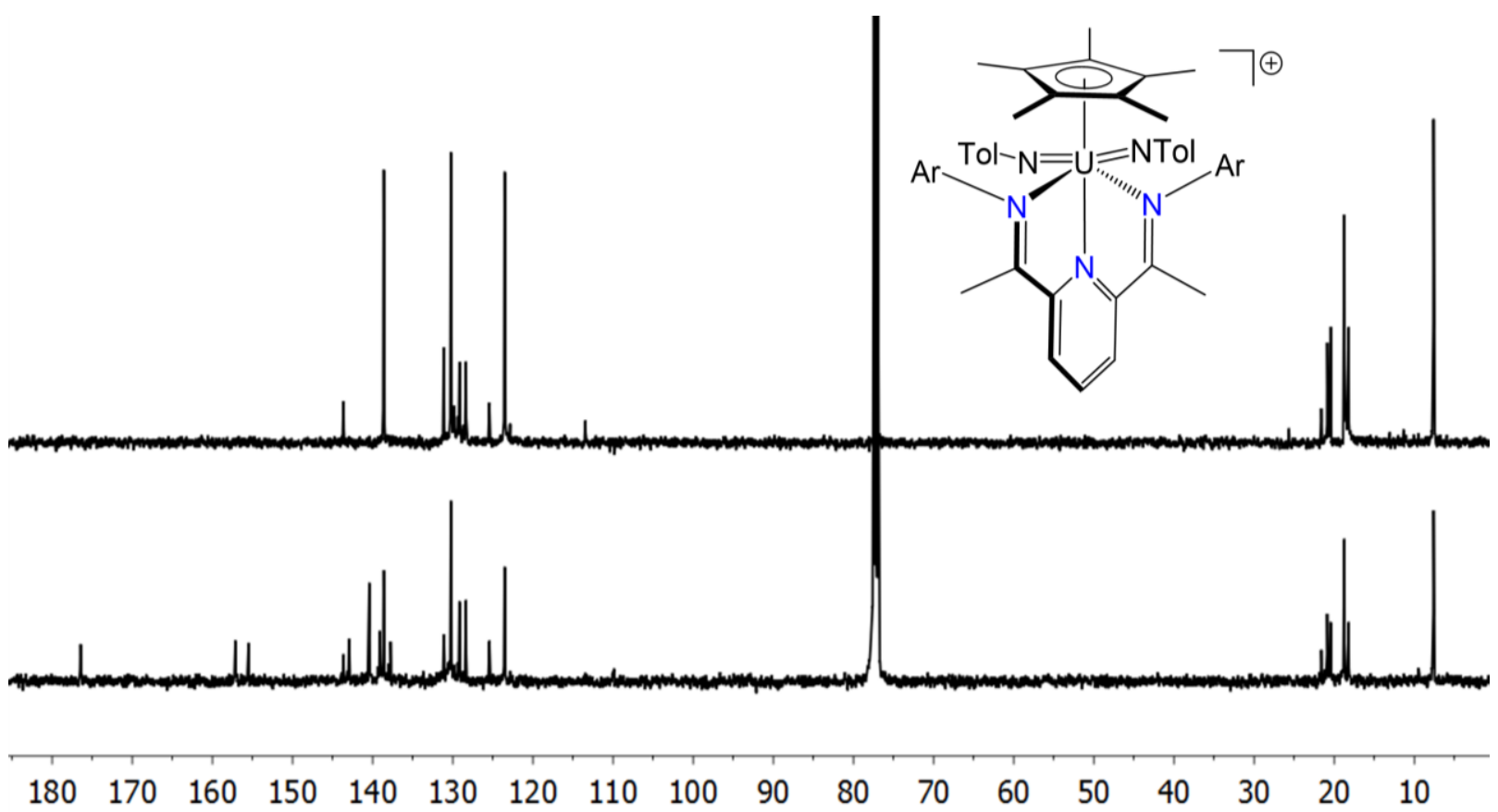

Figure $\mathrm{S} 17{ }^{13} \mathrm{C}$ (bottom) and DEPT-135 (top) spectra (in ppm) of 4-Cp* recorded in $\mathrm{CDCl}_{3}$ at ambient temperature.

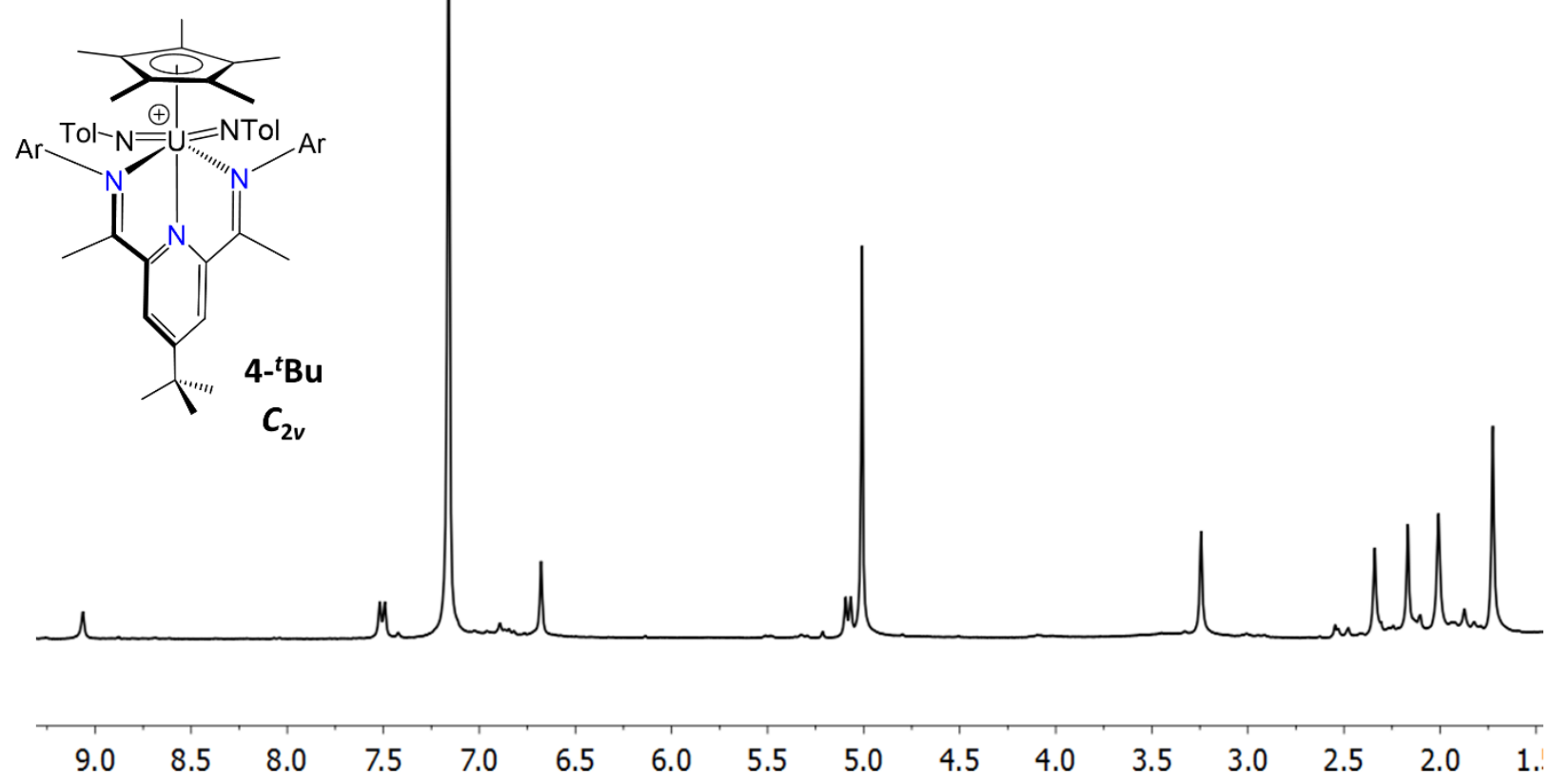

Figure S18 ${ }^{1} \mathrm{H}$ NMR spectrum $\left(\mathrm{C}_{6} \mathrm{D}_{6}, 300 \mathrm{MHz}, 25^{\circ} \mathrm{C}\right)$ of $\mathbf{4}^{-{ }^{-}} \mathbf{B u}$ 


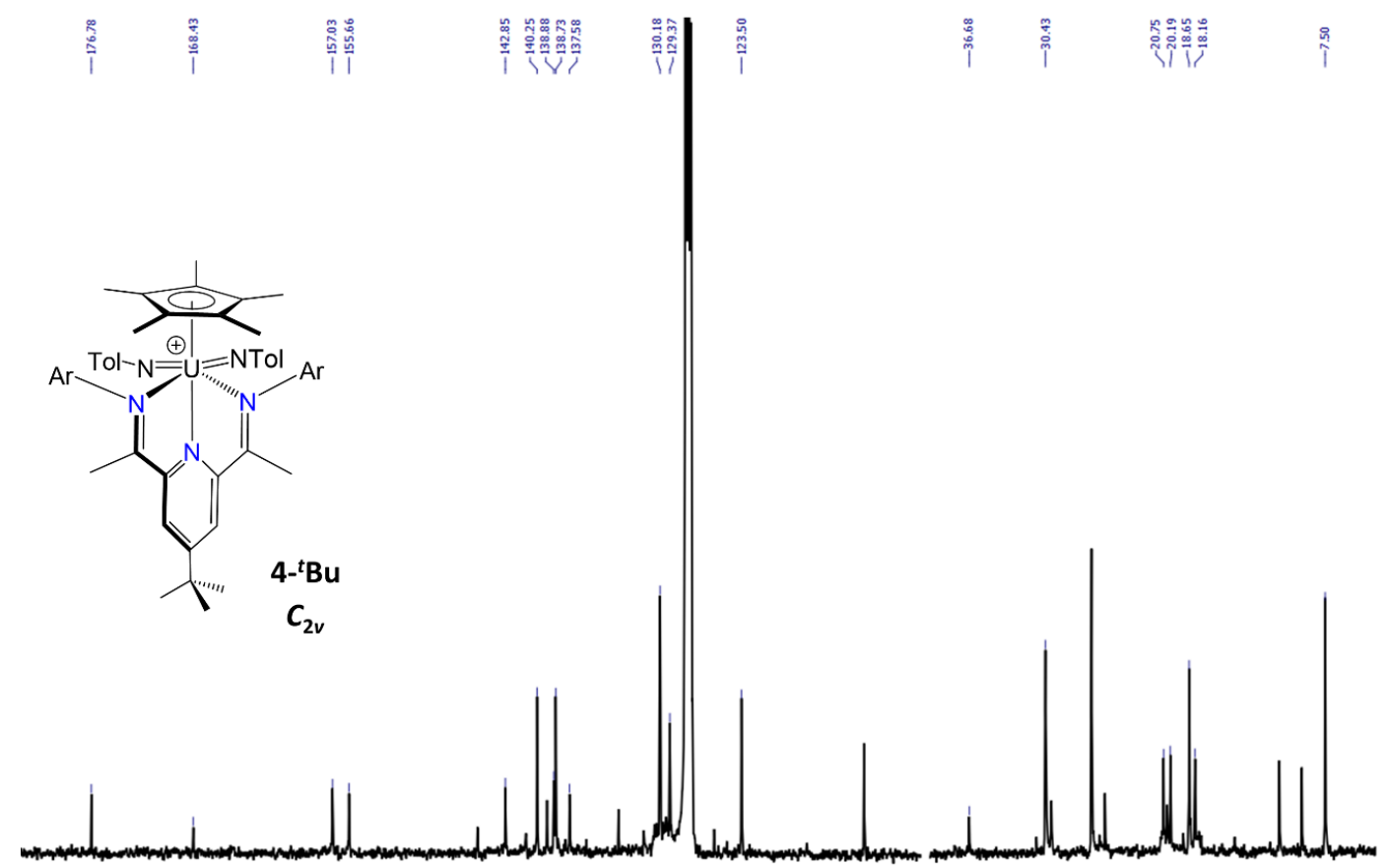

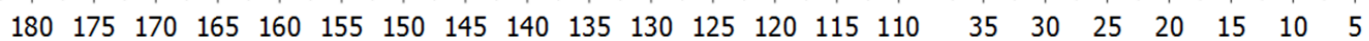

Figure $\mathrm{S} 19{ }^{13} \mathrm{C}$ NMR spectrum $\left(\mathrm{CDCl}_{3}, 126 \mathrm{MHz}, 25^{\circ} \mathrm{C}\right)$ of $\mathbf{4}^{-t} \mathbf{B u}$

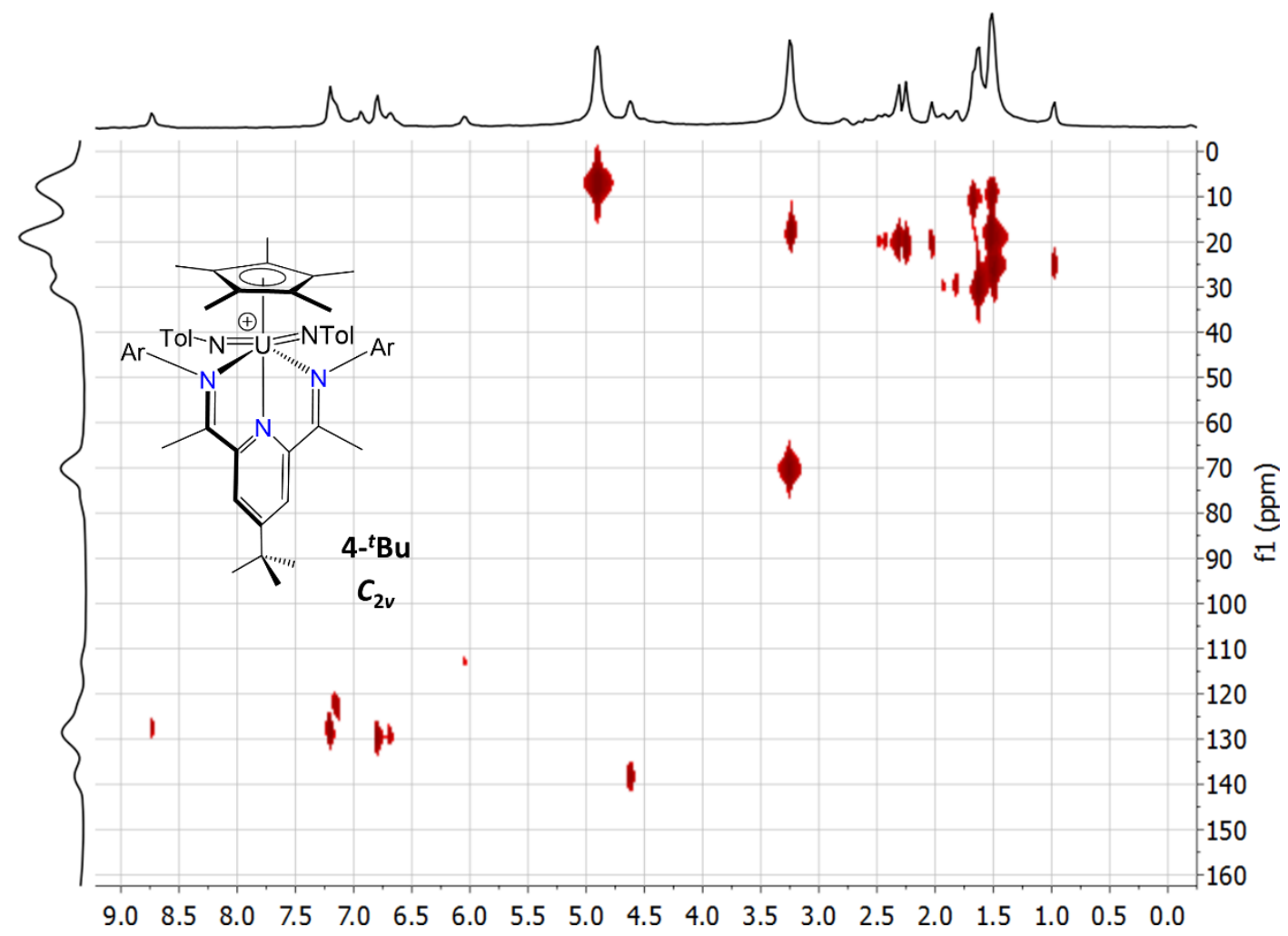

Figure S20 HMQC spectrum $\left(\mathrm{CDCl}_{3}, 126 \& 500 \mathrm{MHz}, 25^{\circ} \mathrm{C}\right)$ of 4-t $\mathbf{B u}$ 


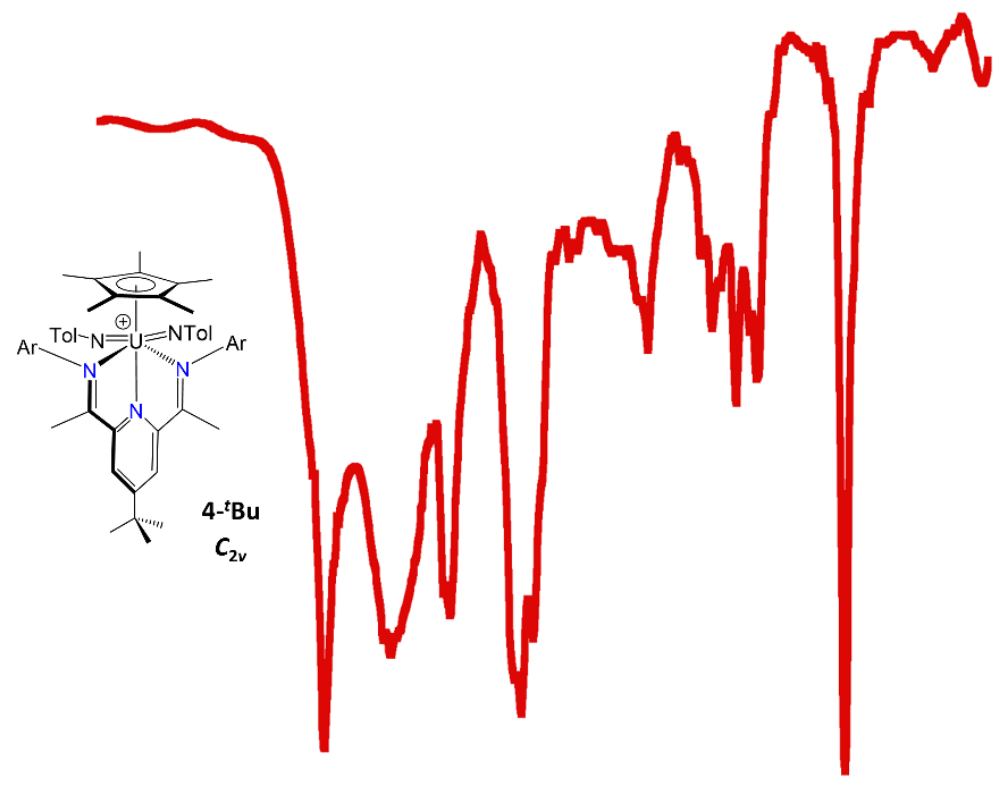

\section{0 \\ Wavenumber, $\mathrm{cm}^{-1}$}

Figure S21 Infrared spectrum (Salt Plate, KBr) of 4-tBu

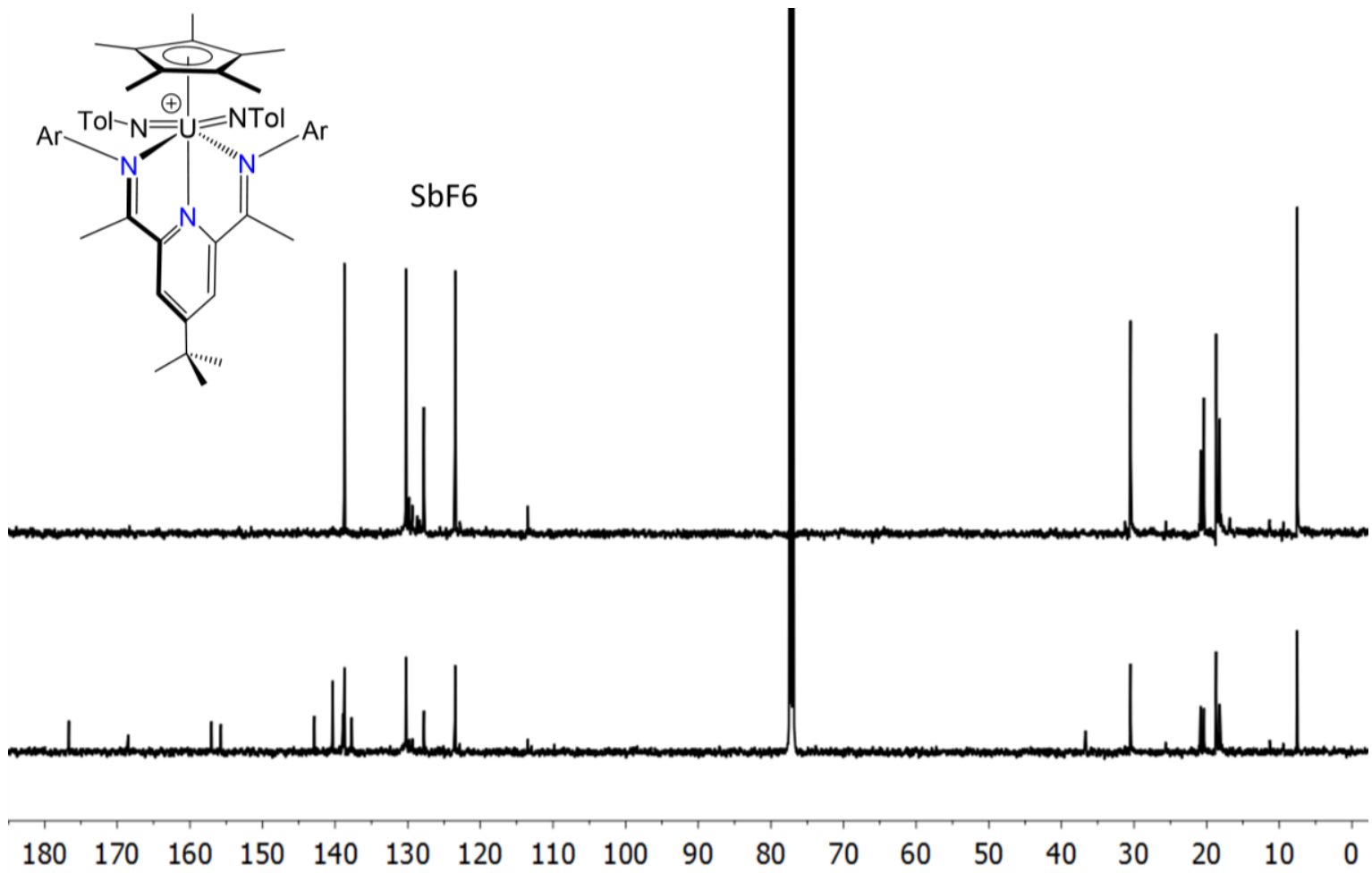

Figure S22 ${ }^{13} \mathrm{C}$ (bottom) and DEPT-135 (top) spectra (in ppm) of 4- ${ }^{-} \mathbf{B u}$ recorded in $\mathrm{CDCl}_{3}$ at ambient temperature. 

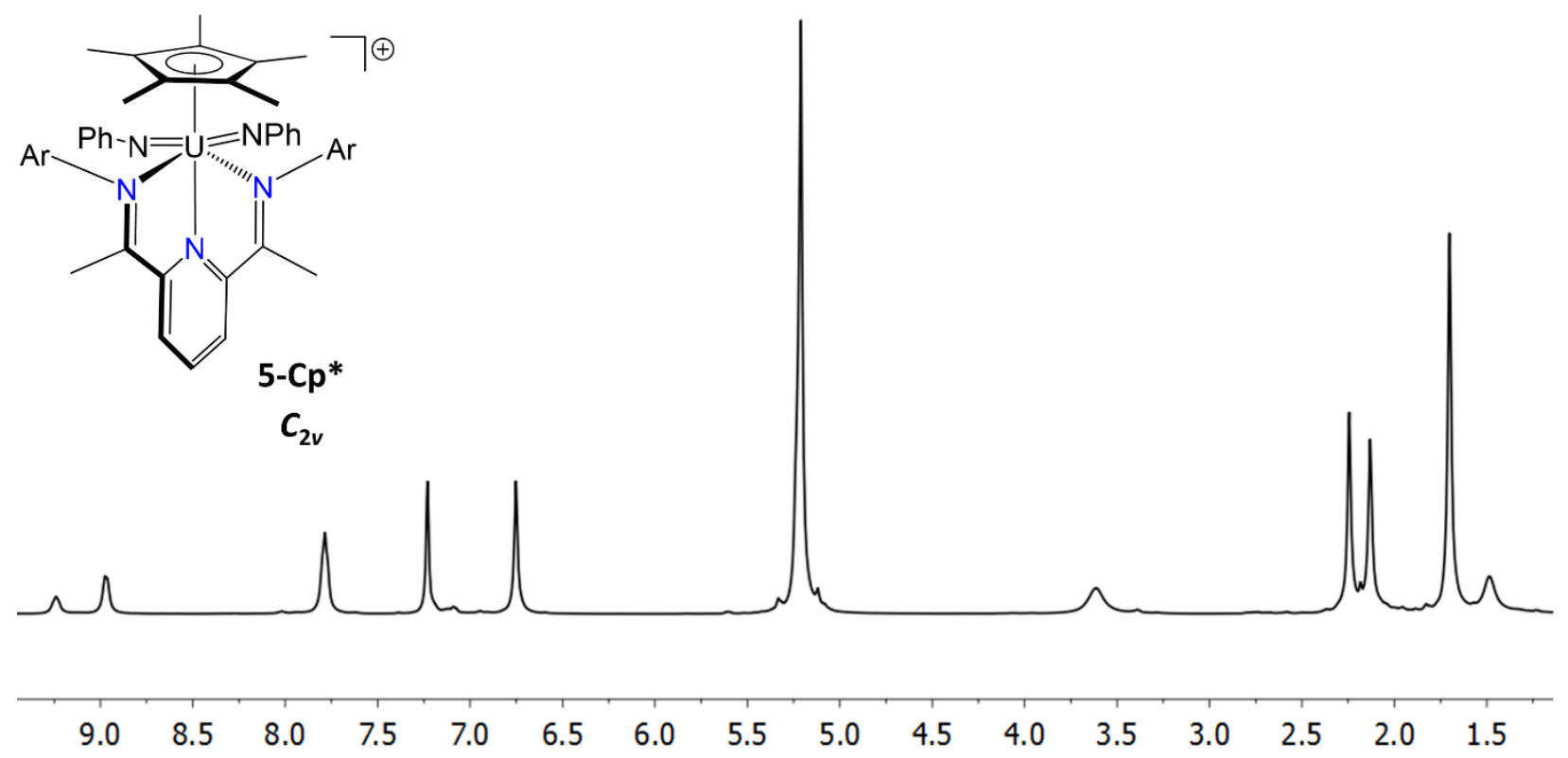

Figure $\mathrm{S} 23{ }^{1} \mathrm{H}$ NMR spectrum $\left(\mathrm{C}_{6} \mathrm{D}_{6}, 500 \mathrm{MHz}, 25^{\circ} \mathrm{C}\right)$ of $\mathbf{5}-\mathbf{C p}^{*}$

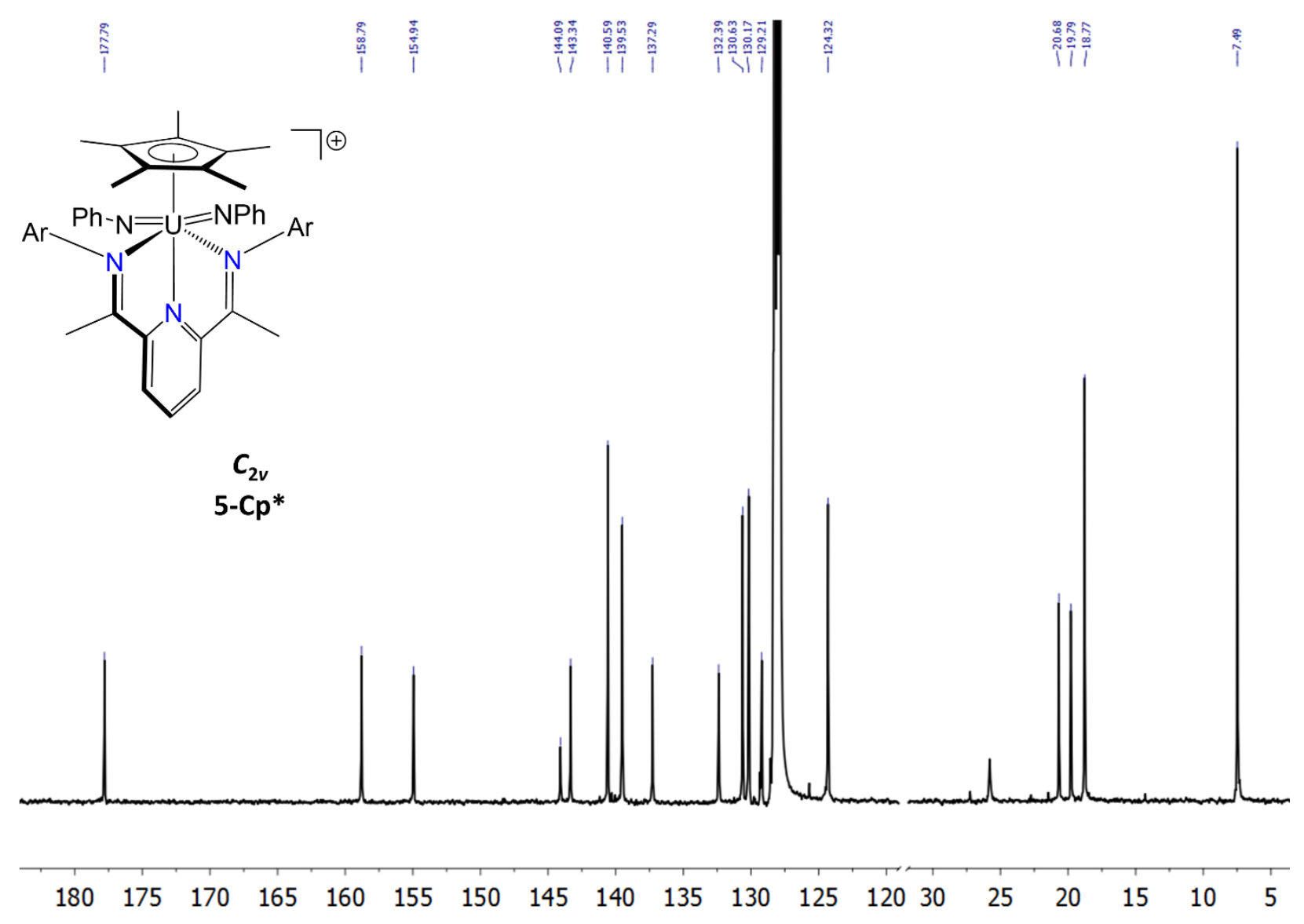

Figure $\mathrm{S} 24{ }^{13} \mathrm{C}$ NMR spectrum $\left(\mathrm{C}_{6} \mathrm{D}_{6}, 126 \mathrm{MHz}, 25^{\circ} \mathrm{C}\right)$ of $\mathbf{5}-\mathbf{C p} *$ 


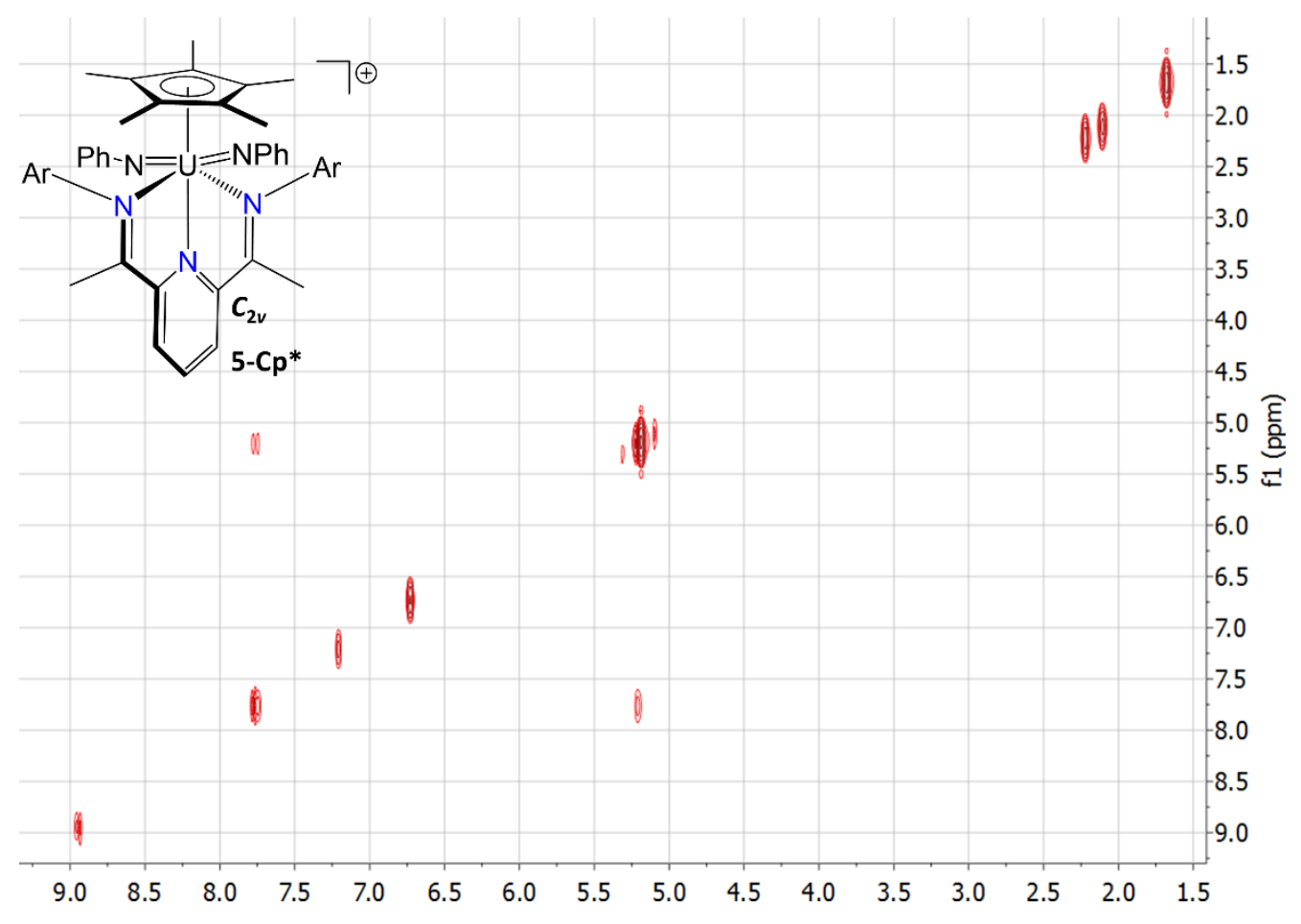

Figure $\mathrm{S} 25{ }^{1} \mathrm{H}-{ }^{1} \mathrm{H}$ COSY spectrum $\left(\mathrm{C}_{6} \mathrm{D}_{6}, 500 \mathrm{MHz}, 25^{\circ} \mathrm{C}\right)$ of $\mathbf{5}-\mathbf{C p} *$

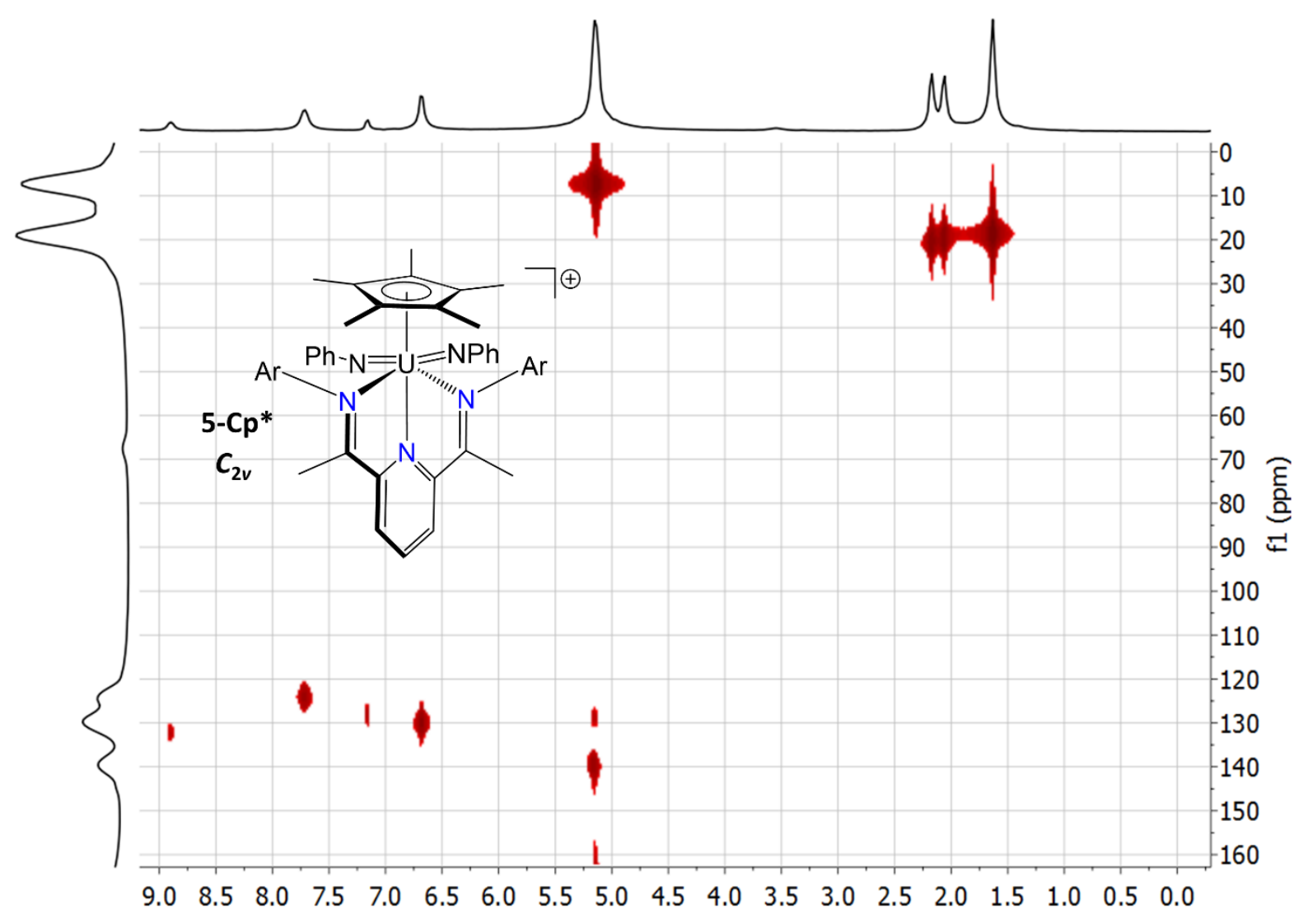

Figure S26 HMQC spectrum $\left(\mathrm{C}_{6} \mathrm{D}_{6}, 126 \& 500 \mathrm{MHz}, 25^{\circ} \mathrm{C}\right)$ of $\mathbf{5}-\mathbf{C p}$ * 


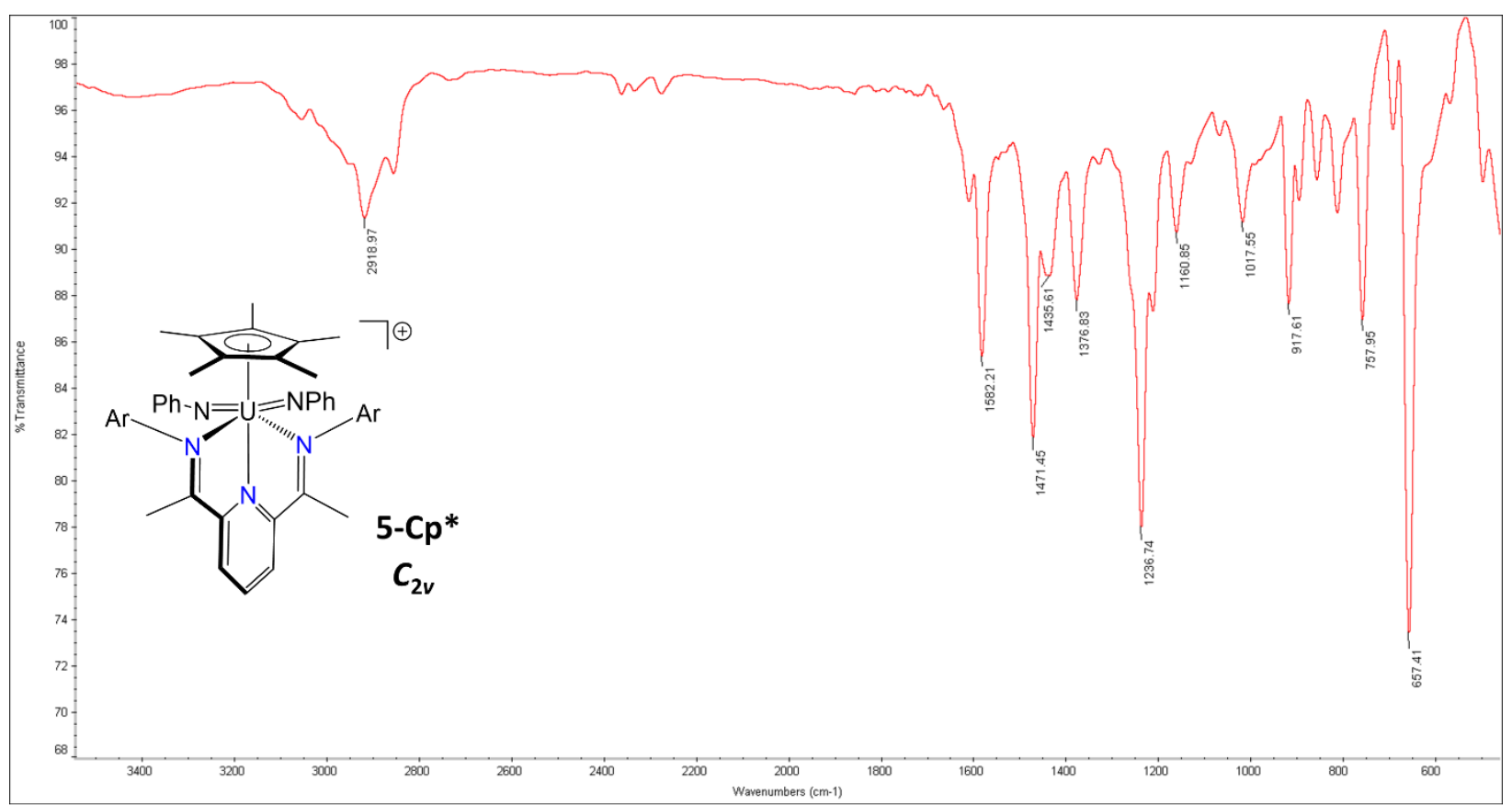

Figure S27 Infrared spectrum (Salt Plate, KBr) of 5-Cp*

$*$ = residual tetrahydrofuran

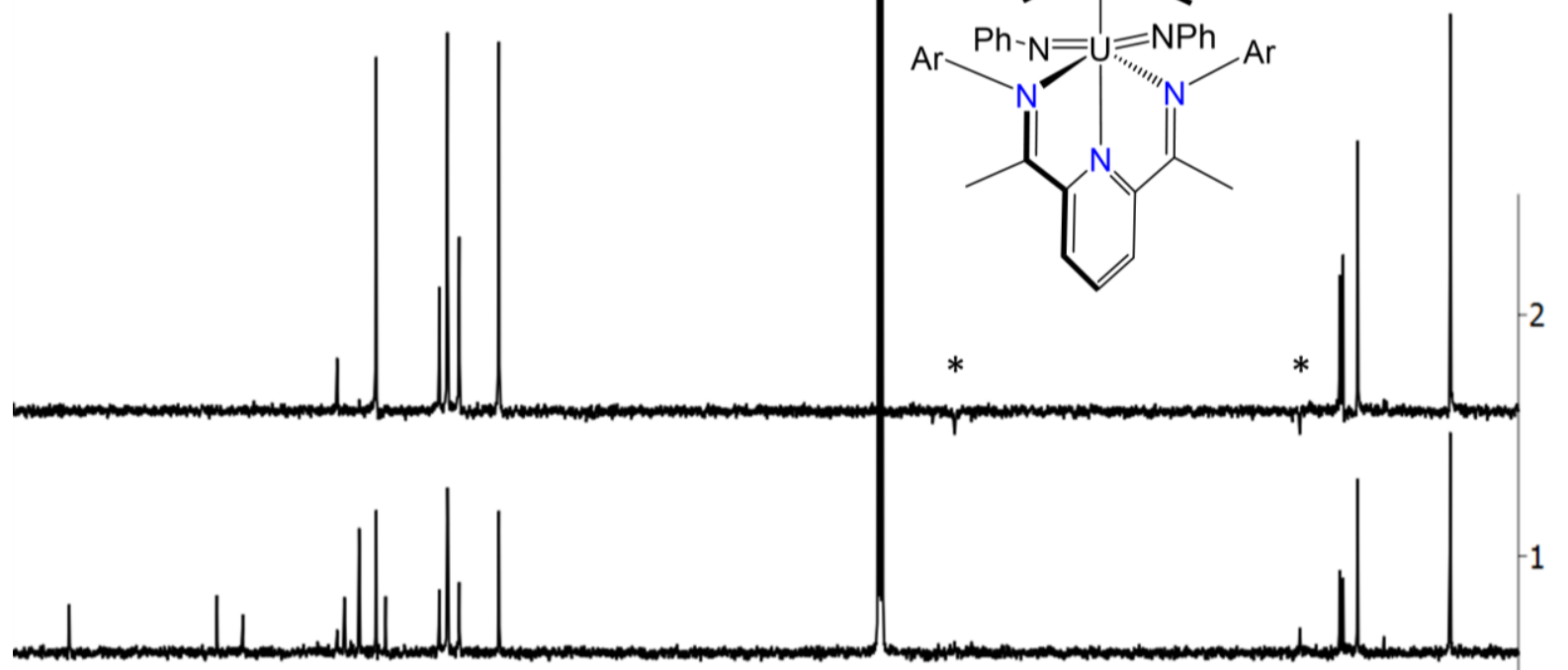

$\begin{array}{lllllllllllllllllll}180 & 170 & 160 & 150 & 140 & 130 & 120 & 110 & 100 & 90 & 80 & 70 & 60 & 50 & 40 & 30 & 20 & 10 & 0\end{array}$

Figure S28 ${ }^{13} \mathrm{C}$ (bottom) and DEPT-135 (top) spectra (in ppm) of 5-Cp* recorded in $\mathrm{CDCl}_{3}$ at ambient temperature. Asterisk (*) denotes residual tetrahydrofuran. 


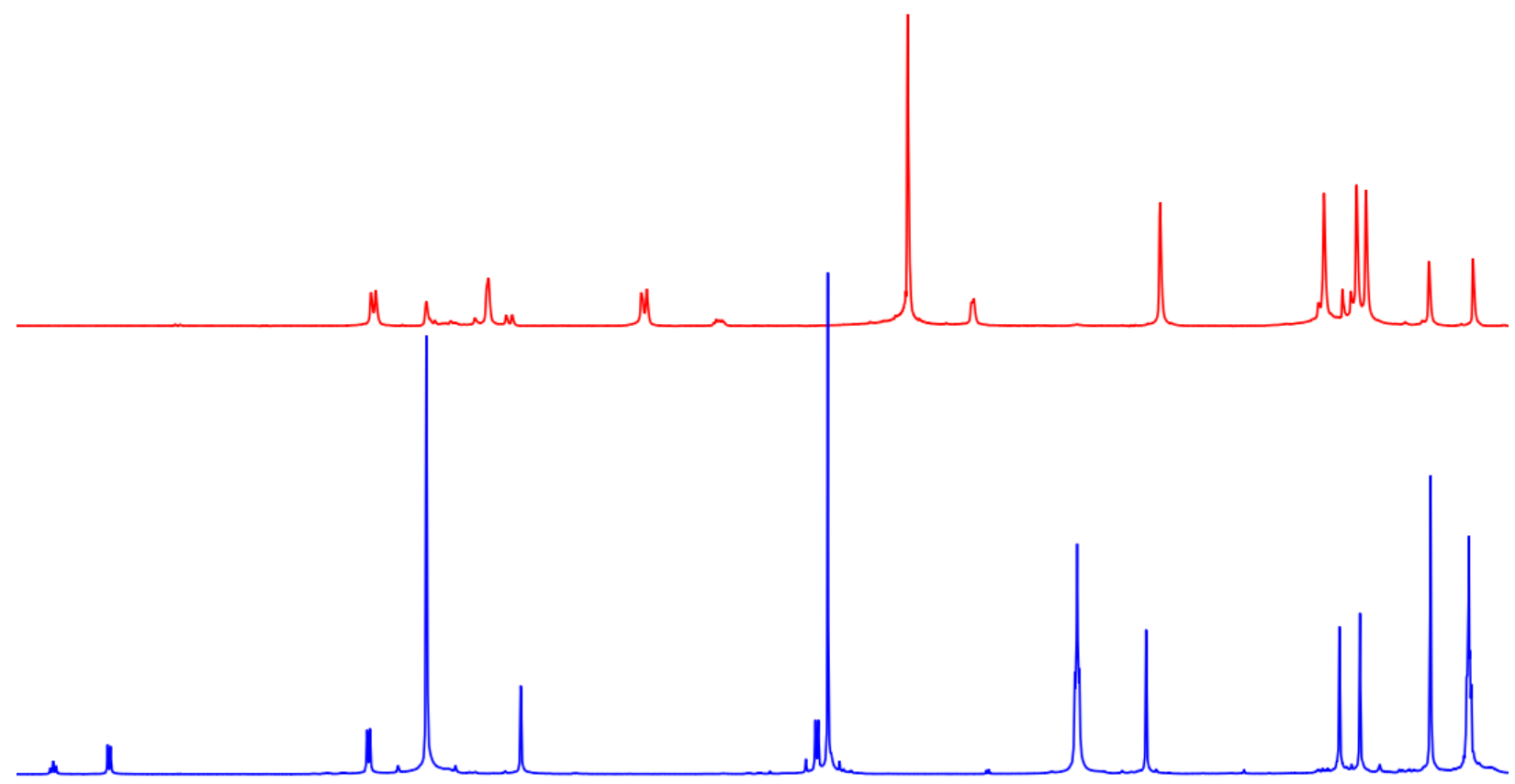

$\begin{array}{llllllllllllllll}9.0 & 8.5 & 8.0 & 7.5 & 7.0 & 6.5 & 6.0 & 5.5 & 5.0 & 4.5 & 4.0 & 3.5 & 3.0 & 2.5 & 2.0 & 1.5\end{array}$

Figure S29 ${ }^{1}$ H NMR spectra of 3-Cp* (top) and 4-Cp* (bottom) with residual solvent.
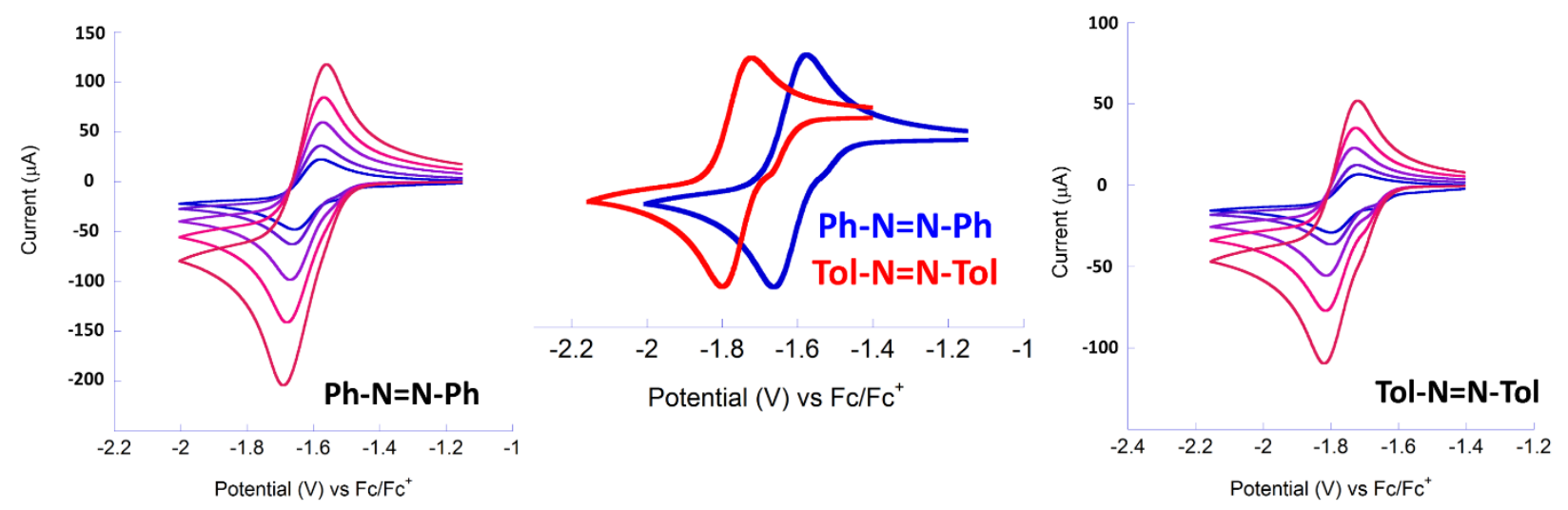

Figure S30 Left: First reduction of azobenzene. Sweeps obtained at 50, 100, 250, 500, and $1000 \mathrm{mv} / \mathrm{s}$ (blue to red, respectively) (Conditions: ambient temperature, $5.49 \times 10^{-3} \mathrm{M}$ in

$\mathrm{MeCN}, 0.1 \mathrm{M} \mathrm{Bu}_{4} \mathrm{NPF}_{6}$ ). Middle: Comparison of first reduction potential $(100 \mathrm{mv} / \mathrm{s})$ of azobenzene (blue) and Tol-N=N-Tol (red). Right: First reduction of Tol-N=N-Tol with same sweeps rates as $\mathrm{Ph}-\mathrm{N}=\mathrm{N}-\mathrm{Ph}$ (Conditions: ambient temperature, $3.17 \times 10^{-3} \mathrm{M}$ in $\mathrm{MeCN}, 0.1$

$\mathrm{M} \mathrm{Bu}_{4} \mathrm{NPF}_{6}$ ). 

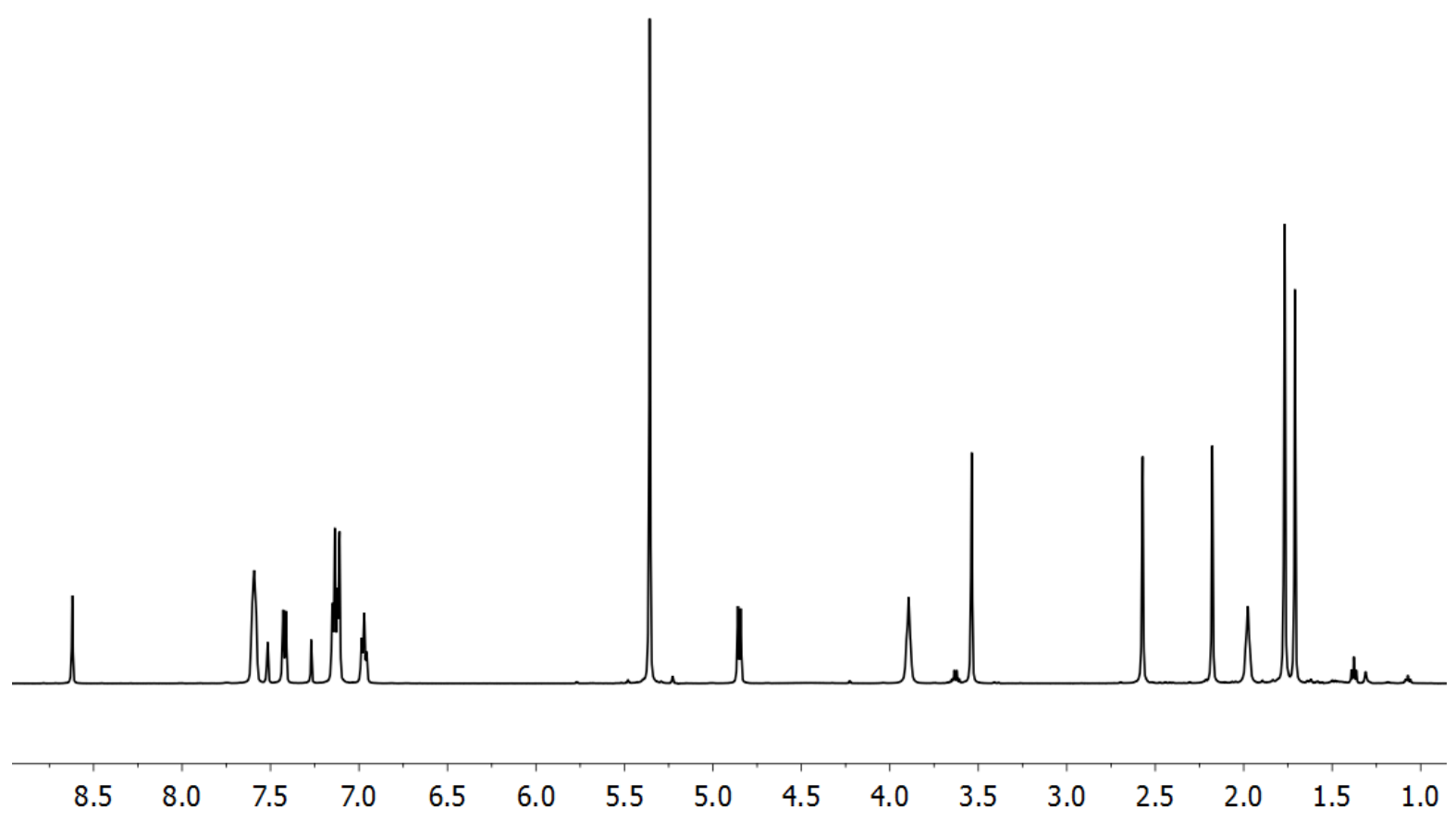

Figure $\mathrm{S} 31{ }^{1} \mathrm{H}$ NMR spectrum $\left(\mathrm{CDCl}_{3}, 500 \mathrm{MHz}, 25^{\circ} \mathrm{C}\right)$ of

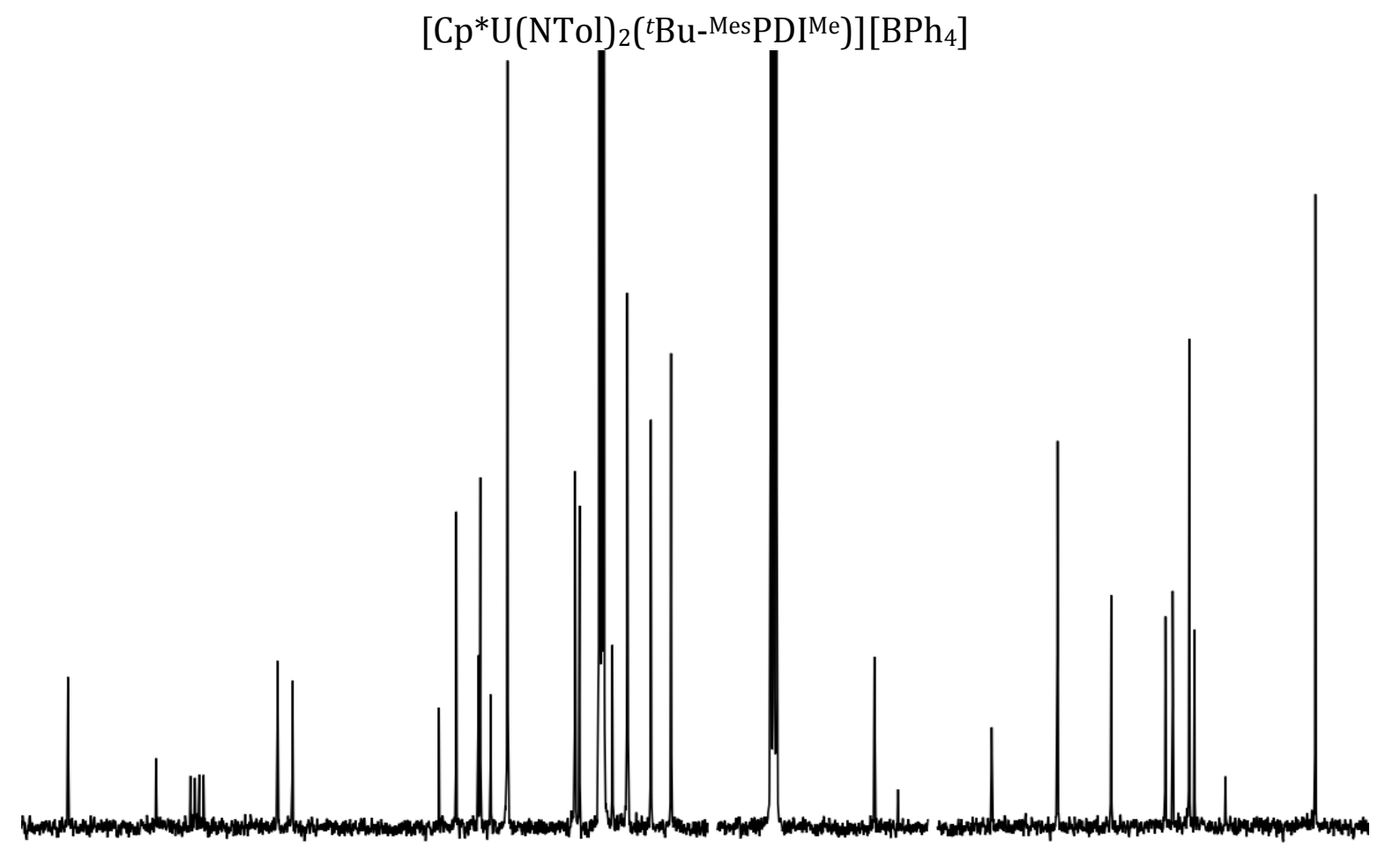

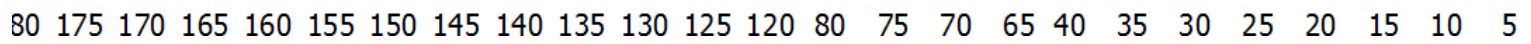

Figure $\mathrm{S} 32{ }^{13} \mathrm{C}$ NMR spectrum $\left(\mathrm{CDCl}_{3}, 126 \mathrm{MHz}, 25{ }^{\circ} \mathrm{C}\right)$ of

$\left[\mathrm{Cp} * \mathrm{U}(\mathrm{NTol})_{2}\left({ }^{\mathrm{t}} \mathrm{Bu}-\mathrm{MesPDI}^{\mathrm{Me}}\right)\right]\left[\mathrm{BPh}_{4}\right]$ 


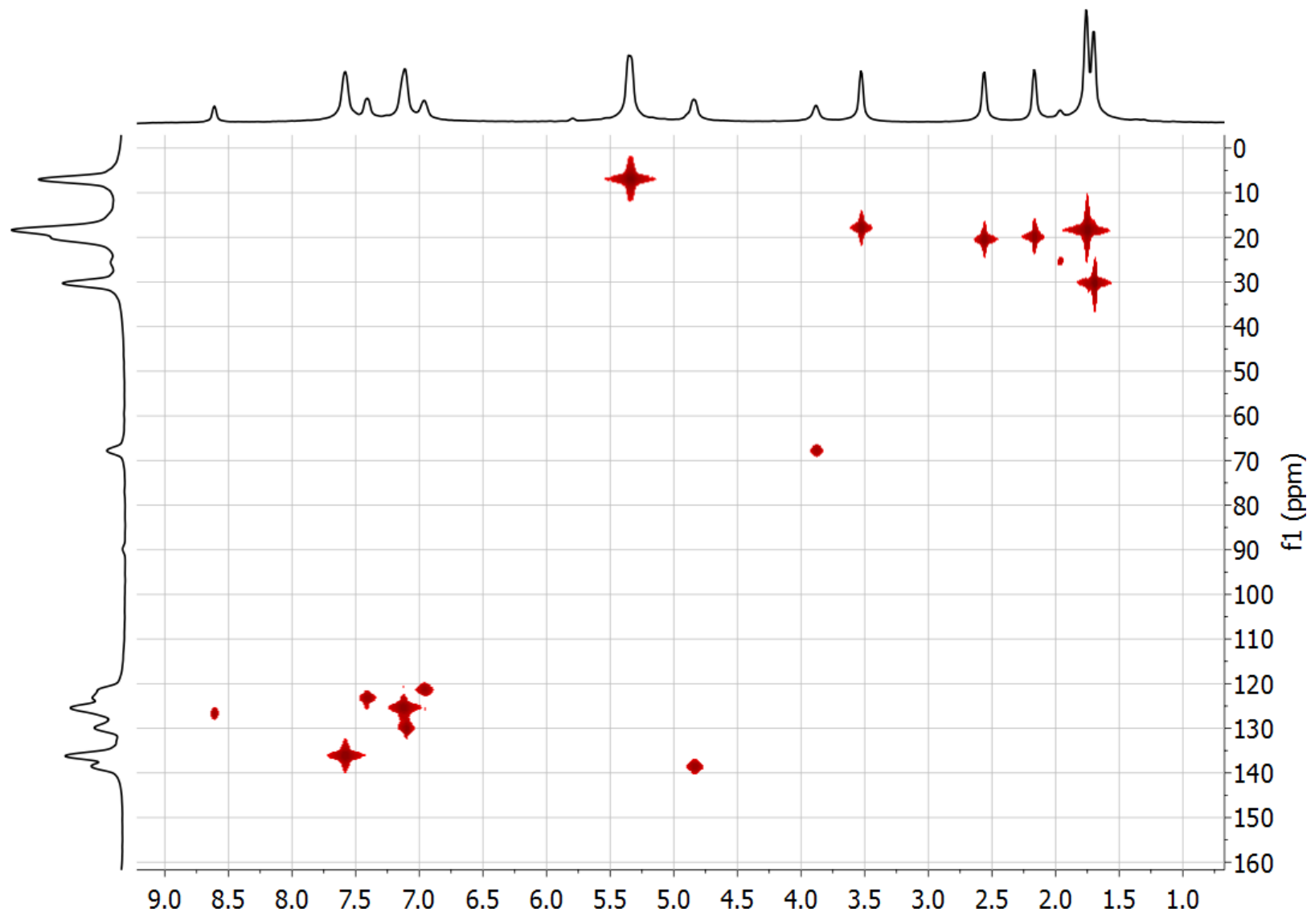

Figure S33 HMQC spectrum $\left(\mathrm{CDCl}_{3}, 126 \& 500 \mathrm{MHz}, 25^{\circ} \mathrm{C}\right)$ of $\left[\mathrm{Cp}^{*} \mathrm{U}(\mathrm{NTol})_{2}\left({ }^{\mathrm{t}} \mathrm{Bu}-\mathrm{MesPDIMe}\right)\right]\left[\mathrm{BPh}_{4}\right]$

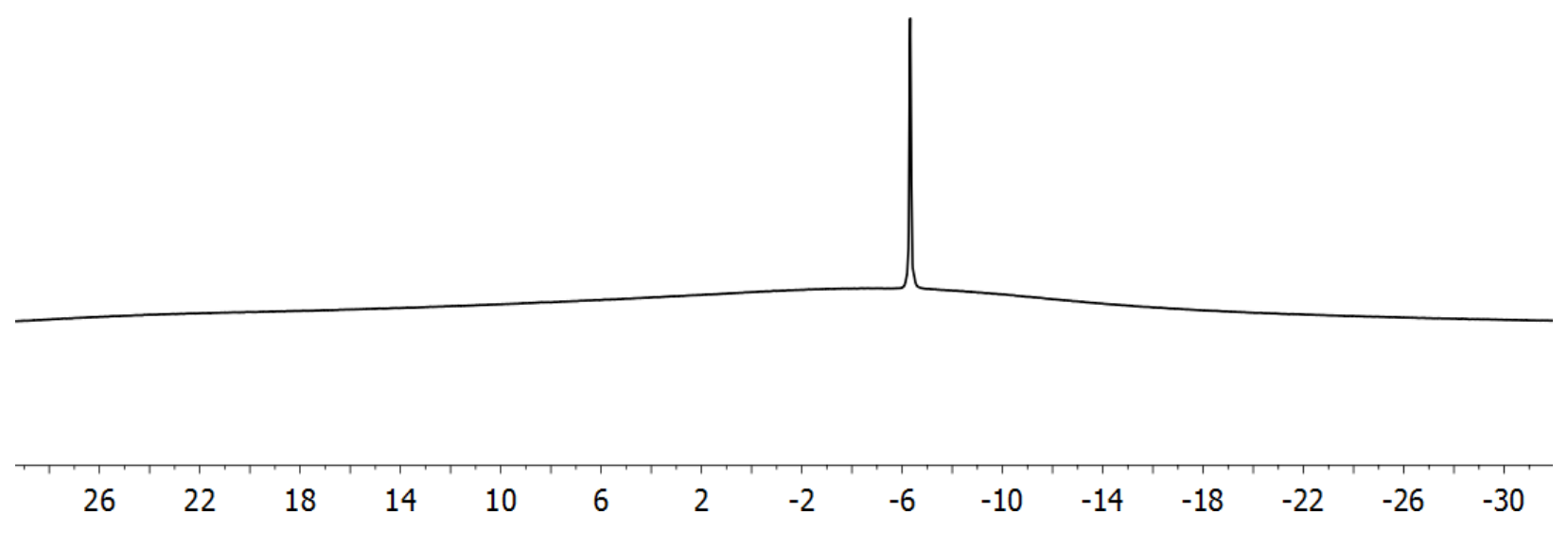

Figure S34 ${ }^{11} \mathrm{~B}$ spectrum $\left(\mathrm{CDCl}_{3}, 160 \mathrm{MHz}, 25^{\circ} \mathrm{C}\right)$ of $\left[\mathrm{Cp}^{*} \mathrm{U}(\mathrm{NTol})_{2}\left({ }^{\mathrm{t} B u}-\mathrm{Mes}^{\mathrm{PDIMe}}\right)\right]\left[\mathrm{BPh}_{4}\right]$ 


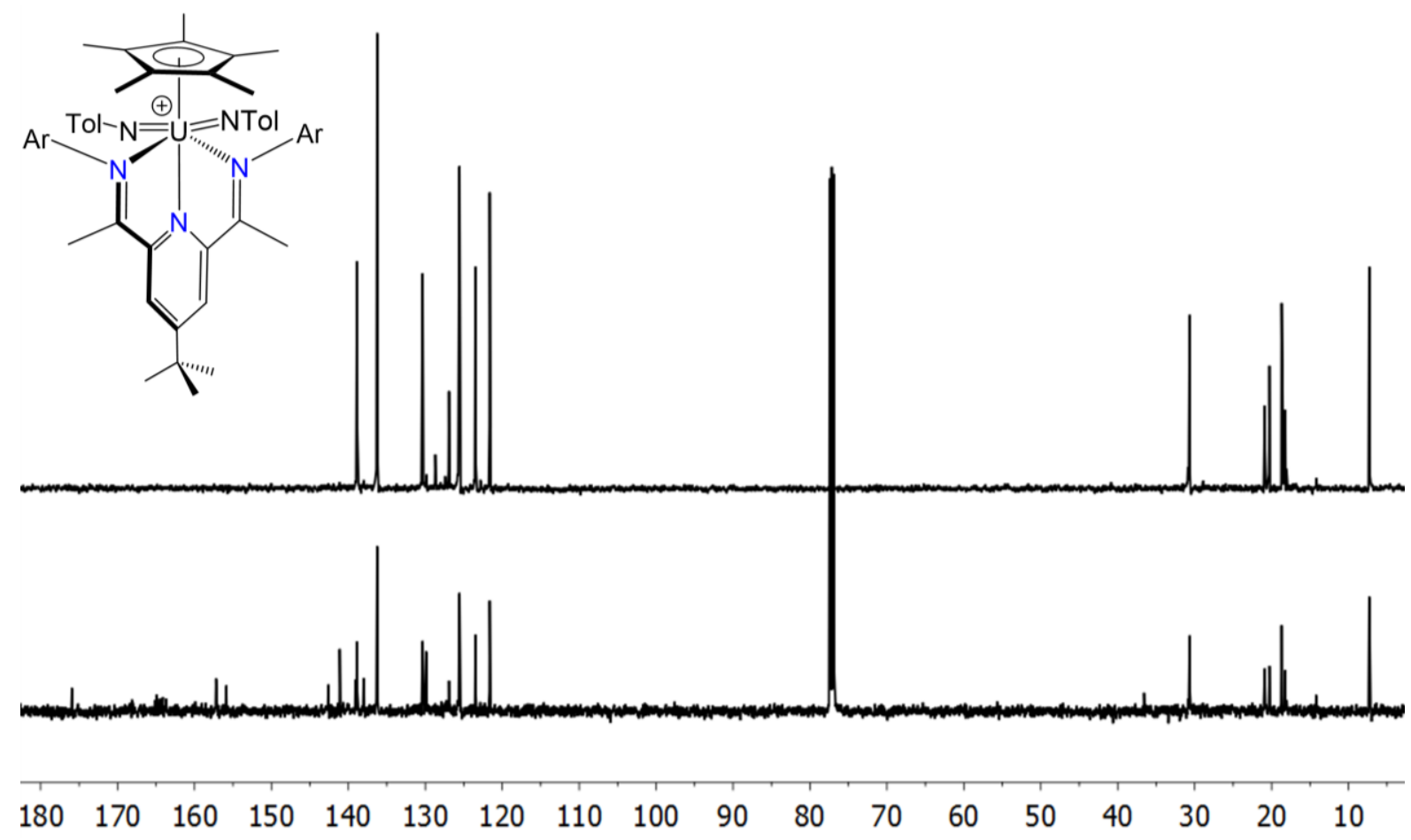

Figure $\mathrm{S} 35{ }^{13} \mathrm{C}$ and DEPT-135 spectra (in ppm) of [Cp*U(NTol) $\left.{ }_{2}\left({ }^{t} \mathrm{Bu}-{ }^{-{ }^{*}}{ }^{2} \mathrm{PDI}^{\mathrm{Me}}\right)\right]\left[\mathrm{BPh}_{4}\right]$ recorded in $\mathrm{CDCl}_{3}$ at ambient temperature 

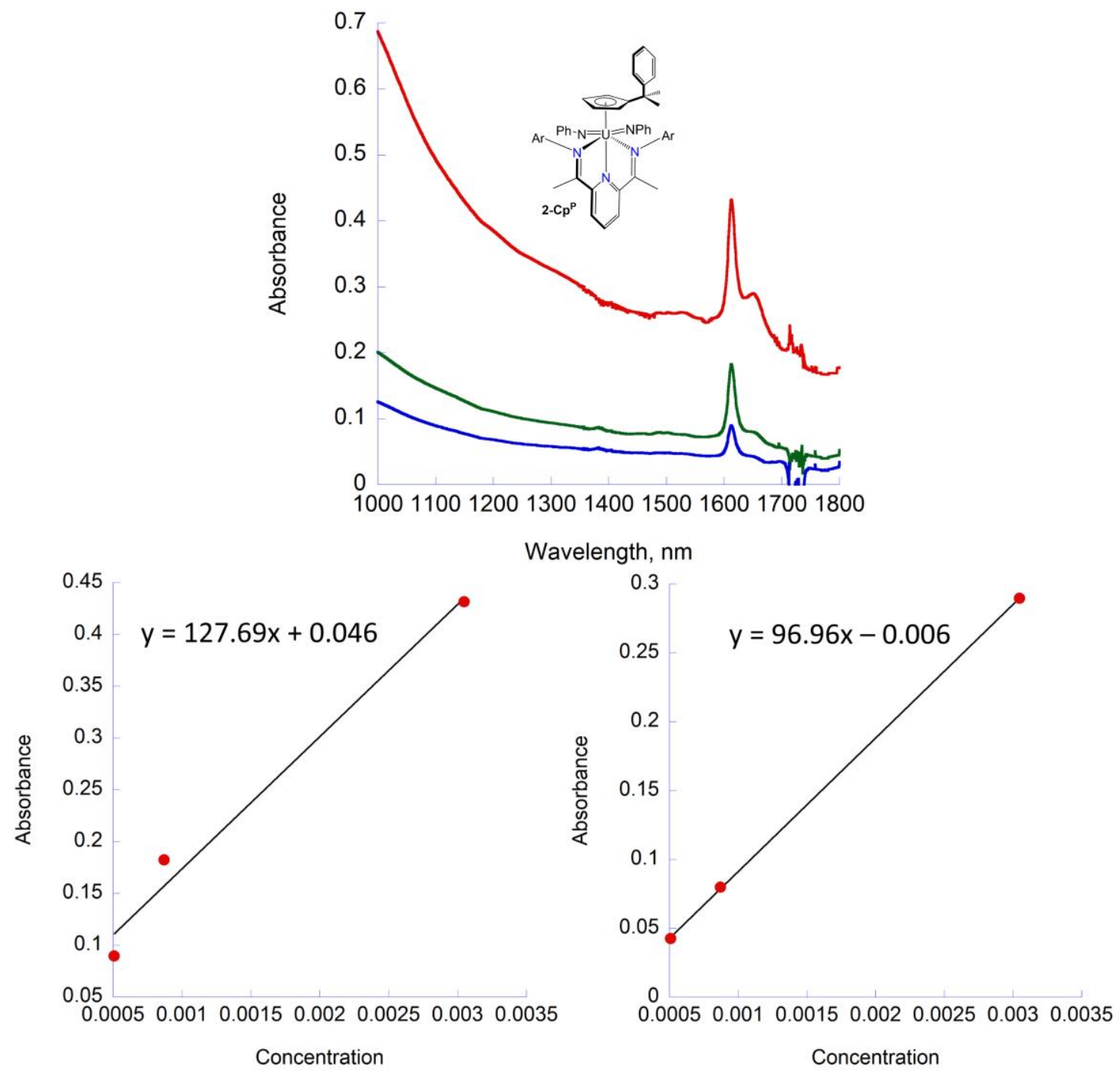

Figure S36 Top: Electronic absorption spectra of $\mathbf{2}-\mathbf{C p}^{\mathbf{P}}$ recorded in THF at ambient temperature at three separate concentrations. Bottom: Beer's Law analysis for determination of the extinction coefficient of the higher (left) and lower (right) energy near-infrared transitions. 


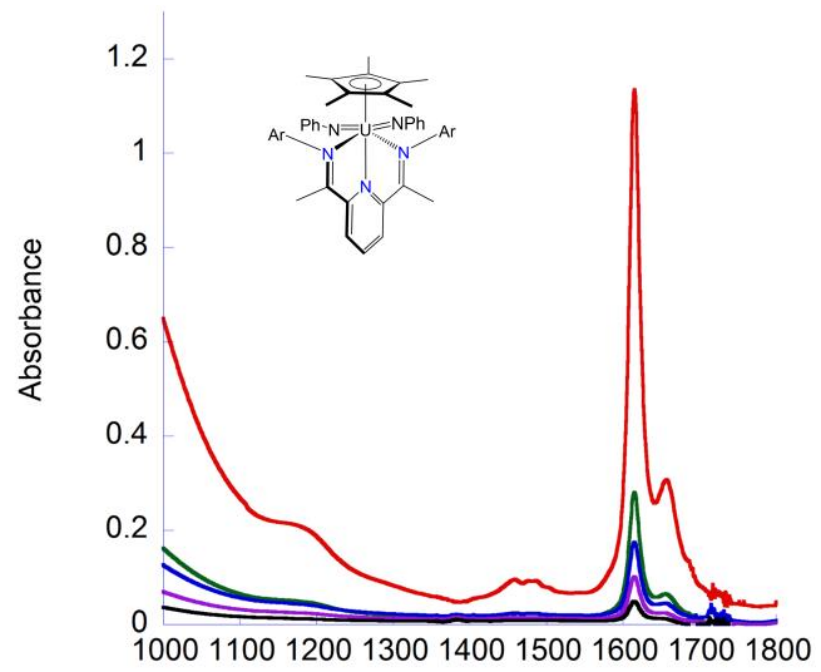

Wavelength, $\mathrm{nm}$
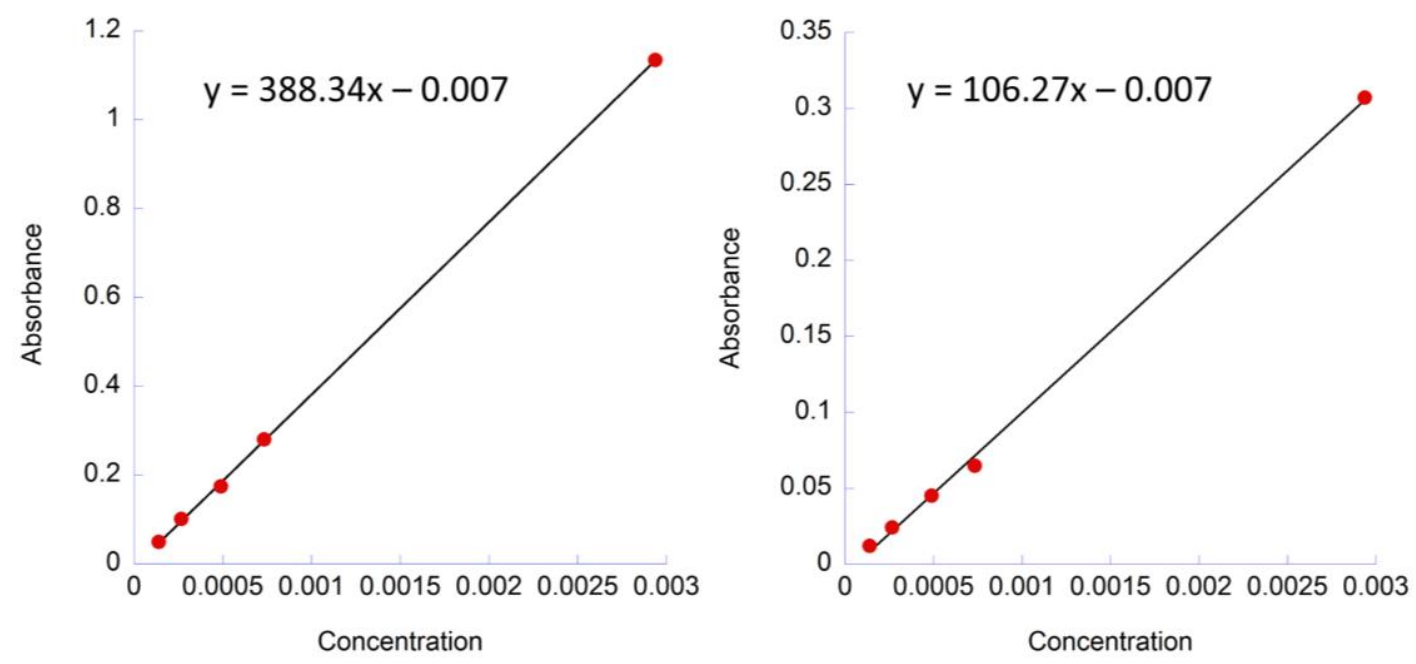

Figure S37 Top: Electronic absorption spectra of 2-Cp* recorded in THF at ambient temperature at five separate concentrations. Bottom: Beer's Law analysis for determination of the extinction coefficient of the higher (left) and lower (right) energy near-infrared transitions. 


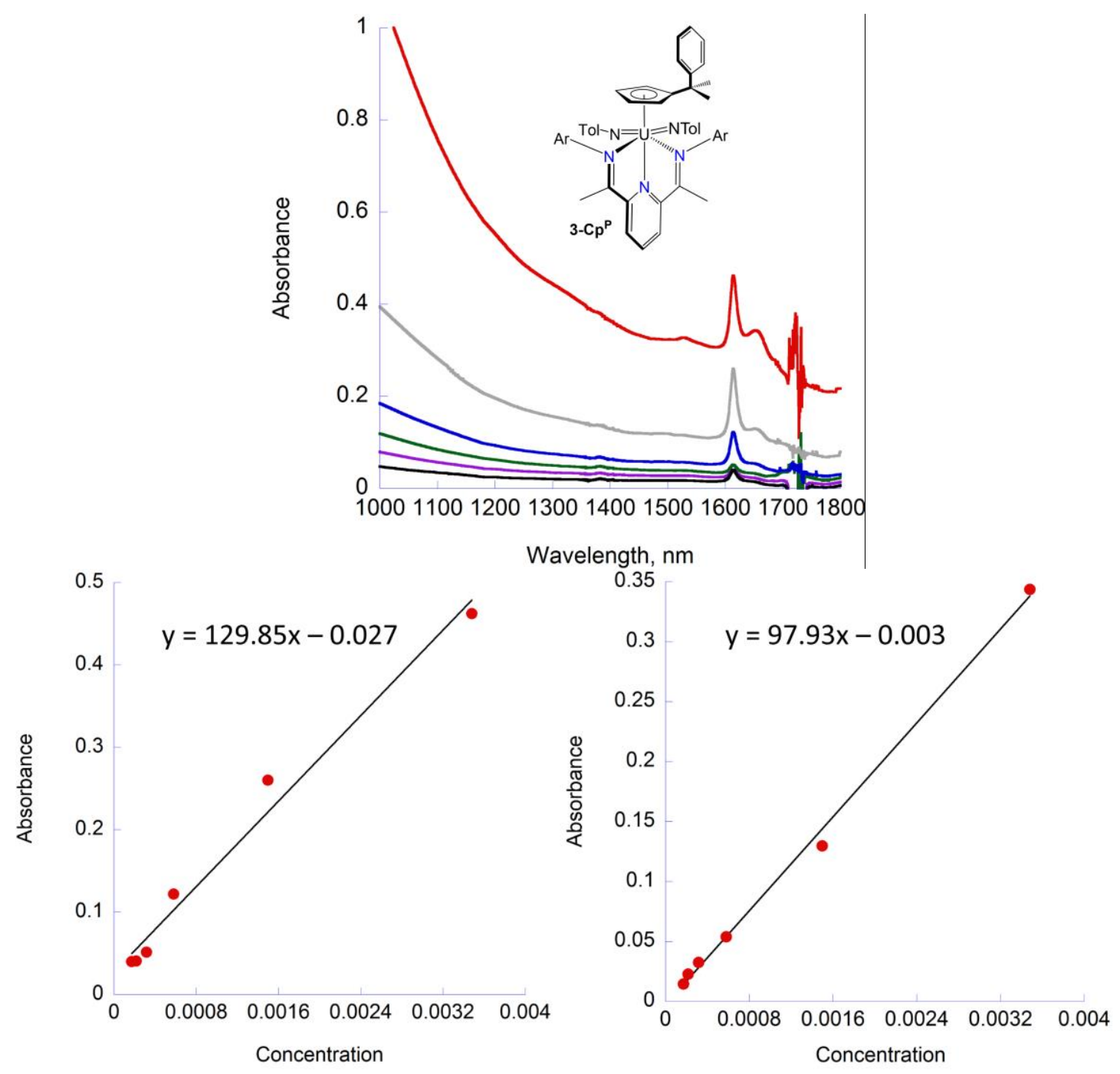

Figure S38 Top: Electronic absorption spectra of 3-Cp ${ }^{\mathbf{P}}$ recorded in THF at ambient temperature at six separate concentrations. Bottom: Beer's Law analysis for determination of the extinction coefficient of the higher (left) and lower (right) energy near-infrared transitions. 


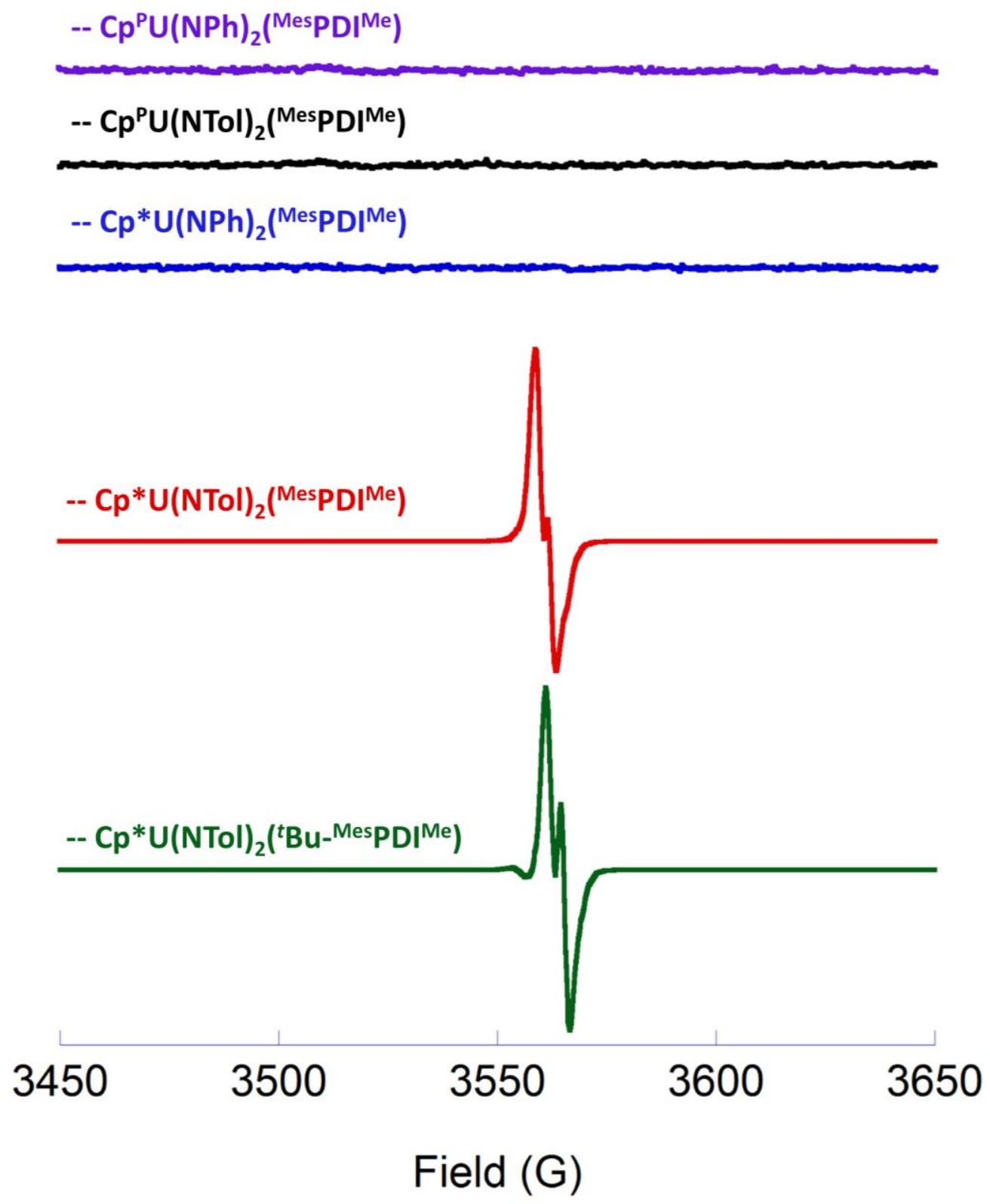

Figure S39 EPR spectra of 2-Cp $\mathbf{p}^{\mathbf{P}}$ (purple, toluene, $15.6 \mathrm{mM}$, power: $5.02 \mathrm{~mW}$, modulation: $0.15 \mathrm{mT} / 100 \mathrm{kHz}$ ), 3-CpP (black, toluene, $15.5 \mathrm{mM}$, power: $2.00 \mathrm{~mW}$, modulation: $0.15 \mathrm{mT}$ / $100 \mathrm{kHz}$ ), 2-Cp* (blue, toluene, $16.8 \mathrm{mM}$, power: $5.02 \mathrm{~mW}$, modulation: $0.15 \mathrm{mT} / 100$ $\mathrm{kHz}$ ), 3-Cp* (red, methylcyclohexane, $16.3 \mathrm{mM}$, power: $1.00 \mathrm{~mW}$, modulation: $0.25 \mathrm{mT} /$ $100 \mathrm{kHz}$ ), and 3- ${ }^{\mathrm{t}} \mathrm{Bu}$ (green, methylcyclohexane, $16.0 \mathrm{mM}$, power: $5.02 \mathrm{~mW}$, modulation: $0.15 \mathrm{mT} / 100 \mathrm{kHz}$ ) recorded at ambient temperature. Frequency: $9.85 \mathrm{GHz}$. Spectra for 2$\mathbf{C} \mathbf{p}^{\mathbf{P}}, \mathbf{3}-\mathbf{C} \mathbf{p}^{\mathbf{P}}$, and $\mathbf{2}-\mathbf{C} \mathbf{p}^{*}$ are background subtracted while spectra for $\mathbf{3}-\mathbf{C} \mathbf{p}^{*}$ and $\mathbf{3}-{ }^{-} \mathbf{B u}$ are not. 


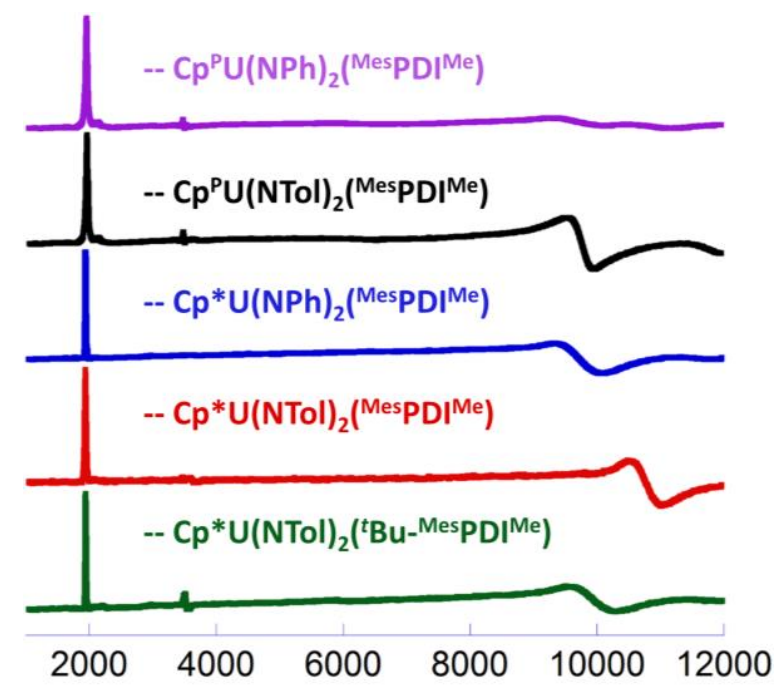

Field (G)

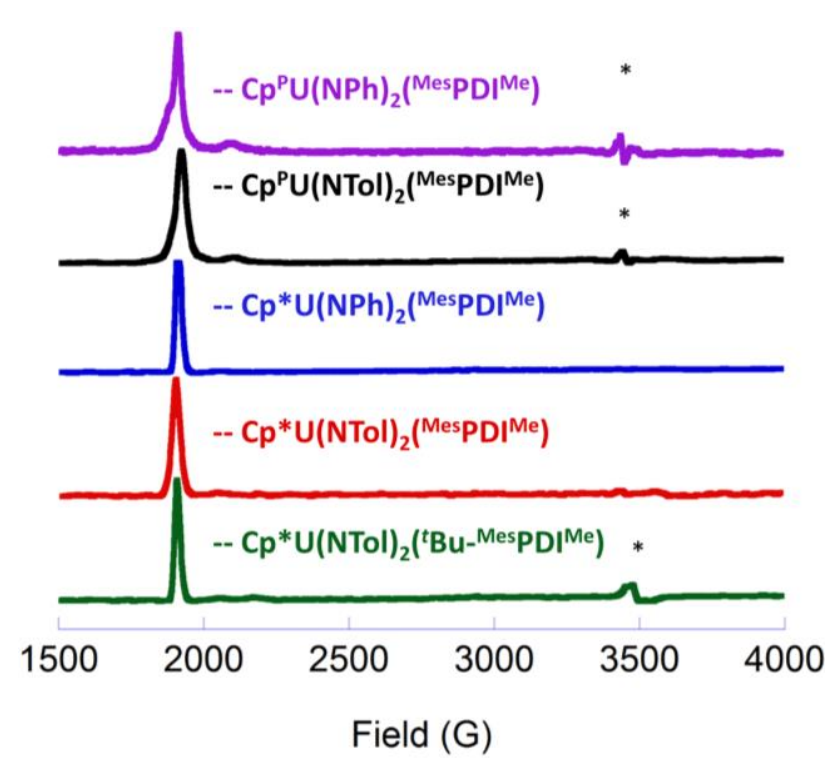

Field (G)

Figure S40 EPR spectra 2-CpP (purple, 2-MeTHF, $21.7 \mathrm{mM}$, power: $1.00 \mathrm{~mW}$, modulation: $0.4 \mathrm{mT} / 100 \mathrm{kHz}$ ), 3-CpP (black, 2-MeTHF, $12.6 \mathrm{mM}$, power: $0.10 \mathrm{~mW}$, modulation: $0.4 \mathrm{mT}$ / $100 \mathrm{kHz}$ ), 2-Cp* (blue, 2-MeTHF, $11.5 \mathrm{mM}$, power: $5.02 \mathrm{~mW}$, modulation: $0.4 \mathrm{mT} / 100$ $\mathrm{kHz}$ ), 3-Cp* (red, methylcyclohexane, $26.4 \mathrm{mM}$, power: $1.00 \mathrm{~mW}$, modulation: $0.4 \mathrm{mT} / 100$ $\mathrm{kHz}$ ), and 3-t $\mathrm{Bu}$ (green, 2-MeTHF, $19.3 \mathrm{mM}$, power: $0.10 \mathrm{~mW}$, modulation: $0.4 \mathrm{mT} / 100$ $\mathrm{kHz}$ ) recorded at $6 \mathrm{~K}$. Frequency: $9.6 \mathrm{GHz}$. Spectra are background subtracted. Asterisks at right denote radical impurities not associated with the compounds.

\begin{tabular}{|c|c|}
\hline $\mathrm{Cp}^{\mathrm{PU}}(\mathrm{NPh})_{2}$ (MesPDIMe $^{\left(\mathrm{M}^{\mathrm{M}}\right)}$ & $g_{x}=3.615, g_{y}=0.696, g_{z}=0.615$ \\
\hline $\mathrm{Cp}^{\mathrm{P} U}(\mathrm{NTol})_{2}$ (MesPDIMe $^{\mathrm{Me}}$ & $g_{x}=3.601, g_{y}=0.706, g_{z}=0.573$ \\
\hline Cp*U(NPh) $)_{2}$ (MesPDIMe): & $g_{x}=3.622, g_{y}=0.713, g_{z}=0.582$ \\
\hline Cp*U(NTol) ${ }_{2}$ (MesPDIMe): & $g_{x}=3.638, g_{y}=0.638, g_{z}=0.519$ \\
\hline $\mathrm{Cp} * \mathrm{U}(\mathrm{NTol})_{2}\left({ }^{t} \mathrm{Bu}-\mathrm{Mes} P D I^{\mathrm{Me}}\right):$ & $g_{x}=3.625, g_{y}=0.691, g_{z}=0.567$ \\
\hline
\end{tabular}




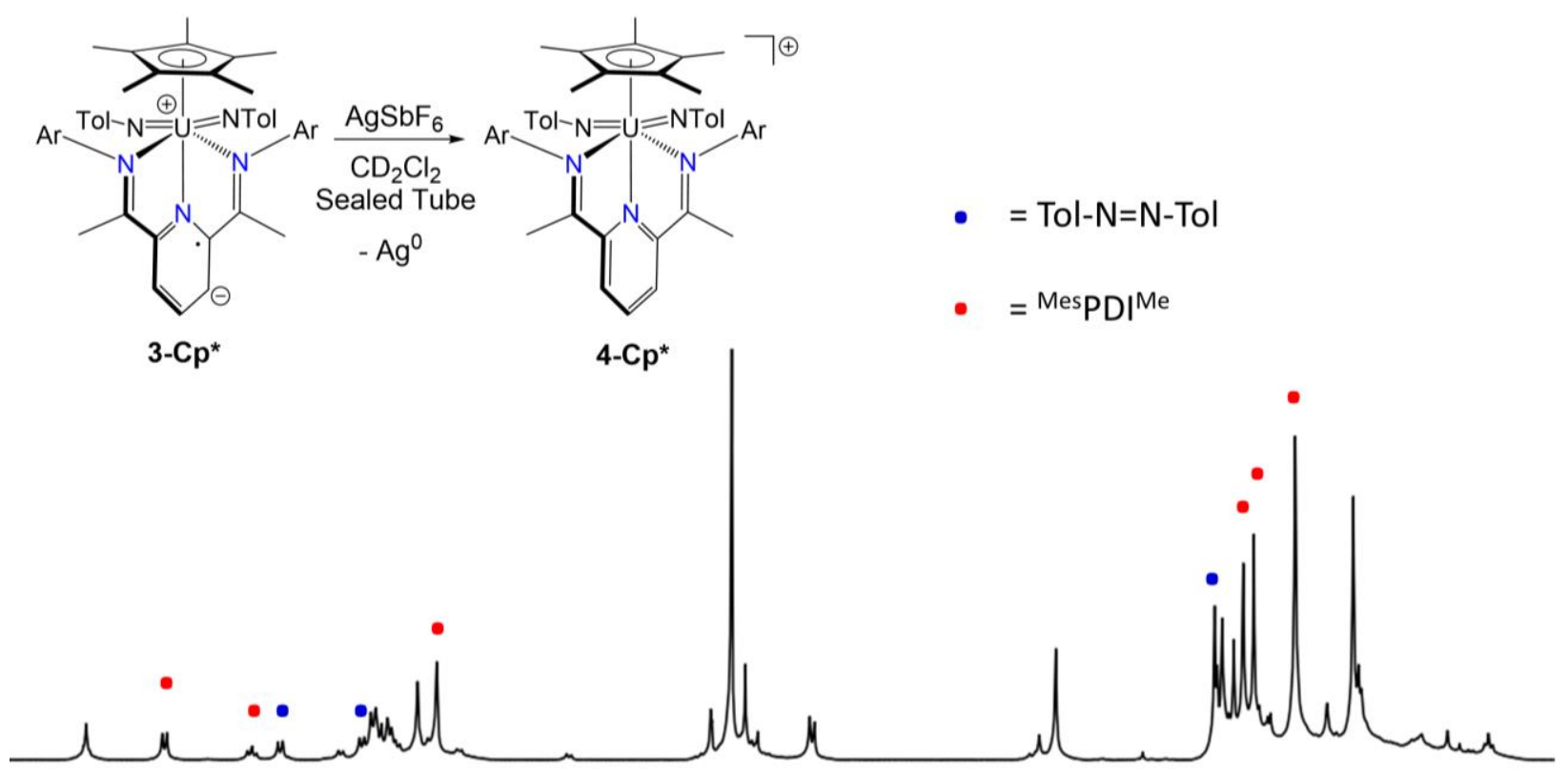

$\begin{array}{llllllllllllllllll}9.0 & 8.5 & 8.0 & 7.5 & 7.0 & 6.5 & 6.0 & 5.5 & \begin{array}{c}5.0 \\ \mathrm{ppm}\end{array} & 4.5 & 4.0 & 3.5 & 3.0 & 2.5 & 2.0 & 1.5 & 1.0 & 0 .\end{array}$

Figure $\mathrm{S} 41{ }^{1} \mathrm{H}$ NMR spectrum $\left(\mathrm{CD}_{2} \mathrm{Cl}_{2}\right.$, ambient temperature $)$ of the crude reaction mixture derived from addition of $\mathrm{AgSbF}_{6}$ to $3-\mathrm{Cp}^{*}$. The tube was sealed prior to mixing of the solid $\mathrm{AgSbF}_{6}$ with the solution of $\mathbf{3}-\mathbf{C p}^{*}$, then shaken for twenty minutes prior to obtaining the spectrum. Identified byproducts, di-para-tolyldiazene and MesPDIMe, are labeled. Dihydrogen is not observed. Silver plating on the inside of the NMR tube suggested reduction of silver(I). 


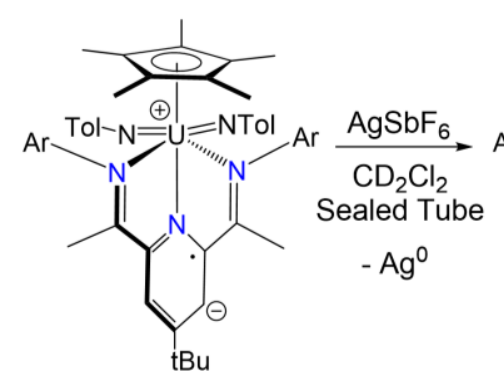

3- ${ }^{t} \mathrm{Bu}$

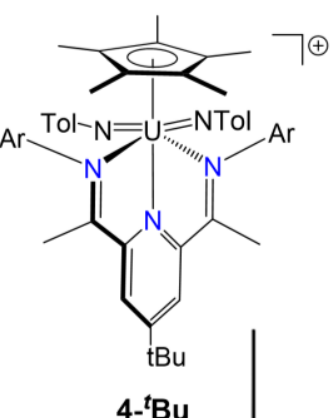

4-t ${ }^{t} \mathrm{Bu}$
- $\quad$ Tol-N=N-Tol

- $={ }^{t} \mathrm{Bu}-{ }^{\mathrm{Mes}} \mathrm{PDI} \mathrm{Me}^{\mathrm{Me}}$

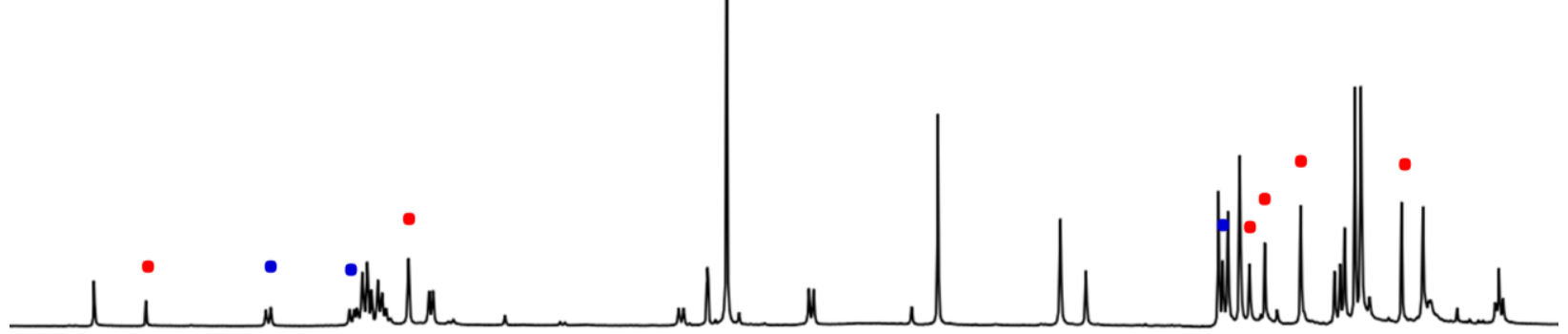

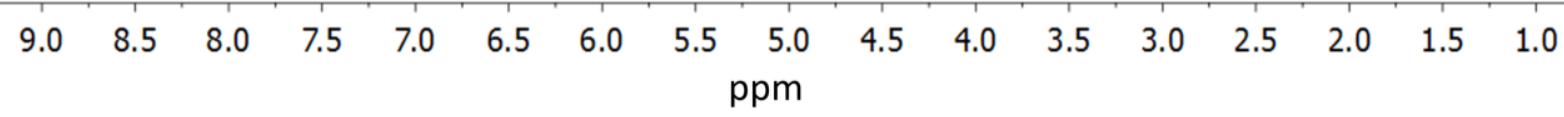

Figure $\mathrm{S} 42{ }^{1} \mathrm{H}$ NMR spectrum $\left(\mathrm{CD}_{2} \mathrm{Cl}_{2}\right.$, ambient temperature) of the crude reaction mixture derived from addition of $\mathrm{AgSbF}_{6}$ to $3 \mathbf{- t}^{-t} \mathbf{B u}$. The tube was sealed prior to mixing of the solid $\mathrm{AgSbF}_{6}$ with the solution of $\mathbf{3}^{-}{ }^{-} \mathbf{B u}$, then shaken for twenty minutes prior to obtaining the spectrum. Identified byproducts, di-para-tolyldiazene and ${ }^{t} \mathrm{Bu}$-MesPDIMe, are labeled. Dihydrogen is not observed. Silver plating on the inside of the NMR tube suggested reduction of silver(I). 


\section{X-ray Structural Determination}

General Procedures. Data were collected using either a Nonius Kappa CCD diffractometer with Mo-K $\alpha$ radiation $(\lambda=0.71073 \AA$ ) at Purdue University, a Rigaku Rapid II curved image plate diffractometer with $\mathrm{Cu}-\mathrm{K} \alpha(\lambda=1.54178 \AA)$ at Purdue University, or a Bruker AXS D8

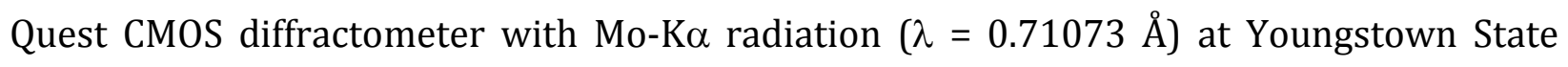
University. The KappaCCD instrument features a fine focus sealed tube X-ray source with graphite monochromator. The Rigaku Rapid II diffractometer and the Bruker CMOS diffractometer instrument are equipped with an X-ray microsources with a laterally graded multilayers (Goebel) mirror for monochromatization. Single crystals were mounted on Mitegen micromesh or loop mounts using a trace of mineral oil and cooled in-situ to $200(2), 150(2)$ or 100(2) K for data collection. Data on the KappaCCD instrument were collected using the Nonius Collect software [A]. Data obtained from the Rigaku Rapid II instrument were collected using the dtrek option of CrystalClear [B]. Both kinds of data sets were processed using HKL3000 [C] and data were corrected for absorption and scaled using Scalepack [C]. Data of the Bruker CMOS instrument were collected, reflections were indexed and processed, and the files scaled and corrected for absorption using APEX2 [D]. The space groups were assigned and the structures were solved by direct methods using XPREP within the SHELXTL suite of programs [E] and refined by full matrix least squares against $F^{2}$ with all reflections using Shelxl2013 or 2014 [F] using the graphical interface Shelxle [G]. If not specified otherwise, $\mathrm{H}$ atoms attached to carbon atoms were positioned geometrically and constrained to ride on their parent atoms, with carbon hydrogen bond distances of $0.95 \AA$ for and aromatic $\mathrm{C}-\mathrm{H}, 1.00$, and $0.98 \AA$ for aliphatic $\mathrm{C}-\mathrm{H}$ and $\mathrm{CH}_{3}$ moieties, respectively. Methyl $\mathrm{H}$ atoms were allowed to rotate but not to tip to best fit the experimental electron density. $U_{\text {iso }}(H)$ values were set to a multiple of $U_{\text {eq }}(C)$ with 1.5 for $\mathrm{CH}_{3}$, and 1.2 for $\mathrm{C}-\mathrm{H}$ units, respectively. Non merohedric twinning was handled using the programs Rotax $[\mathrm{H}]$ and WinGX $[\mathrm{I}]$.

Complete crystallographic data, in CIF format, have been deposited with the Cambridge Crystallographic Data Centre. These data can be obtained free of charge from The Cambridge Crystallographic Data Centre via www.ccdc.cam.ac.uk/data_request/cif. 
The Bruker CMOS X-ray diffractometer was funded by NSF Grant DMR 1337296.

A) Nonius (1998). Collect Users Manual, Nonius Delft, The Netherlands.

B) Rigaku (2014). CrystalClear-SM Expert 2.1 b32, Rigaku Corp., The Woodlands, Texas, USA.

C) Otwinowski Z. \& Minor, W. (1997). Methods Enzymol., 276, 307-327

D) Apex2 v2013.4-1, v2014.11, v2014.1-1, Saint V8.34A, SAINT V8.30C, Bruker AXS Inc.: Madison (WI), USA, 2013/2014.

E) a) SHELXTL (Version 6.14) (2000-2003) Bruker Advanced X-ray Solutions, Bruker AXS Inc., Madison, Wisconsin: USA. b) Sheldrick, G. M. (2008). Acta Cryst. A64, 112-122.

F) a) Sheldrick, G. M. (2015). Acta Cryst. C71, 3-8. b) Sheldrick, G. M. (2013). University of Göttingen, Germany.

G) SHELXLE various Revisions (Hübschle et al., 2011), SHELXLE. Hübschle, C. B., Sheldrick, G. M. and Dittrich, B. (2011). J. Appl. Cryst., 44, 1281--1284.

H) Cooper, R. I., Gould, R. O., Parsons, S, Watkin, D. J. (2002). J. Appl. Cryst. 35, 168-174.

I) Farrugia, L. J. (2012). J. Appl. Cryst. 45, 849-854. 


\section{Crystallographic Details 1-Cp ${ }^{\mathbf{P}}$}

Complex: $\left.\left[\mathrm{Cp}^{\mathrm{P}}{ }^{\mathrm{Mes}}{ }^{\mathrm{PDI}} \mathrm{I}^{\mathrm{Me}}\right)\right]_{2}$

Local name: jk955

CCDC number: 1457425

Table S1 Experimental parameters of 1-Cp ${ }^{\mathbf{P}}$.

\begin{tabular}{|c|c|}
\hline \multicolumn{2}{|l|}{ Crystal data } \\
\hline Chemical formula & $\mathrm{C}_{82} \mathrm{H}_{92} \mathrm{~N}_{6} \mathrm{U}_{2} \cdot \mathrm{C}_{62} \mathrm{H}_{78} \mathrm{~N}_{6} \mathrm{O}_{2} \mathrm{U}_{2} \cdot 8\left(\mathrm{C}_{4} \mathrm{H}_{8} \mathrm{O}\right)$ \\
\hline$M_{\mathrm{r}}$ & 3629.86 \\
\hline Crystal system, space group & Monoclinic, $P 2_{1} / c$ \\
\hline Temperature $(\mathrm{K})$ & 100 \\
\hline$a, b, c(\AA)$ & $15.803(2), 15.356(2), 32.896(2)$ \\
\hline$\beta\left(^{\circ}\right)$ & $103.472(4)$ \\
\hline$V\left(\AA^{3}\right)$ & $7763.2(15)$ \\
\hline$Z$ & 2 \\
\hline Radiation type & $\mathrm{Cu} K \alpha$ \\
\hline$\mu\left(\mathrm{mm}^{-1}\right)$ & 12.09 \\
\hline Crystal size $(\mathrm{mm})$ & $0.20 \times 0.20 \times 0.03$ \\
\hline \multicolumn{2}{|l|}{ Data collection } \\
\hline Diffractometer & Rigaku Rapid II curved image plate diffractometer \\
\hline Absorption correction & Multi-scan SCALEPACK (Otwinowski \& Minor, 1997) \\
\hline$T_{\min }, T_{\max }$ & $0.206,0.713$ \\
\hline $\begin{array}{l}\text { No. of measured, independent and } \\
\text { observed }[I>2 \sigma(I)] \text { reflections }\end{array}$ & $101324,101324,79003$ \\
\hline$(\sin \theta / \lambda)_{\max }\left(\AA^{-1}\right)$ & 0.610 \\
\hline \multicolumn{2}{|l|}{ Refinement } \\
\hline$R\left[F^{2}>2 \sigma\left(F^{2}\right)\right], w R\left(F^{2}\right), S$ & $0.102,0.286,1.03$ \\
\hline No. of reflections & 101324 \\
\hline No. of parameters & 1160 \\
\hline No. of restraints & 2664 \\
\hline \multirow[t]{2}{*}{ H-atom treatment } & $\mathrm{H}$-atom parameters constrained \\
\hline & $\begin{array}{l}w=1 /\left[\sigma^{2}\left(F_{0}^{2}\right)+(0.114 P)^{2}+636.631 P\right] \\
\text { where } P=\left(F_{0}^{2}+2 F_{c}^{2}\right) / 3\end{array}$ \\
\hline$\Delta \rho_{\max }, \Delta \rho_{\min }\left(\mathrm{e} \AA^{-3}\right)$ & $7.53,-4.60$ \\
\hline
\end{tabular}




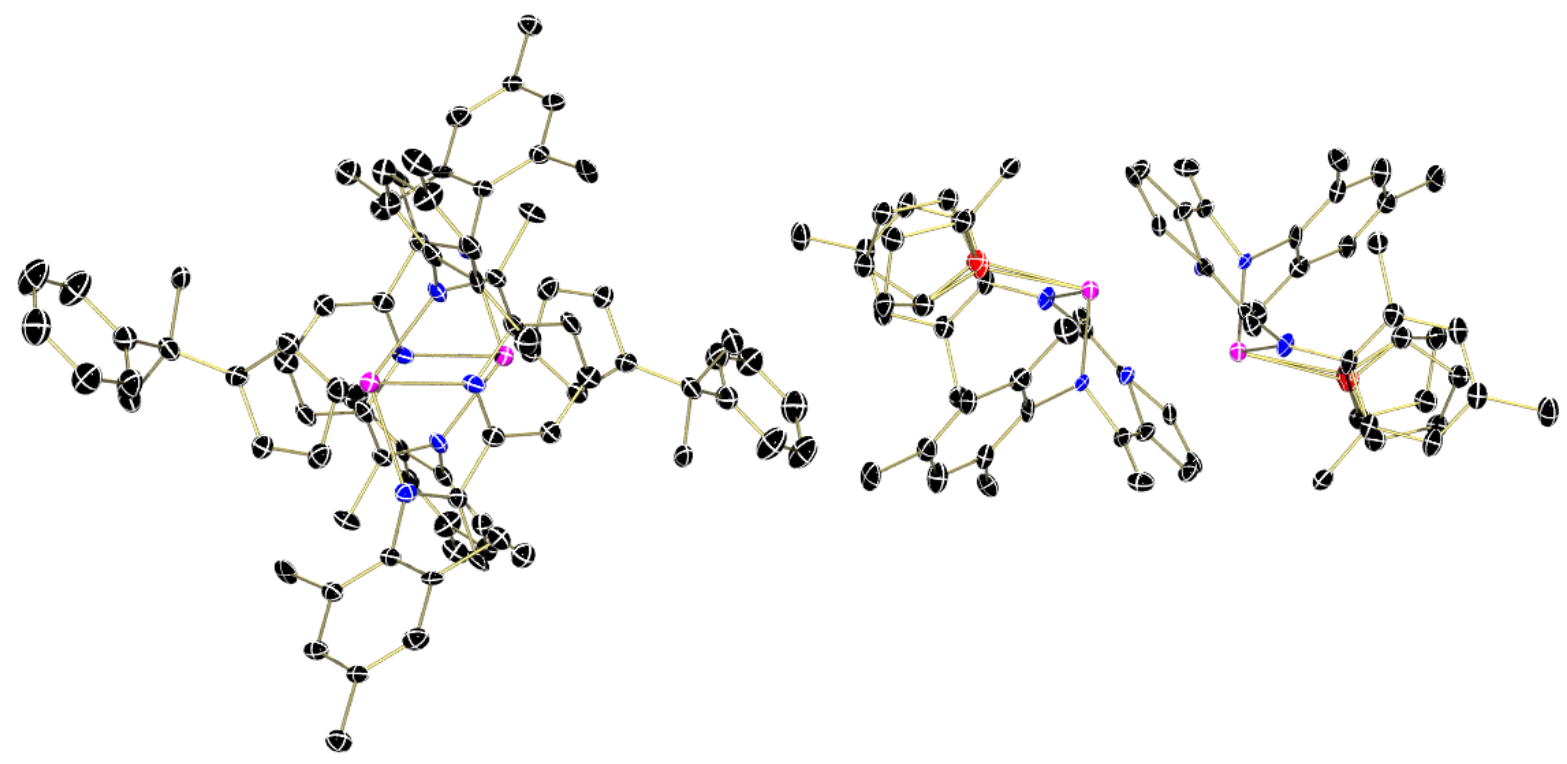

Figure S43 Cocrystallized molecules of [Cp $\left.{ }^{\mathrm{P} U}\left(\mathrm{MesPDI}^{\mathrm{Me}}\right)\right]_{2}$ and $\left[\left(\mathrm{MesPDI}^{\mathrm{Me}}\right) \mathrm{U}(\mathrm{THF})\right]_{2}$ displayed at $30 \%$ probability ellipsoids. Cocrystallized THF molecules and hydrogen atoms have been omitted for clarity.

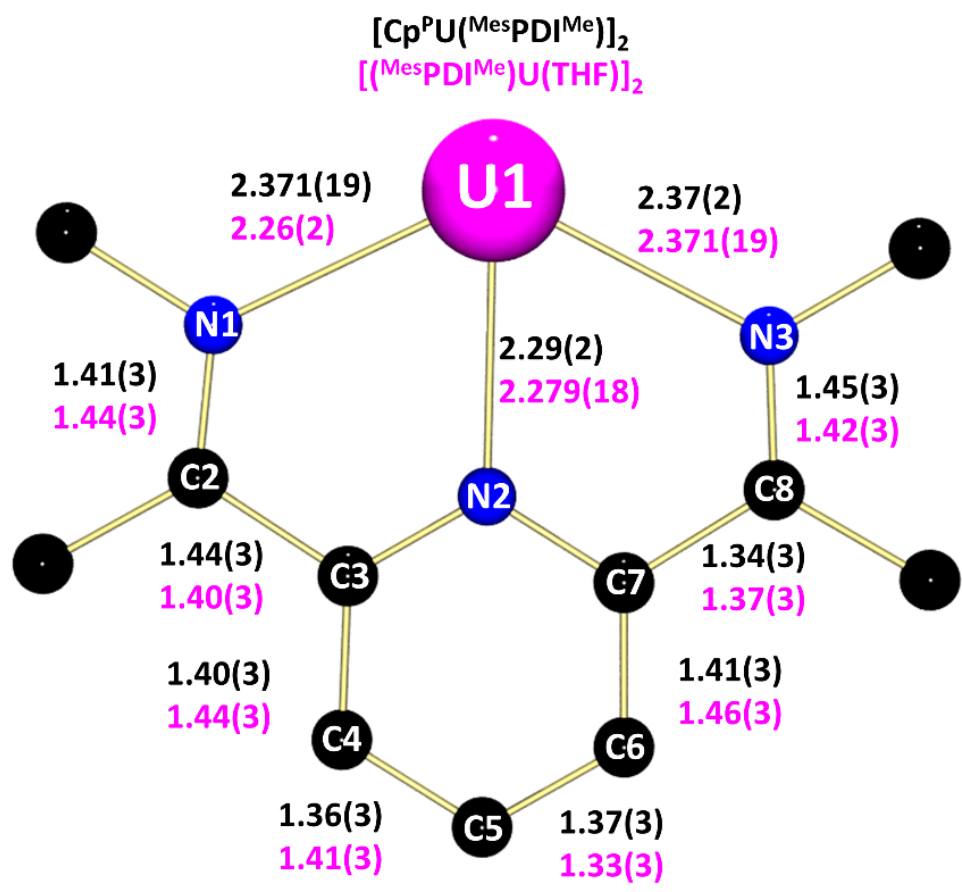

Figure S44 Bond length comparison $(\AA)$ of pyridine(diimine) ligands in cocrystallized $\left[\mathrm{Cp}^{\mathrm{P} U}\left({ }^{\mathrm{Mes} P D I} \mathrm{Ie}^{\mathrm{Me}}\right)\right]_{2}$ (black) and [(MesPDIMe)U(THF) $]_{2}$ (magenta). 


\section{Refinement details:}

The crystal under investigation was found to be non-merohedrically twinned. HKL3000 lacks the ability to simultaneously integrate more than one twin domain. Rigaku programs compatible with the diffractometer (twinsolve) gave unsatisfactory results. With no usable data set obtainable through simultaneous integration of both twin domains, the data were instead handled as if not twinned, with only the major domain integrated, and converted into an hklf 5 type format hkl file after integration using the "Make HKLF5 File" routine as implemented in WinGX. The twin law matrix was obtained using the program ROTAX as implemented in WinGX and corresponds to a $180^{\circ}$ rotation around either the a or c axis, the twin matrix used was

$$
\begin{aligned}
& {\left[\begin{array}{rrr}
1.000 & 0.000 & 0.000
\end{array}\right]} \\
& {\left[\begin{array}{ccc}
0.000 & -1.000 & 0.000
\end{array}\right]} \\
& {\left[\begin{array}{ccc}
-0.970 & 0.000 & -1.000
\end{array}\right]}
\end{aligned}
$$

The Overlap R1 and R2 values in the "Make HKLF5 File" routine used were 0.18, i.e. reflections with a discriminator function less or equal to overlap radius of 0.18 were counted overlapped, all others as single. The discriminator function used was the "delta function on index non-integrality". No reflections were omitted.

The structure was solved using direct methods with all reflections of component 1 . The structure was refined using the hklf 5 routine with all reflections of component 1 (including the overlapping ones) as obtained from WinGX, resulting in a BASF value of 0.332(2). No $\mathrm{R}_{\mathrm{int}}$ value is obtainable for the hklf 5 type file using the WinGX routine.

One metal coordinated THF molecule and four interstitial THF molecules were refined as disordered over each two orientations. All THF moieties were refined to have similar geometries (SAME command in Shelxl), equivalent $\mathrm{O}-\mathrm{C}$ bond distances as well as all C-C bond distances were restrained to be similar. In the least well defined pair of THF molecules (05, C93-C96 and 05B, C93B-C96B) selected distances between carbon atoms of the two moieties were restrained to be at least three Ångstroms (C95B to C90B, C95B to C90, C94B to C90B, C94B to C90, C95B to C89B, C95B to C89, C94B to C89B, C94B to C89). The Uij components of the ADPs of disordered pairs of molecules were restrained to be similar if closer than 1.7 Angstroms (SIMU command in Shelxl). A weak anti-bumping restraint was applied for all atoms (BUMP -0.02 restraint in Shelxl). Subject to these conditions the occupancy ratios refined to $0.78(3)$ to $0.22(3)$ for the THF molecule of 01 , to $0.83(3)$ to $0.17(3)$ for that of 02 , to $0.76(3)$ to $0.24(3)$ for that of 03 , to $0.76(6)$ to $0.24(6)$ for that of 04 , and to $0.85(3)$ to $0.15(3)$ for that of 05 . 


\section{Crystallographic Details 3-Cp*}

\section{Complex: $\mathrm{Cp}^{*} \mathrm{U}(\mathrm{NTol})_{2}\left({ }^{\mathrm{Mes} P D I}{ }^{\mathrm{Me}}\right)$}

Local name: jk374

CCDC number: 1457420

Table S2 Experimental parameters of 3-Cp*.

\begin{tabular}{|c|c|}
\hline \multicolumn{2}{|l|}{ Crystal data } \\
\hline Chemical formula & $\mathrm{C}_{51} \mathrm{H}_{60} \mathrm{~N}_{5} \mathrm{U} \cdot \mathrm{C}_{7} \mathrm{H}_{8}$ \\
\hline$M_{\mathrm{r}}$ & 1073.20 \\
\hline Crystal system, space group & Monoclinic, $C 2 / c$ \\
\hline Temperature (K) & 150 \\
\hline$a, b, c(\AA)$ & $26.752(3), 11.2910(9), 17.8109(9)$ \\
\hline$\beta\left({ }^{\circ}\right)$ & $111.537(5)$ \\
\hline$V\left(\AA^{3}\right)$ & $5004.3(7)$ \\
\hline$Z$ & 4 \\
\hline Radiation type & Mo $K \alpha$ \\
\hline$\mu\left(\mathrm{mm}^{-1}\right)$ & 3.29 \\
\hline Crystal size $(\mathrm{mm})$ & $0.54 \times 0.24 \times 0.24$ \\
\hline \multicolumn{2}{|l|}{ Data collection } \\
\hline Diffractometer & Nonius Kappa CCD diffractometer \\
\hline Absorption correction & Multi-scan SCALEPACK (Otwinowski \& Minor, 1997) \\
\hline$T_{\min }, T_{\max }$ & $0.390,0.455$ \\
\hline $\begin{array}{l}\text { No. of measured, independent and } \\
\text { observed }[I>2 \sigma(I)] \text { reflections }\end{array}$ & $23084,5593,4233$ \\
\hline$R_{\text {int }}$ & 0.074 \\
\hline$(\sin \theta / \lambda)_{\max }\left(\AA^{-1}\right)$ & 0.649 \\
\hline \multicolumn{2}{|l|}{ Refinement } \\
\hline$R\left[F^{2}>2 \sigma\left(F^{2}\right)\right], w R\left(F^{2}\right), S$ & $0.050,0.130,1.23$ \\
\hline No. of reflections & 5593 \\
\hline No. of parameters & 438 \\
\hline No. of restraints & 435 \\
\hline \multirow[t]{2}{*}{$\mathrm{H}$-atom treatment } & H-atom parameters constrained \\
\hline & $\begin{array}{l}w=1 /\left[\sigma^{2}\left(F_{0}^{2}\right)+(0.0269 P)^{2}+59.1497 P\right] \\
\text { where } P=\left(F_{\mathrm{o}}^{2}+2 F_{\mathrm{c}}^{2}\right) / 3\end{array}$ \\
\hline$\Delta \rho_{\max }, \Delta \rho_{\min }\left(\mathrm{e} \AA^{-3}\right)$ & $2.78,-4.08$ \\
\hline
\end{tabular}




\section{Refinement details:}

The 1,2,3,4,5-pentamethylcyclopentadienide anion is disordered across a mirror plane.

A mesitylene moiety was refined as disordered over two orientations. The two moieties were restrained to have similar geometries, and $\mathrm{U}^{\mathrm{ij}}$ components of the ADPs of their atoms were restrained to be similar if closer than 1.7 Ångstrom. One pair of ortho C atoms and one pair of methyl $\mathrm{C}$ atoms from both moieties were each constrained to have identical ADPs.

A toluene molecule is disordered around a crystallographic inversion center. The benzene ring was constrained to resemble an ideal hexagon with C-C distances of 1.39 Ångstrom. Uij components of the ADPs of its atoms were restrained to be similar if closer than 1.7 Ångstrom, and atoms were restrained to be close to isotropic. 


\section{Crystallographic Details $\mathbf{3}^{-{ }^{-} \mathbf{B u}}$}

Complex: Cp*U(NTol $)_{2}\left({ }^{t} \mathrm{Bu}-{ }^{-M e s P D I M e}\right)$

Local name: jk635

CCDC number: 1457421

Table S3 Experimental parameters of $\mathbf{3}^{-\boldsymbol{t}} \mathbf{B u}$

\begin{tabular}{|c|c|}
\hline \multicolumn{2}{|l|}{ Crystal data } \\
\hline Chemical formula & $2\left(\mathrm{C}_{55} \mathrm{H}_{68} \mathrm{~N}_{5} \mathrm{U}\right) \cdot 0.5\left(\mathrm{C}_{14} \mathrm{H}_{14} \mathrm{~N}_{2}\right) \cdot 3\left(\mathrm{C}_{5} \mathrm{H}_{12}\right)$ \\
\hline$M_{\mathrm{r}}$ & 2395.91 \\
\hline Crystal system, space group & Triclinic, $P \overline{1}$ \\
\hline Temperature $(\mathrm{K})$ & 200 \\
\hline$a, b, c(\AA)$ & $13.8851(11), 14.2035(11), 18.679(2)$ \\
\hline$\alpha, \beta, \gamma\left(^{\circ}\right)$ & $68.317(5), 86.562(9), 63.162(5)$ \\
\hline$V\left(\AA^{3}\right)$ & $3028.9(5)$ \\
\hline$Z$ & 1 \\
\hline Radiation type & $\mathrm{Cu} K \alpha$ \\
\hline$\mu\left(\mathrm{mm}^{-1}\right)$ & 7.85 \\
\hline Crystal size $(\mathrm{mm})$ & $0.20 \times 0.16 \times 0.05$ \\
\hline \multicolumn{2}{|l|}{ Data collection } \\
\hline Diffractometer & Rigaku Rapid II curved image plate diffractometer \\
\hline Absorption correction & Multi-scan SCALEPACK (Otwinowski \& Minor, 1997) \\
\hline$T_{\min }, T_{\max }$ & $0.057,0.678$ \\
\hline $\begin{array}{l}\text { No. of measured, independent and } \\
\text { observed }[I>2 \sigma(I)] \text { reflections }\end{array}$ & $10392,10392,8595$ \\
\hline$R_{\text {int }}$ & 0.061 \\
\hline$(\sin \theta / \lambda)_{\max }\left(\AA^{-1}\right)$ & 0.602 \\
\hline \multicolumn{2}{|l|}{ Refinement } \\
\hline$R\left[F^{2}>2 \sigma\left(F^{2}\right)\right], w R\left(F^{2}\right), S$ & $0.064,0.151,1.14$ \\
\hline No. of reflections & 10392 \\
\hline No. of parameters & 753 \\
\hline No. of restraints & 446 \\
\hline \multirow[t]{2}{*}{$\mathrm{H}$-atom treatment } & $\mathrm{H}$-atom parameters constrained \\
\hline & $\begin{array}{l}w=1 /\left[\sigma^{2}\left(F_{0}^{2}\right)+(0.0396 P)^{2}+21.5257 P\right] \\
\text { where } P=\left(F_{0}^{2}+2 F_{c}^{2}\right) / 3\end{array}$ \\
\hline$\Delta \rho_{\max }, \Delta \rho_{\min }\left(\mathrm{e} \AA^{-3}\right)$ & $2.31,-2.76$ \\
\hline
\end{tabular}




\section{Refinement details:}

A ditolyldiazene molecule is located atop of a crystallographic inversion center and was refined as disordered with two pentane molecules. The aromatic ring was constrained to resemble an ideal hexagon with C-C bonds of 1.39 Ångstrom. The C-C bond distances of the pentane molecules as well as another ill-defined, not disordered, pentane molecule were restrained to expected values: 1.5 for C-C(methyl) and 1.45 Ångstroms for all other C-C bonds. Subject to these conditions the occupancy ratio refined to 0.501(13) to 0.499(13) in favor of pentane. The not disordered pentane molecule was also subjected to a rigid bond restraint (RIGU in Shelxl), and C atoms were restrained to be approximately isotropic.

The tert butyl group is rotationally disordered over two positions. The two moieties were restrained to have similar geometries. Atoms were restrained to be close to isotropic and Uij components of ADPs were restrained to be similar if closer than 1.7 Ångstrom. 


\section{Crystallographic Details 4-Cp*}

\section{Complex: [Cp*U(NTol $\left.)_{2}\left(\mathrm{MesPDI}^{\mathrm{Me}}\right)\right]\left[\mathrm{SbF}_{6}\right]$}

Local name: jk8175

\section{CCDC number: 1457424}

\section{Table S4 Experimental parameters of 4-Cp*}

\begin{tabular}{|c|c|}
\hline \multicolumn{2}{|l|}{ Crystal data } \\
\hline Chemical formula & $\mathrm{C}_{51} \mathrm{H}_{60} \mathrm{~N}_{5} \mathrm{U} \cdot \mathrm{F}_{6} \mathrm{Sb} \cdot \mathrm{C}_{4} \mathrm{H}_{8} \mathrm{O}$ \\
\hline$M_{\mathrm{r}}$ & 1288.86 \\
\hline Crystal system, space group & Monoclinic, $C 2 / c$ \\
\hline Temperature $(\mathrm{K})$ & 100 \\
\hline$a, b, c(\AA)$ & 44.3345 (19), 16.1486 (7), 15.2487 (7) \\
\hline$\beta\left(^{\circ}\right)$ & $105.7359(14)$ \\
\hline$V\left(\AA^{3}\right)$ & $10508.0(8)$ \\
\hline$Z$ & 8 \\
\hline Radiation type & Mo $K \alpha$ \\
\hline$\mu\left(\mathrm{mm}^{-1}\right)$ & 3.66 \\
\hline Crystal size $(\mathrm{mm})$ & $0.42 \times 0.30 \times 0.30$ \\
\hline \multicolumn{2}{|l|}{ Data collection } \\
\hline Diffractometer & Bruker AXS D8 Quest CMOS diffractometer \\
\hline Absorption correction & Multi-scan Apex2 v2014.11 (Bruker, 2014) \\
\hline$T_{\min }, T_{\max }$ & $0.295,0.536$ \\
\hline $\begin{array}{l}\text { No. of measured, independent and } \\
\text { observed }[I>2 \sigma(I)] \text { reflections }\end{array}$ & $93266,13050,11564$ \\
\hline$R_{\text {int }}$ & 0.039 \\
\hline$(\sin \theta / \lambda)_{\max }\left(\AA^{-1}\right)$ & 0.667 \\
\hline \multicolumn{2}{|l|}{ Refinement } \\
\hline$R\left[F^{2}>2 \sigma\left(F^{2}\right)\right], w R\left(F^{2}\right), S$ & $0.039,0.092,1.27$ \\
\hline No. of reflections & 13050 \\
\hline No. of parameters & 891 \\
\hline No. of restraints & 944 \\
\hline \multirow[t]{2}{*}{$\mathrm{H}$-atom treatment } & H-atom parameters constrained \\
\hline & $\begin{array}{l}w=1 /\left[\sigma^{2}\left(F_{0}^{2}\right)+122.3162 P\right] \\
\text { where } P=\left(F_{0}^{2}+2 F_{\mathrm{c}}^{2}\right) / 3\end{array}$ \\
\hline$\Delta \rho_{\max }, \Delta \rho_{\min }\left(\mathrm{e} \AA^{-3}\right)$ & $1.66,-1.71$ \\
\hline
\end{tabular}




\section{Refinement details:}

Disorder of a solvate THF molecule, of a 1,2,3,4,5-pentamethylcyclopentadienide ligand, and of one tolyl group are modeled in the structure. The tolyl group and the 1,2,3,4,5pentamethylcyclopentadienyl ligand were each refined as disordered over two positions. Geometries of the equivalent moieties were restrained to be similar, and $U^{i j}$ components of ADPs were restrained to be similar if closer than 1.7 Ångstrom. Subject to these conditions the occupancy ratios refined to $0.652(9)$ to $0.348(9)$ for the disordered tolyl group, and $0.600(7)$ and $0.400(7)$ for the pentamethyl cyclopentadienyl ligand.

A THF solvate molecule was refined as disordered over three overlapping positions. Geometries of the three moieties were again restrained to be similar, and Uij components of ADPs were restrained to be similar if closer than 1.7 Ångstrom. Subject to these conditions the occupancy rates, constrained to sum up to full occupancy, refined to $0.339(3), 0.467(3)$ and $0.193(3)$.

Reflection 202 was affected by the beam stop and has been omitted from the refinement. 


\section{Crystallographic Details $\left[\mathrm{Cp} * \mathrm{U}(\mathrm{NTol})_{2}\left({ }^{t} \mathrm{Bu}-\mathrm{MesPDI}^{\mathrm{Me}}\right)\right]\left[\mathrm{BPh}_{4}\right]$}

Local name: jk943

CCDC number: 1457422

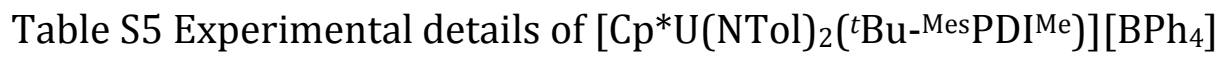

\begin{tabular}{|c|c|}
\hline \multicolumn{2}{|l|}{ Crystal data } \\
\hline Chemical formula & $2\left(\mathrm{C}_{55} \mathrm{H}_{68} \mathrm{~N}_{5} \mathrm{U}\right) \cdot 2\left(\mathrm{BC}_{24} \mathrm{H}_{20}\right) \cdot 3\left(\mathrm{C}_{4} \mathrm{H}_{8} \mathrm{O}\right)$ \\
\hline$M_{\mathrm{r}}$ & 2929.07 \\
\hline Crystal system, space group & Monoclinic, $P 2_{1} / c$ \\
\hline Temperature (K) & 100 \\
\hline$a, b, c(\AA)$ & $13.6883(2), 25.4867(6), 21.1460(3)$ \\
\hline$\beta\left(^{\circ}\right)$ & $101.7273(11)$ \\
\hline$V\left(\AA^{3}\right)$ & $7223.2(2)$ \\
\hline$Z$ & 2 \\
\hline Radiation type & $\mathrm{Cu} K \alpha$ \\
\hline$\mu\left(\mathrm{mm}^{-1}\right)$ & 6.70 \\
\hline Crystal size $(\mathrm{mm})$ & $0.20 \times 0.19 \times 0.10$ \\
\hline \multicolumn{2}{|l|}{ Data collection } \\
\hline Diffractometer & Rigaku Rapid II curved image plate diffractometer \\
\hline Absorption correction & Multi-scan SCALEPACK (Otwinowski \& Minor, 1997) \\
\hline$T_{\min }, T_{\max }$ & $0.310,0.554$ \\
\hline $\begin{array}{l}\text { No. of measured, independent and } \\
\text { observed }[I>2 \sigma(I)] \text { reflections }\end{array}$ & $105950,12844,11723$ \\
\hline$R_{\text {int }}$ & 0.071 \\
\hline$(\sin \theta / \lambda)_{\max }\left(\AA^{-1}\right)$ & 0.599 \\
\hline \multicolumn{2}{|l|}{ Refinement } \\
\hline$R\left[F^{2}>2 \sigma\left(F^{2}\right)\right], w R\left(F^{2}\right), S$ & $0.044,0.126,1.12$ \\
\hline No. of reflections & 12844 \\
\hline No. of parameters & 998 \\
\hline No. of restraints & 511 \\
\hline \multirow[t]{2}{*}{$\mathrm{H}$-atom treatment } & H-atom parameters constrained \\
\hline & $\begin{array}{l}w=1 /\left[\sigma^{2}\left(F_{0}^{2}\right)+(0.0796 P)^{2}+14.1131 P\right] \text { where } P= \\
\left(F_{0}^{2}+2 F_{\mathrm{c}}^{2}\right) / 3\end{array}$ \\
\hline$\Delta \rho_{\max }, \Delta \rho_{\min }\left(\mathrm{e} \AA^{-3}\right)$ & $2.46,-3.30$ \\
\hline
\end{tabular}




\section{Refinement details:}

A tert-butyl group is rotationally disordered over two positions. The two moieties were restrained to have similar geometries. Atoms were restrained to be close to isotropic and $\mathrm{U}^{\mathrm{ij}}$ components of ADPs were restrained to be similar if closer than 1.7 Ångstrom. The central C atoms of both moieties as well as C11 and C11B and C13 and C12B were constrained to have identical ADPs. Subject to these conditions the occupancy ratio refined to $0.640(8)$ to $0.360(8)$.

A THF molecule in a general position is disordered over two orientations. Another THF molecule located close to an inversion center is disordered with two alternative orientations that are in addition disordered via the center of inversion. $\mathrm{U}^{\mathrm{ij}}$ components of ADPs of THF molecules at both sites were restrained to be similar if closer than 1.7 Ångstrom. Subject to these conditions the occupancy ratios refined to 0.483(9) to 0.517(9) and $0.285(9)$ to $0.215(9)$. 


\section{Crystallographic Details 5-Cp*}

\section{Complex: [Cp*U(NPh $\left.)_{2}\left(\mathrm{MesPDI}^{\mathrm{Me}}\right)\right]\left[\mathrm{SbF}_{6}\right]$}

Local name: jk944

CCDC number: 1457423

\section{Table S6 Experimental details of 5-Cp*}

\begin{tabular}{|c|c|}
\hline \multicolumn{2}{|l|}{ Crystal data } \\
\hline Chemical formula & $4\left(\mathrm{C}_{49} \mathrm{H}_{56} \mathrm{~N}_{5} \mathrm{U}\right) \cdot 4\left(\mathrm{~F}_{6} \mathrm{Sb}\right) \cdot 4\left(\mathrm{C}_{4} \mathrm{H}_{8} \mathrm{O}\right) \cdot \mathrm{C}_{5} \mathrm{H}_{12}$ \\
\hline$M_{\mathrm{r}}$ & 5115.62 \\
\hline Crystal system, space group & Triclinic, $P \overline{1}$ \\
\hline Temperature (K) & 150 \\
\hline$a, b, c(\AA)$ & 16.3335 (7), $17.9889(9), 18.7027$ (10) \\
\hline$\alpha, \beta, \gamma\left({ }^{\circ}\right)$ & 96.147 (2), 96.853 (2), $102.820(2)$ \\
\hline$V\left(\AA^{3}\right)$ & $5269.1(4)$ \\
\hline$Z$ & 1 \\
\hline Radiation type & Mo $K \alpha$ \\
\hline$\mu\left(\mathrm{mm}^{-1}\right)$ & 3.65 \\
\hline Crystal size (mm) & $0.21 \times 0.20 \times 0.14$ \\
\hline \multicolumn{2}{|l|}{ Data collection } \\
\hline Diffractometer & Bruker AXS D8 Quest CMOS diffractometer \\
\hline Absorption correction & $\begin{array}{l}\text { Multi-scan } \\
\text { Apex2 v2014.11 (Bruker, 2014) }\end{array}$ \\
\hline$T_{\min }, T_{\max }$ & $0.616,0.746$ \\
\hline $\begin{array}{l}\text { No. of measured, independent and } \\
\text { observed }[I>2 \sigma(I)] \text { reflections }\end{array}$ & $163633,23204,19166$ \\
\hline$R_{\text {int }}$ & 0.036 \\
\hline$(\sin \theta / \lambda)_{\max }\left(\AA^{-1}\right)$ & 0.641 \\
\hline \multicolumn{2}{|l|}{ Refinement } \\
\hline$R\left[F^{2}>2 \sigma\left(F^{2}\right)\right], w R\left(F^{2}\right), S$ & $0.042,0.097,1.29$ \\
\hline No. of reflections & 23204 \\
\hline No. of parameters & 1354 \\
\hline No. of restraints & 265 \\
\hline \multirow[t]{2}{*}{$\mathrm{H}$-atom treatment } & $\mathrm{H}$-atom parameters constrained \\
\hline & $w=1 /\left[\sigma^{2}\left(F_{0}^{2}\right)+39.7784 P\right]$ where $P=\left(F_{0}^{2}+2 F_{c}^{2}\right) / 3$ \\
\hline$\Delta \rho_{\max }, \Delta \rho_{\min }\left(\mathrm{e} \AA^{-3}\right)$ & $3.72,-1.76$ \\
\hline
\end{tabular}




\section{Refinement details:}

A hexafluoroantimonate anion was refined as disordered over a major and minor moiety. The two units were restrained to have similar geometries, and the ADPs of the minor moiety fluorine atoms were constrained to be the same as their major moiety counterparts. Subject to these conditions the occupancy ratio refined to $0.881(6)$ to $0.119(6)$.

A THF molecule was refined as twofold disordered. The two units were restrained to have similar geometries as another not disordered THF molecule, and the Uij components of ADPs were restrained to be similar if closer than 1.7 Ångstrom. Subject to these conditions the occupancy ratio refined to $0.472(15)$ to $0.528(15)$.

An ill-defined pentane molecule is disordered around an inversion center. C-C bond distances were restrained to 1.45(2) Ångstrom, 1,3 distances to 2.5 Ångstrom. ADPs were restrained to be close to isotropic, and the atoms were subjected to a rigid bond restraint (RIGU in Shelxl). 
U L LIII-Edge XAFS Measurements. Samples of $\mathrm{Cp}^{\mathrm{P} U}(\mathrm{NPh})_{2}\left({ }^{\mathrm{Mes} P D I}{ }^{\mathrm{Me}}\right) \quad$ (2-Cp $\left.{ }^{\mathrm{P}}\right)$, $\mathrm{Cp} * \mathrm{U}(\mathrm{NPh})_{2}\left({ }^{\mathrm{Mes} P D I}{ }^{\mathrm{Me}}\right) \quad\left(\mathbf{2 - C p ^ { * } )}, \quad \mathrm{Cp} * \mathrm{U}(\mathrm{NTol})_{2}\left(\mathrm{MesPDI}^{\mathrm{Me}}\right) \quad\right.$ (3-Cp*), $\left[\mathrm{Cp}^{*} \mathrm{U}(\mathrm{NTol})_{2}\left(\mathrm{MesPDI}^{\mathrm{Me}}\right)\right]\left[\mathrm{SbF}_{6}\right]\left(\mathbf{4}-\mathrm{Cp}^{*}\right)$, and $\mathrm{Cp}^{*} \mathrm{UO}_{2}\left(\mathrm{MesPDI}^{\mathrm{Me}}\right)$ were analyzed by $\mathrm{U} \mathrm{L}_{3,2}$-edge $\mathrm{X}$-ray measurements. Samples were prepared under a nitrogen atmosphere. The samples were diluted with boron nitride (BN), which was dried at elevated temperature $\left(200{ }^{\circ} \mathrm{C}\right)$ under vacuum $\left(10^{-3}\right.$ Torr $)$ for $24 \mathrm{~h}$ prior to use. A mixture of the analyte and $\mathrm{BN}$ were weighed out, such that the edge jump for the absorbing atom was calculated to be at 1 absorption length in transmission ( $\sim 30$ to $50 \mathrm{mg}$ of sample and 50 to $30 \mathrm{mg}$ of BN). Samples were ground using a Wiggle Bug® using a Teflon bead and a polycarbontate capsule. The finely ground powders were pressed as a pellet into a slotted aluminum sample holder equipped with a Kapton windows (1 mil), one was fixed with super glue and the other was Kapton tape. This primary holder was nested within a secondary aluminum holder equipped with Kapton ( 2 mil) windows that were sealed with indium wire. The samples were sealed in ziplock bags, placed in sealed polypropylene jars, and shipped to SSRL. Once unpackaged, the samples were immediately attached to the coldfinger of a liquid $\mathrm{N}_{2}$ cryostat and quickly evacuated (10-7 Torr). The cryostat was attached to the beamline 11-2 XAFS rail (SSRL), which was equipped with three ionization chambers through which nitrogen gas was continually flowed. One chamber $(10 \mathrm{~cm})$ was positioned before the cryostat to monitor the incident radiation $\left(\mathrm{I}_{0}\right)$. The second chamber $(30 \mathrm{~cm})$ was positioned after the cryostat so that sample transmission $\left(\mathrm{I}_{1}\right)$ could be evaluated against $\mathrm{I}_{0}$ and so that the absorption coefficient $(\mu)$ could be calculated as $\ln \left(\mathrm{I}_{0} / \mathrm{I}_{1}\right)$. The third chamber $\left(\mathrm{I}_{2} ; 30 \mathrm{~cm}\right)$ was positioned downstream from $\mathrm{I}_{1}$ so that the XANES of a calibration foil could be measured against $\mathrm{I}_{1}$. A potential of $1600 \mathrm{~V}$ was applied in series to the ionization chambers. Samples were calibrated in-situ to the energy of the first inflection point of the K-edge of an yttrium foil (17038.4 eV).

The X-ray absorption near edge spectra (XANES) were measured at the Stanford Synchrotron Radiation Lightsource (SSRL), under dedicated operating conditions $(3.0 \mathrm{GeV}$, $5 \%, 500 \mathrm{~mA}$ using continuous topoff injections) on end station 11-2. This beamline, which was equipped with a 26-pole, 2.0 tesla wiggler, utilized a liquid nitrogen-cooled doublecrystal Si[220] monochromator and employed collimating and focusing mirrors. A single energy was selected from the white beam with a liquid- $\mathrm{N}_{2}$-cooled double-crystal monochromator utilizing Si[220] $(\varphi=0)$ crystals. Harmonic rejection was achieved by detuning the second crystal of the monochrometer by $35 \%$ at $17766 \mathrm{eV}$. The horizontal and vertical slit sizes were 10 and $1 \mathrm{~mm}$, respectively.

The data obtained could not be compared to our typical $\mathrm{Cs}_{2} \mathrm{UO}_{2} \mathrm{Cl}_{4}$ external standard because it was not measured under the same conditions as these compounds. Hence, we cannot directly compare these results with results previously obtained. However, all the samples reported here can be internally compared to each other, as the data were collected with an internal yttrium standard. 